\title{
Properties of distance spaces with power triangle inequalities
}

\author{
DANIEL J. GREENHOE
}

\begin{abstract}
Metric spaces provide a framework for analysis and have several very useful properties. Many of these properties follow in part from the triangle inequality. However, there are several applications in which the triangle inequality does not hold but in which we may still like to perform analysis. This paper investigates what happens if the triangle inequality is removed all together, leaving what is called a distance space, and also what happens if the triangle inequality is replaced with a much more general two parameter relation, which is herein called the "power triangle inequality". The power triangle inequality represents an uncountably large class of inequalities, and includes the triangle inequality, relaxed triangle inequality, and inframetric inequality as special cases. The power triangle inequality is defined in terms of a function that is herein called the power triangle function. The power triangle function is itself a power mean, and as such is continuous and monotone with respect to its exponential parameter, and also includes the operations of maximum, minimum, mean square, arithmetic mean, geometric mean, and harmonic mean as special cases.
\end{abstract}

2010 Mathematics Subject Classification 54E25 (primary); 54A05,54A20 (secondary)

Keywords: metric space, distance space, semimetric space, quasi-metric space, triangle inequality, relaxed triangle inequality, inframetric, arithmetic mean, means square, geometric mean, harmonic mean, maximum, minimum, power mean

\section{Contents}

1 Introduction and summary $r$

2 Standard definitions $\quad 4$

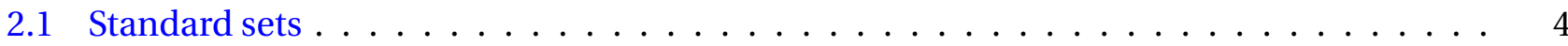

2.2 Relations ............................ 4

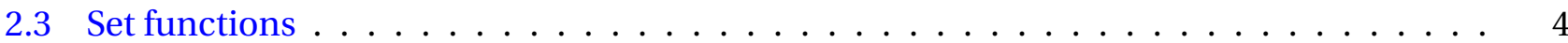

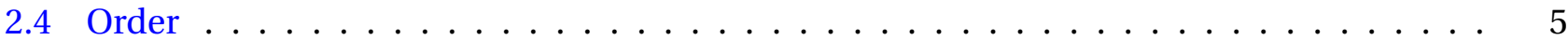

3 Background: distance spaces $\quad \mathbf{5}$

3.1 Fundamental structure of distance spaces . . . . . . . . . . . . . . . . . . . 6

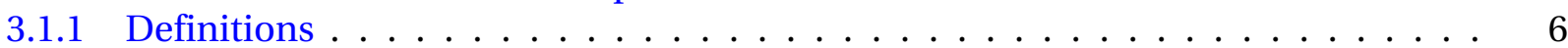

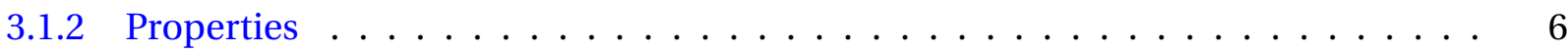

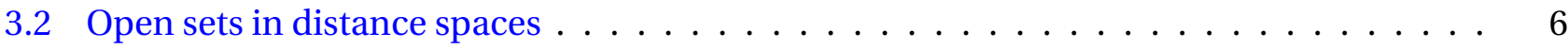

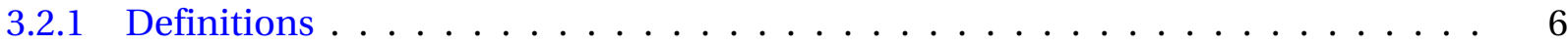

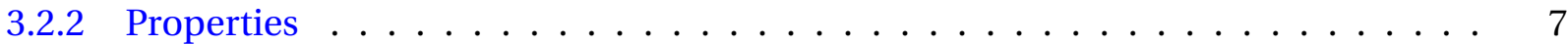

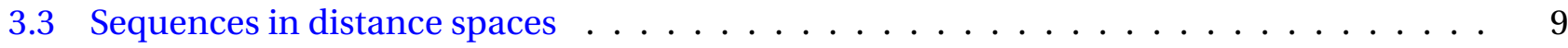

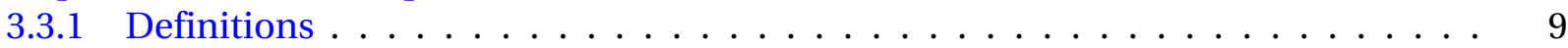

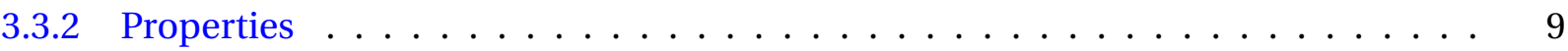

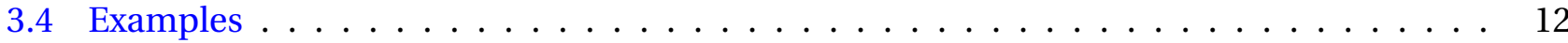


4 Distance spaces with power triangle inequalities 15

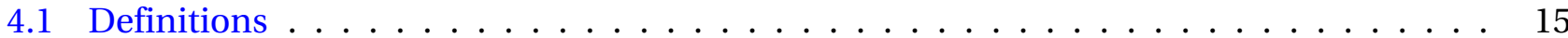

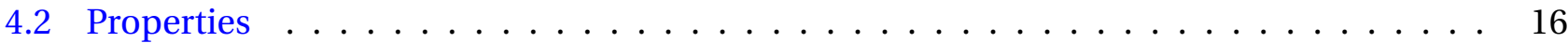

4.2.1 Relationships of the power triangle function $\ldots \ldots \ldots \ldots$

4.2 .2 Properties of power distance spaces . . . . . . . . . . . . . . 17

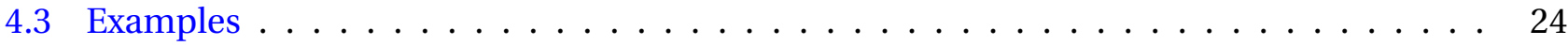

$\begin{array}{lr}\text { A Topological Spaces } & 26\end{array}$

B Finite sums $r$

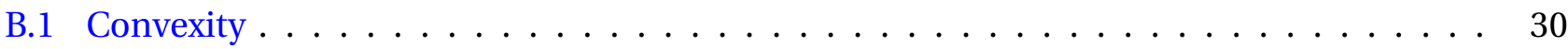

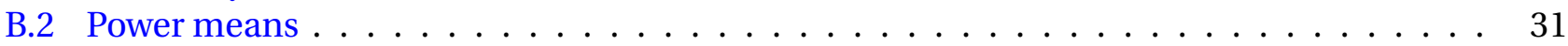

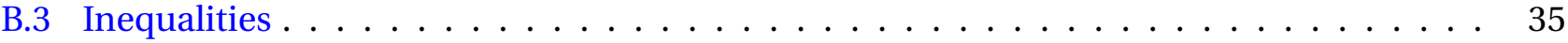

$\begin{array}{lr}\text { C Metric preserving functions } & 35\end{array}$

$\begin{array}{ll}\text { Bibliography } & 37\end{array}$

$\begin{array}{lr}\text { Reference Index } & 44\end{array}$

$\begin{array}{lr}\text { Subject Index } & 45\end{array}$

\section{Introduction and summary}

Metric spaces provide a framework for analysis and have several very useful properties. Many of these properties follow in part from the triangle inequality. However, there are several applications ${ }^{1}$ in which the triangle inequality does not hold but in which we would still like to perform analysis. So the questions that natually follow are:

Q1. What happens if we remove the triangle inequality all together?

Q2. What happens if we replace the triangle inequality with a generalized relation?

A distance space is a metric space without the triangle inequality constraint. Section 3 introduces distance spaces and demonstrates that some properties commonly associated with metric spaces also hold in any distance space:

$\begin{array}{lll}\text { D1. } & \varnothing \text { and } X \text { are open } & \text { (Theorem 3.7 page 7) } \\ \text { D2. } & \text { the intersection of a finite number of open sets is open } & \text { (Theorem 3.7 page 7) } \\ \text { D3. } & \text { the union of an arbitrary number of open sets is open } & \text { (Theorem 3.7 page 7) } \\ \text { D4. } & \text { every Cauchy sequence is bounded } & \text { (Proposition 3.14 page 9) } \\ \text { D5. } & \text { any subsequence of a Cauchy sequence is also Cauchy } & \text { (Proposition 3.15 page 10) } \\ \text { D6. } & \text { the Cantor Intersection Theorem holds } & \text { (Theorem 3.18 page 11) }\end{array}$

${ }^{1}$ references for applications in which the triangle inequality may not hold: 圈 [Maligranda and Orlicz(1987)] page $54\langle$ 〈pseudonorm"〉, 圈 [Lin(1998)]〈“similarity measures", Table 6〉, 圈 [Veltkamp and Hagedoorn(2000)] 〈"shape similarity measures"〉, 圈 [Veltkamp(2001)] “"shape matching”, 圈 [Costa et al.(2004)Costa, Castro, Rowstron, and Key] 〈"network distance estimation”〉, 圈 [Burstein et al.(2005)Burstein, Ulitsky, Tuller, and Chor] page 287 〈distance matrices for "genome phylogenies"〉, $\boxminus$ [Jiménez and Yukich(2006)] page 224 〈"statistical distances"〉, 圈 [Szirmai(2007)] page $388\langle$ “geodesic ball” $\rangle$, 圈 [Crammer et al.(2007)Crammer, Kearns, and Wortman] page 326 〈"decision-theoretic learning”〉, 圈 [Vitányi(2011)] page 2455 〈information distance”〉, 
The following five properties (M1-M5) do hold in any metric space. However, the examples from Section 3 listed below demonstrate that the five properties do not hold in all distance spaces:
M1. the metric function is continuous
fails to hold in Example 3.21-Example 3.23
M2. open balls are open
fails to hold in Example 3.21 and Example 3.22
M3. the open balls form a base for a topology
fails to hold in Example 3.21 and Example 3.22
M4. the limits of convergent sequences are unique
fails to hold in Example 3.21
M5. convergent sequences are Cauchy
fails to hold in Example 3.22

Hence, Section 3 answers question Q1.

Section 4 begins to answer question Q2 by first introducing a new function, called the power triangle function in a distance space $(X, \mathrm{~d})$, as $\tau(p, \sigma ; x, y, z ; \mathrm{d}) \triangleq 2 \sigma\left[\frac{1}{2} \mathrm{~d}^{p}(x, z)+\frac{1}{2} \mathrm{~d}^{p}(z, y)\right]^{\frac{1}{p}} \quad$ for some $(p, \sigma) \in$ $\mathbb{R}^{*} \times \mathbb{R} .^{2}$ Section 4 then goes on to use this function to define a new relation, called the power triangle inequality in $(X, \mathrm{~d})$, and defined as $\otimes(p, \sigma ; \mathrm{d}) \triangleq\left\{(x, y, z) \in X^{3} \mid \mathrm{d}(x, y) \leq \tau(p, \sigma ; x, y, z ; \mathrm{d})\right\}$.

The power triangle inequality is a generalized form of the triangle inequality in the sense that the two inequalities coincide at $(p, \sigma)=(1,1)$. Other special values include $(1, \sigma)$ yielding the relaxed triangle inequality (and its associated near metric space) and $(\infty, \sigma)$ yielding the $\sigma$-inframetric inequality (and its associated $\sigma$-inframetric space). Collectively, a distance space with a power triangle inequality is herein called a power distance space and denoted $(X, \mathrm{~d}, p, \sigma) .^{3}$

The power triangle function, at $\sigma=\frac{1}{2}$, is a special case of the power mean with $N=2$ and $\lambda_{1}=\lambda_{2}=$ $\frac{1}{2}$. Power means have the elegant properties of being continuous and monontone with respect to a free parameter $p$. From this it is easy to show that the power triangle function is also continuous and monontone with respect to both $p$ and $\sigma$. Special values of $p$ yield operators coinciding with maximum, minimum, mean square, arithmetic mean, geometric mean, and harmonic mean. Power means are briefly described in APPENDIX B.2. ${ }^{4}$

Section 4.2 investigates the properties of power distance spaces. In particular, it shows for what values of $(p, \sigma)$ the properties M1-M5 hold. Here is a summary of the results in a power distance space $(X, \mathrm{~d}, p, \sigma)$, for all $x, y, z \in X$ :
(M1) holds for any $(p, \sigma) \in\left(\mathbb{R}^{*} \backslash\{0\}\right) \times \mathbb{R}^{+}$such that $2 \sigma=2^{\frac{1}{p}}$
(M2) holds for any $(p, \sigma) \in\left(\mathbb{R}^{*} \backslash\{0\}\right) \times \mathbb{R}^{+}$such that $2 \sigma \leq 2^{\frac{1}{p}}$
(Theorem 4.18 page 23)
(M3) holds for any $(p, \sigma) \in\left(\mathbb{R}^{*} \backslash\{0\}\right) \times \mathbb{R}^{+}$such that $2 \sigma \leq 2^{\frac{1}{p}}$
(Corollary 4.14 page 21 )
(M4) holds for any $(p, \sigma) \in \mathbb{R}^{*} \times \mathbb{R}^{+}$
(Corollary 4.12 page 20)
(Theorem 4.19 page 23)
(M5) holds for any $(p, \sigma) \in \mathbb{R}^{*} \times \mathbb{R}^{+}$
(Theorem 4.16 page 21)

APPENDIX A briefly introduces topological spaces. The open balls of any metric space form a base for a topology. This is largely due to the fact that in a metric space, open balls are open. Because of this, in metric spaces it is convenient to use topological structure to define and exploit analytic concepts such as continuity, convergence, closed sets, closure, interior, and accumulation point. For example, in a metric space, the traditional definition of defining continuity using open balls and the topological

\footnotetext{
${ }^{2}$ where $\mathbb{R}^{*}$ is the set of extended real numbers and $\mathbb{R}^{+}$is the set of positive real numbers (Definition 2.1 page 4 )

${ }^{3}$ power triangle inequality: Definition 4.4 page 16; power distance space: Definition 4.3 page 16; examples of power distance space: Definition 4.5 page 16;

${ }^{4}$ power triangle function: Definition 4.1 (page 15); power mean: Definition B.6 (page 31); power mean is continuous and monontone: Theorem B.7 (page 31); power triangle function is continuous and monontone: Corollary 4.6 (page 16); Special values of $p$ : Corollary 4.7 (page 17), Corollary B.8 (page 34)
} 
definition using open sets, coincide with each other. Again, this is largely because the open balls of a metric space are open. ${ }^{5}$

However, this is not the case for all distance spaces. In general, the open balls of a distance space are not open, and they are not a base for a topology. In fact, the open balls of a distance space are a base for a topology if and only if the open balls are open. While the open sets in a distance space do induce a topology, it's open balls may not. ${ }^{6}$

\section{Standard definitions}

\subsection{Standard sets}

Definition 2.1 Let $\mathbb{R}$ be the set of real numbers. Let $\mathbb{R}^{\vdash} \triangleq\{x \in \mathbb{R} \mid x \geq 0\}$ be the set of non-negative real numbers. Let $\mathbb{R}^{+} \triangleq\{x \in \mathbb{R} \mid x>0\}$ be the set of postive real numbers. Let $\mathbb{R}^{*} \triangleq \mathbb{R} \cup\{-\infty, \infty\}$ be the set of extended real numbers. ${ }^{7}$ Let $\mathbb{Z}$ be the set of integers. Let $\mathbb{N} \triangleq\{n \in \mathbb{Z} \mid n \geq 1\}$ be the set of natural numbers. Let $\mathbb{Z}^{*} \triangleq \mathbb{Z} \cup\{-\infty, \infty\}$ be the extended set of integers.

Definition 2.2 Let $X$ be a set. The quantity $2^{X}$ is the power set of $X$ such that $2^{X} \triangleq\{A \subseteq X\} \quad$ (the set of all subsets of $X$ ).

\subsection{Relations}

Definition 2.3 ${ }^{8}$ Let $X$ and $Y$ be sets. The Cartesian product $X \times Y$ of $X$ and $Y$ is the set $X \times Y \triangleq$ $\{(x, y) \mid x \in X$ and $y \in Y\}$. An ordered pair $(x, y)$ on $X$ and $Y$ is any element in $X \times Y$. A relation ${ }^{\circledR}$ on $X$ and $Y$ is any subset of $X \times Y$ such that ${ }^{\circledR} \subseteq X \times Y$. The set $2^{X Y}$ is the set of all relations in $X \times Y$. A relation $\mathrm{f} \in \mathrm{2}^{X Y}$ is a function if $\left(x, y_{1}\right) \in \mathrm{f}$ and $\left(x, y_{2}\right) \in \mathrm{f} \Rightarrow y_{1}=y_{2}$. The set $Y^{X}$ is the set of all functions in $2^{X Y}$.

\subsection{Set functions}

Definition 2.4 ${ }^{9}$ Let $2^{X}$ be the power set (Definition 2.2 page 4) of a set $X$.

$$
\text { A set } S(X) \text { is a set structure on } X \text { if } \quad S(X) \subseteq 2^{X} \text {. }
$$

A set structure $\mathcal{Q}(X)$ is a paving on $X$ if $\varnothing \in \mathcal{Q}(X)$.

\footnotetext{
${ }^{5}$ open ball: Definition 3.5 page 6; metric space: Definition 4.5 page 16; base: Definition A.3 page 27; topology: Definition A.1 page 26; open: Definition 3.6 page 7; continuity in topological space: Definition A.11 page 28; convergence in distance space: Definition 3.11 page 9; convergence in topological space: Definition A.16 page 28; closed set: Definition A.1 page 26; closure, interior, accumulation point: Definition A.8 page 27; coincidence in all metric spaces and some power distance spaces: Theorem 4.15 page 21 ;

${ }^{6}$ if and only if statement: Theorem 3.10 page 8; open sets of a distance space induce a topology: Corollary 3.8 page 8;

${ }^{7} \boxminus[\operatorname{Rana}(2002)]$ pages 385-388 $\langle$ Appendix A〉

$8 \otimes[$ Maddux(2006)] page 4, $\boxminus$ [Halmos(1960)] pages 26-30, $\boxminus$ [Suppes(1972)] page 86, $\theta[\operatorname{Kelley}(1955)]$ page 10, $\theta$ [Bourbaki(1939)], $\boxminus\left[\right.$ Bottazzini(1986)] page 7, $\theta$ [Comtet(1974)] page $4\left\langle\left|Y^{X}\right|\right\rangle$; The notation $Y^{X}$ and $2^{X Y}$ is motivated by the fact that for finite $X$ and $Y,\left|Y^{X}\right|=|Y|^{|X|}$ and $\left|2^{X Y}\right|=2^{|X| \cdot|Y|}$.

${ }^{9} \boxminus[$ Molchanov(2005)] page 389, $\oslash[\operatorname{Pap}(1995)]$ page 7, $\bullet$ [Hahn and Rosenthal(1948)] page 254
} 
Definition 2.5 ${ }^{10}$ Let $\mathcal{Q}(X)$ be a paving (Definition 2.4 page 4) on a set $X$. Let $Y$ be a set containing the element 0 . A function $\mathrm{m} \in Y^{\mathcal{Q}(X)}$ is a set function if $\mathrm{m}(\varnothing)=0$.

Definition 2.6 The set function (Definition 2.5 page 5) $|A| \in \mathbb{Z}^{* \mathbb{Z}^{X}}$ is the cardinality of $A$ such that

$$
|A| \triangleq\left\{\begin{array}{ll}
\text { the number of elements in } A & \text { for finite } A \\
\infty & \text { otherwise }
\end{array}\right\} \quad \forall A \in 2^{X}
$$

Definition 2.7 Let $|X|$ be the cardinality (Definition 2.6 page 5) of a set $X$.

The structure $\varnothing$ is the empty set, and is a set such that $|\varnothing|=0$.

\subsection{Order}

Definition 2.8 ${ }^{11}$ Let $X$ be a set. A relation $\leq$ is an order relation in $2^{X X}$ (Definition 2.3 page 4) if

$\left.\begin{array}{llllll}\text { 1. } x \leq x & & & \forall x \in X & \text { (reflexive) } & \text { and } \\ \text { 2. } x \leq y \text { and } y \leq z \Longrightarrow x \leq z & \forall x, y \in X & \text { (transitive) } & \text { and }\end{array}\right]$ preorder

An ordered set is the pair $(X, \leq)$.

Definition 2.9 ${ }^{12}$ In an ordered set $(X, \leq)$,

the set $[x: y] \triangleq\{z \in X \mid x \leq z \leq y\}$ is a closed interval and

the set $(x: y] \triangleq\{z \in X \mid x<z \leq y\}$ is a half-open interval and

the set $[x: y) \triangleq\{z \in X \mid x \leq z<y\}$ is a half-open interval and

the set $(x: y) \triangleq\{z \in X \mid x<z<y\}$ is an open interval.

Definition 2.10 Let $(\mathbb{R}, \leq)$ be the ordered set of real numbers (Definition 2.8 page 5).

The absolute value $|\cdot| \in \mathbb{R}^{\mathbb{R}}$ is defined as ${ }^{13} \quad|x| \triangleq\left\{\begin{aligned}-x & \text { for } x \leq 0 \\ x & \text { otherwise }\end{aligned}\right\}$.

\section{Background: distance spaces}

A distance space (Definition 3.1 page 6) can be defined as a metric space (Definition 4.5 page 16) without the triangle inequality constraint. Much of the material in this section about distance spaces is standard in metric spaces. However, this paper works through this material again to demonstrate "how far we can go", and can't go, without the triangle inequality.

\footnotetext{
${ }^{10} \boxminus$ [Hahn and Rosenthal(1948)], 圈 [Choquet(1954)], $\theta[\operatorname{Pap}(1995)]$ page 8 〈Definition 2.3: extended realvalued set function $\rangle, \otimes[\operatorname{Halmos}(1950)]$ page $30\langle \$ 7$. MEASURE ON RINGS $\rangle$,

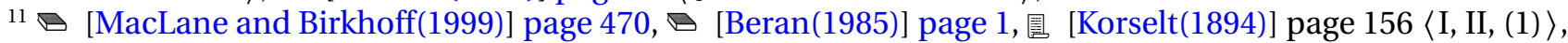
圈 [Dedekind(1900)] page $373\langle\mathrm{I}-\mathrm{III}\rangle$. An order relation is also called a partial order relation. An ordered set is also called a partially ordered set or poset.

$12 \ominus[$ Apostol(1975)] page $4, \otimes[$ Ore(1935)] page 409

${ }^{13}$ A more general definition for absolute value is available for any commutative ring: Let $R$ be a commutative ring. A function $|\cdot|$ in $R^{R}$ is an absolute value, or modulus, on $R$ if

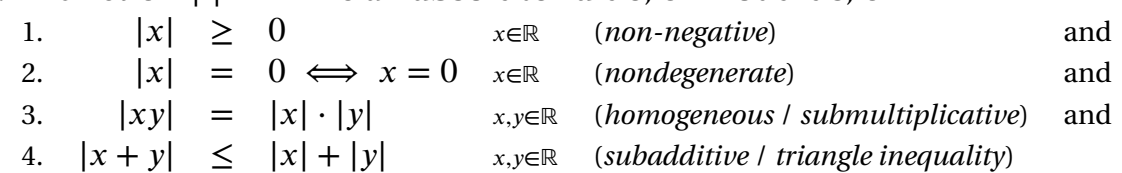

Reference: $\nabla$ [Cohn(2002 December 6)] page 312
} 


\subsection{Fundamental structure of distance spaces}

\subsubsection{Definitions}

Definition 3.1 14 A function $d$ in the set $\mathbb{R}^{X \times X}$ (Definition 2.3 page 4) is a distance if

$\begin{array}{llllll}\text { 1. } & \mathrm{d}(x, y) \geq 0 & & \forall x, y \in X & \text { (non-negative) } & \text { and } \\ \text { 2. } \mathrm{d}(x, y) & =0 \Longleftrightarrow x=y & \forall x, y \in X & \text { (nondegenerate) } & \text { and } \\ \text { 3. } \mathrm{d}(x, y)=\mathrm{d}(y, x) & \forall x, y \in X & \text { (symmetric) } & \end{array}$

The pair $(X, \mathrm{~d})$ is a distance space if $\mathrm{d}$ is a distance on a set $X$.

Definition 3.2 ${ }^{15}$ Let $(X, \mathrm{~d})$ be a distance space and $2^{X}$ be the power set of $X$ (Definition 2.2 page 4). The diameter in $(X, \mathrm{~d})$ of a set $A \in 2^{X}$ is $\quad \operatorname{diam} A \triangleq \begin{cases}0 & \text { for } A=\varnothing \\ \sup \{\mathrm{d}(x, y) \mid x, y \in A\} & \text { otherwise }\end{cases}$

Definition 3.3 ${ }^{16}$ Let $(X, \mathrm{~d})$ be a distance space. Let $2^{X}$ be the power set (Definition 2.2 page 4) of $X$. A set $A$ is bounded in $(X, \mathrm{~d})$ if $A \in 2^{X}$ and $\operatorname{diam} A<\infty$.

\subsubsection{Properties}

Remark 3.4 Let $\left(x_{n}\right)_{n \in \mathbb{Z}}$ be a sequence in a distance space $(X, \mathrm{~d})$. The distance space $(X, \mathrm{~d})$ does not necessarily have all the nice properties that a metric space (Definition 4.5 page 16$)$ has. In particular, note the following:

1. $\mathrm{d}$ is a distance in $(X, \mathrm{~d})$

$\Longrightarrow \quad \mathrm{d}$ is continuous in $(X, \mathrm{~d})$

(Example 3.23 page 14).

2. $\mathrm{B}$ is an open ball in $(X, \mathrm{~d})$

$\Rightarrow \quad \mathrm{B}$ is open in $(X, \mathrm{~d})$

(Example 3.22 page 13).

3. $\boldsymbol{B}$ is the set of all open balls in $(X, \mathrm{~d})$

$\Rightarrow \quad B$ is a base for a topology on $X$

(Example 3.22 page 13). ${ }^{17}$

4. $\left(x_{n}\right)$ is convergent in $(X, \mathrm{~d})$

$\Longrightarrow \quad$ limit is unique

(Example 3.21 page 12).

5. $\left(x_{n}\right)$ is convergent in $(X, \mathrm{~d})$

$\Longrightarrow \quad\left(x_{n}\right)$ is Cauchy in $(X, \mathrm{~d})$

(Example 3.22 page 13).

\subsection{Open sets in distance spaces}

\subsubsection{Definitions}

Definition 3.5 ${ }^{18}$ Let $(X, \mathrm{~d})$ be a distance space (Definition 3.1 page 6$)$. Let $\mathbb{R}^{+}$be the set of positive real numbers (Definition 2.1 page 4).

An open ball centered at $x$ with radius $r$ is the set $\mathrm{B}(x, r) \triangleq\{y \in X \mid \mathrm{d}(x, y)<r\}$.

A closed ball centered at $x$ with radius $r$ is the set $\overline{\mathrm{B}}(x, r) \triangleq\{y \in X \mid \mathrm{d}(x, y) \leq r\}$.

${ }^{14}$ 圈 [Menger(1928)] page 76 〈"Abstand $a b$ definiert ist..." (distance from $a$ to $b$ is defined as...") 〉, 圈 [Wilson(1931)] page 361〈§1., "distance", "semi-metric space" 〉, 图 [Blumenthal(1938)] page 38, $\theta$ [Blumenthal(1953)] page 7 〈"Definition 5.1. A distance space is called semimetric provided..."〉, 圈 [Galvin and

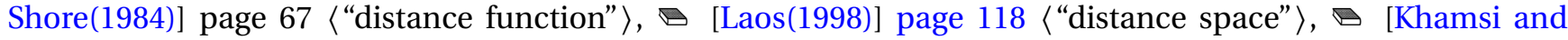
Kirk(2001)] page $13\langle$ "semimetric space"〉, 圈 [Bessenyei and Pales(2014)] page $2\langle$ "semimetric space" $\rangle, \theta$ [Deza and Deza(2014)] page 3 〈"distance (or dissimilarity)"〉

${ }^{15}$ in metric space: $\oslash$ [Hausdorff(1937)], page 166, $\bullet[$ Copson(1968)], page 23, $\oslash$ [Michel and Herget(1993)], page 267, $\boxminus$ [Molchanov(2005)] page 389

${ }^{16}$ in metric space: $\boxminus$ [Thron(1966)], page 154 〈definition 19.5〉, $\boxminus$ [Bruckner et al.(1997)Bruckner, Bruckner, and Thomson] page 356

${ }^{17}$ 圈 [Heath(1961)] page 810 〈THEOREM〉, 圈 [Galvin and Shore(1984)] page 71 〈2.3 LEMMA〉

${ }^{18}$ in metric space: $\because$ [Aliprantis and Burkinshaw(1998)], page 35 
Definition 3.6 Let $(X, \mathrm{~d})$ be a distance space. Let $X \backslash A$ be the set difference of $X$ and a set $A$. A set $U$ is open in $(X, \mathrm{~d})$ if $U \in 2^{X}$ and for every $x$ in $U$ there exists $r \in \mathbb{R}^{+}$such that $\mathrm{B}(x, r) \subseteq U$. A set $U$ is an open set in $(X, \mathrm{~d})$ if $U$ is open in $(X, \mathrm{~d})$. A set $D$ is closed in $(X, \mathrm{~d})$ if $(X \backslash D)$ is open. A set $D$ is a closed set in $(X, d)$ if $D$ is closed in $(X, d)$.

\subsubsection{Properties}

Theorem $3.7{ }^{19}$ Let $(X, \mathrm{~d})$ be a DISTANCE SPACE. Let $N$ be any (finite) positive integer. Let $\Gamma$ be a SET possibly with an uncountable number of elements.

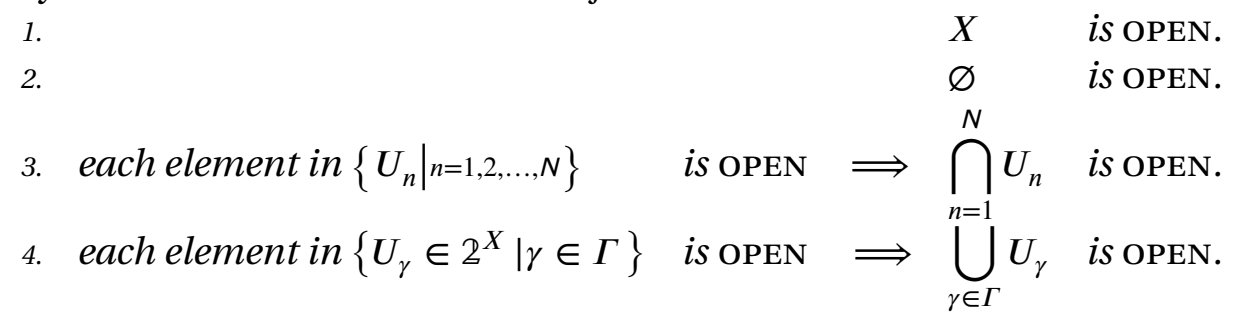

QPRoof:

(1) Proof that $X$ is open in $(X, d)$ :

(a) By definition of open set (Definition 3.6 page 7), $X$ is open $\Longleftrightarrow \forall x \in X \quad \exists r$ such that $\quad \mathrm{B}(x, r) \subseteq X$.

(b) By definition of open ball (Definition 3.5 page 6), it is always true that $\mathrm{B}(x, r) \subseteq X$ in $(X, \mathrm{~d})$.

(c) Therefore, $X$ is open in $(X, \mathrm{~d})$.

(2) Proof that $\varnothing$ is open in $(X, d)$ :

(a) By definition of open set (Definition 3.6 page 7), $\varnothing$ is open $\Longleftrightarrow \forall x \in X \quad \exists r$ such that $\mathrm{B}(x, r) \subseteq \varnothing$.

(b) By definition of empty set $\varnothing$ (Definition 2.7 page 5), this is always true because no $x$ is in $\varnothing$.

(c) Therefore, $\varnothing$ is open in $(X, d)$.

(3) Proof that $\bigcup U_{\gamma}$ is open in $(X, d)$ :

(a) By definition of open set (Definition 3.6 page 7), $\bigcup U_{\gamma}$ is open $\Longleftrightarrow \forall x \in \bigcup U_{\gamma} \quad \exists r$ such that $\mathrm{B}(x, r) \subseteq \bigcup U_{\gamma}$.

(b) If $x \in \bigcup U_{\gamma}$, then there is at least one $U \in \bigcup U_{\gamma}$ that contains $x$.

(c) By the left hypothesis in (4), that set $U$ is open and so for that $x, \exists r$ such that $\mathrm{B}(x, r) \subseteq U \subseteq \cup U_{\gamma}$.

(d) Therefore, $\bigcup U_{\gamma}$ is open in $(X, \mathrm{~d})$.

(4) Proof that $U_{1}$ and $U_{2}$ are open $\Longrightarrow U_{1} \cap U_{2}$ is open:

(a) By definition of open set (Definition 3.6 page 7), $U_{1} \cap U_{2}$ is open $\Longleftrightarrow \forall x \in U_{1} \cap U_{2} \quad \exists r$ such that $\mathrm{B}(x, r) \subseteq$ $U_{1} \cap U_{2}$.

(b) By the left hypothesis above, $U_{1}$ and $U_{2}$ are open; and by the definition of open sets (Definition 3.6 page 7), there exists $r_{1}$ and $r_{2}$ such that $\mathrm{B}\left(x, r_{1}\right) \subseteq U_{1}$ and $\mathrm{B}\left(x, r_{2}\right) \subseteq U_{2}$.

(c) Let $r \triangleq \min \left\{r_{1}, r_{2}\right\}$. Then $\mathrm{B}(x, r) \subseteq U_{1}$ and $\mathrm{B}(x, r) \subseteq U_{2}$.

(d) By definition of set intersection $\cap$ then, $\mathrm{B}(x, r) \subseteq U_{1} \cap U_{2}$.

(e) By definition of open set (Definition 3.6 page 7), $U_{1} \cap U_{2}$ is open.

(5) Proof that $\bigcap_{n=1}^{N} U_{n}$ is open (by induction):

(a) Proof for $N=1$ case: $\bigcap_{n=1}^{N} U_{n}=\bigcap_{n=1}^{1} U_{n}=U_{1}$ is open by hypothesis.

\footnotetext{
${ }^{19}$ in metric space: $\bullet$ [Dieudonné(1969)], pages 33-34, $\bullet \quad[$ Rosenlicht(1968)] page 39
} 
(b) Proof that $N$ case $\Longrightarrow N+1$ case:

$$
\begin{aligned}
\bigcap_{n=1}^{N+1} U_{n} & =\left(\bigcap_{n=1}^{N} U_{n}\right) \cap U_{N+1} & & \text { by property of } \bigcap \\
& \Longrightarrow \text { open } & & \text { by “ } N \text { case” hypothesis and (4) lemma page } 7
\end{aligned}
$$

Corollary 3.8 Let $(X, \mathrm{~d})$ be a Distance SPaCe. The set $T \triangleq\left\{U \in 2^{X} \mid U\right.$ is open in $\left.(X, \mathrm{~d})\right\}$ is a TOPOLOGY on $X$, and $(X, T)$ is $a$ TOPOLOGOGICAL SPACE.

QProof: This follows directly from the definition of an open set (Definition 3.6 page 7), Theorem 3.7 (page 7), and the definition of topology (Definition A.1 page 26).

Of course it is possible to define a very large number of topologies even on a finite set with just a handful of elements; ${ }^{20}$ and it is possible to define an infinite number of topologies even on a linearly ordered infinite set like the real line $(\mathbb{R}, \leq) .{ }^{21}$ Be that as it may, Definition 3.9 (next definition) defines a single but convenient topological space in terms of a distance space. Note that every metric space conveniently and naturally induces a topological space because the open balls of the metric space form a base for the topology. This is not the case for all distance spaces. But if the open balls of a distance space are all open, then those open balls induce a topology (next theorem).$^{22}$

Definition 3.9 Let $(X, \mathrm{~d})$ be a distance space. The set $T \triangleq\left\{U \in 2^{X} \mid U\right.$ is open in $\left.(X, \mathrm{~d})\right\}$ is the topology induced by $(X, \mathrm{~d})$ on $X$. The pair $(X, \boldsymbol{T})$ is called the topological space induced by $(X, \mathrm{~d})$.

For any distance space $(X, \mathrm{~d})$, no matter how strange, there is guaranteed to be at least one topological space induced by $(X, \mathrm{~d})$-and that is the indiscrete topological space (Example A.2 page 26) because for any distance space $(X, \mathrm{~d}), \varnothing$ and $X$ are open sets in $(X, \mathrm{~d})$ (Theorem 3.7 page 7 ).

Theorem 3.10 Let $\boldsymbol{B}$ be the set of all OPEN BALLs in a DISTANCE SPACE $(X, \mathrm{~d})$. \{every OPEN BALL in $\boldsymbol{B}$ is OPEN $\} \quad \Longleftrightarrow \quad\{\boldsymbol{B}$ is $a$ BASE for $a$ TOPOLOGY $\}$

\footnotetext{
${ }^{20}$ For a finite set $X$ with $n$ elements, there are 29 topologies on $X$ if $n=3,6942$ topologies on $X$ if $n=5$, and and 8,977,053,873,043 (almost 9 trillion) topologies on $X$ if $n=10$. References: 모. [oei(2014)] 〈http:// oeis.org/A000798 , $\boxminus$ [Brown and Watson(1996)], page 31, $\boxminus$ [Comtet(1974)] page 229, $\boxminus$ [Comtet(1966)], $\otimes[$ Chatterji(1967)], page 7, $\bullet$ [Evans et al.(1967)Evans, Harary, and Lynn], $\bullet$ [Krishnamurthy(1966)], page 157

${ }^{21}$ For examples of topologies on the real line, see the following: $\theta$ [Adams and Franzosa(2008)] page 31 〈"six topologies on the real line" $\rangle, \otimes$ [Salzmann et al.(2007)Salzmann, Grundhöfer, Hähl, and Löwen] pages 64-70 $\langle$ Weird topologies on the real line $\rangle, \boxminus[$ Murdeshwar(1990)] page $53\langle$ "often used topologies on the real line" , $\forall$ [Joshi(1983)] pages 85-91 〈\$4.2 Examples of Topological Spaces〉

${ }^{22}$ metric space: Definition 4.5 page 16; open ball: Definition 3.5 page 6; base: Definition A.3 page 27; topology: Definition A.1 page 26; not all open balls are open in a distance space: Example 3.21 (page 12) and Example 3.22 (page 13);
} 
ProOF:

every open ball in $\boldsymbol{B}$ is open

$\Longrightarrow$ for every $x$ in $B_{y} \in \boldsymbol{B}$ there exists $r \in \mathbb{R}^{+}$such that $\mathrm{B}(x, r) \subseteq B_{y} \quad$ by definition of open (Definition 3.6 page 7)

$\Longrightarrow\left\{\begin{array}{l}\text { for every } x \in X \text { and for every } B_{y} \in \boldsymbol{B} \text { containing } x, \\ \text { there exists } B_{x} \in \boldsymbol{B} \text { such that } \quad x \in B_{x} \subseteq B_{y} .\end{array}\right\} \quad$ because $\forall(x, r) \in X \times \mathbb{R}^{+}, \mathrm{B}(x, r) \subseteq X$

$\Longrightarrow B$ is a base for $T$

$\Longrightarrow\left\{\begin{array}{l}\text { for every } x \in X \text { and for every } U \subseteq T \text { containing } x, \\ \text { there exists } B_{x} \in \boldsymbol{B} \text { such that } \quad x \in B_{x} \subseteq U .\end{array}\right\} \quad$ by Theorem A.4 page 27

$\Longrightarrow\left\{\begin{array}{l}\text { for every } x \in X \text { and for every } B_{y} \in \boldsymbol{B} \subseteq \boldsymbol{T} \text { containing } x, \\ \text { there exists } B_{x} \in \boldsymbol{B} \text { such that } \quad x \in B_{x} \subseteq B_{y} .\end{array}\right\} \quad$ by definition of base (Definition A.3 page 27)

$\Longrightarrow\left\{\begin{array}{l}\text { for every } x \in B_{y} \in \boldsymbol{B} \subseteq \boldsymbol{T}, \\ \text { there exists } B_{x} \in \boldsymbol{B} \text { such that } \quad x \in B_{x} \subseteq B_{y} .\end{array}\right\}$

$\Longrightarrow$ every open ball in $\boldsymbol{B}$ is open

by definition of open (Definition 3.6 page 7)

\subsection{Sequences in distance spaces}

\subsubsection{Definitions}

Definition 3.1 ${ }^{23}$ Let $\left(x_{n} \in X\right)_{n \in \mathbb{Z}}$ be a sequence in a distance space $(X, \mathrm{~d})$. The sequence $\left(x_{n}\right)$ converges to a limit $x$ if for any $\varepsilon \in \mathbb{R}^{+}$, there exists $N \in \mathbb{Z}$ such that for all $n>N, \mathrm{~d}\left(x_{n}, x\right)<\varepsilon$.

This condition can be expressed in any of the following forms:

1. The limit of the sequence $\left(x_{n}\right)$ is $x . \quad 3 . \lim _{n \rightarrow \infty}\left(x_{n}\right)=x$.

2. The sequence $\left(x_{n}\right)$ is convergent with limit $x . \quad$ 4. $\left(x_{n}\right) \rightarrow x$.

A sequence that converges is convergent.

Definition 3.12 ${ }^{24}$ Let $\left(x_{n} \in X\right)_{n \in \mathbb{Z}}$ be a sequence in a distance space $(X, \mathrm{~d})$.

The sequence $\left(x_{n}\right)$ is a Cauchy sequence in $(X, \mathrm{~d})$ if

for every $\varepsilon \in \mathbb{R}^{+}$, there exists $N \in \mathbb{Z}$ such that $\forall n, m>N, \mathrm{~d}\left(x_{n}, x_{m}\right)<\varepsilon \quad$ (Cauchycondition).

Definition 3.13 ${ }^{25}$ Let $\left(x_{n} \in X\right)_{n \in \mathbb{Z}}$ be a sequence in a distance space $(X, \mathrm{~d})$.

The sequence $\left(x_{n} \in X\right)_{n \in \mathbb{Z}}$ is complete in $(X, \mathrm{~d})$ if

$\left(x_{n}\right)$ is Cauchy in $(X, \mathrm{~d}) \quad \Longrightarrow \quad\left(x_{n}\right)$ is convergent in $(X, \mathrm{~d})$.

\subsubsection{Properties}

Proposition 3.14 ${ }^{26}$ Let $\left(x_{n} \in X\right)_{n \in \mathbb{Z}}$ be a SEQuenCe in a Distance SPACE $(X, \mathrm{~d})$. $\left\{\left(x_{n}\right) \text { is CAuchy in }(X, \mathrm{~d})\right\}^{2} \Longrightarrow \quad\left\{\left(x_{n}\right)\right.$ is BOUnded in $\left.(X, \mathrm{~d})\right\}$

${ }^{23}$ in metric space: $\otimes$ [Rosenlicht(1968)] page 45, $\theta$ [Giles(1987)] page $37\langle 3.2$ Definition $\rangle, \theta$ [Khamsi and Kirk(2001)] page $13\langle$ Definition 2.1〉 “ $\rightarrow$ ” symbol: $\theta$ [Leathem(1905)] page 13 〈section III.11〉

${ }^{24}$ in metric space: $\boxminus$ [Apostol(1975)] page $73\langle 4.7\rangle, \otimes$ [Rosenlicht(1968)] page 51

${ }^{25}$ in metric space: $\boxminus$ [Rosenlicht(1968)] page 52

${ }^{26}$ in metric space: $\otimes$ [Giles(1987)] page $49\langle$ Theorem 3.30〉 
QRoof:

$\left(x_{n}\right)$ is Cauchy $\Longrightarrow$ for every $\varepsilon \in \mathbb{R}^{+}, \exists N \in \mathbb{Z} \quad$ such that $\forall n, m>N, \mathrm{~d}\left(x_{n}, x_{m}\right)<\varepsilon \quad$ (by Definition 3.12 page 9)

$\Longrightarrow \exists N \in \mathbb{Z}$ such that $\forall n, m>N, \mathrm{~d}\left(x_{n}, x_{m}\right)<1 \quad$ (arbitrarily choose $\varepsilon \triangleq 1$ )

$\Longrightarrow \exists N \in \mathbb{Z}$ such that $\forall n, m \in \mathbb{Z}, \mathrm{d}\left(x_{n}, x_{m+1}\right)<\max \left\{\{1\} \cup\left\{\mathrm{d}\left(x_{p}, x_{q}\right) \mid p, q \ngtr N\right\}\right\}$

$\Longrightarrow\left(x_{n}\right)$ is bounded (by Definition 3.3 page 6)

Proposition 3.15 ${ }^{27}$ Let $\left(x_{n} \in X\right)_{n \in \mathbb{Z}}$ be a SEQUENCE in a DISTANCE SPACE $(X, \mathrm{~d})$. Let $\mathrm{f} \in \mathbb{Z}^{\mathbb{Z}}$ (Definition 2.3 page 4) be a STRICTLY MONOTONE function such that $\mathrm{f}(n)<\mathrm{f}(n+1)$.

$$
\underbrace{\left(\left(x_{n}\right)_{n \in \mathbb{Z}} \text { is } \mathrm{CAUCHY}\right.}_{\text {sequence is } \mathrm{CAUCHY}} \Longrightarrow \underbrace{\left(x_{\mathrm{f}(n)}\right)_{n \in \mathbb{Z}} \text { is } \mathrm{CAUCHY}}_{\text {subsequence is also } \mathrm{CAUCHY}}
$$

QPRoof:

$$
\begin{aligned}
& \left(x_{n}\right)_{n \in \mathbb{Z}} \text { is Cauchy } \\
& \Longrightarrow \text { for any given } \varepsilon>0, \exists N \text { such that } \forall n, m>N, \mathrm{~d}\left(x_{n}, x_{m}\right)<\varepsilon \\
& \Longrightarrow \text { for any given } \varepsilon>0, \exists N^{\prime} \text { such that } \forall \mathrm{f}(n), \mathrm{f}(m)>N^{\prime}, \mathrm{d}\left(x_{\mathrm{f}(n)}, x_{\mathrm{f}(m)}\right)<\varepsilon \\
& \Longrightarrow\left(x_{\mathrm{f}(n)}\right)_{n \in \mathbb{Z}} \text { is Cauchy }
\end{aligned}
$$

by Definition 3.12 page 9

by Definition 3.12 page 9

Theorem 3.16 ${ }^{28}$ Let $(X, \mathrm{~d})$ be a DISTANCE SPACE. Let $A^{-}$be the ClOSURE (Definition A.8 page 27) of a $A$ in $a$ TOPOLOGICAL SPACE INDUCED BY $(X, \mathrm{~d})$.

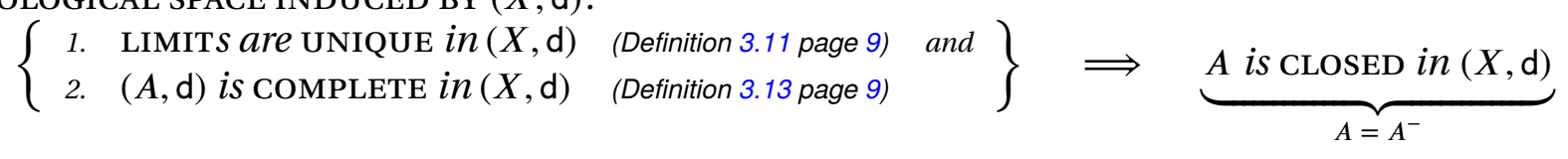

QPROOF:

(1) Proof that $A \subseteq A^{-}$: by Lemma A.10 page 27

(2) Proof that $A^{-} \subseteq A$ (proof that $x \in A^{-} \Longrightarrow x \in A$ ):

(a) Let $x$ be a point in $A^{-}\left(x \in A^{-}\right)$.

(b) Define a sequence of open balls $\left(\mathrm{B}\left(x, \frac{1}{1}\right), \mathrm{B}\left(x, \frac{1}{2}\right), \mathrm{B}\left(x, \frac{1}{3}\right), \ldots\right)$.

(c) Define a sequence of points $\left(x_{1}, x_{2}, x_{3}, \ldots\right)$ such that $x_{n} \in \mathrm{B}\left(x_{n}, \frac{1}{n}\right) \cap A$.

(d) Then $\left(x_{n}\right)$ is convergent in $X$ with limit $x$ by Definition 3.11 page 9

(e) and $\left(x_{n}\right)$ is Cauchy in $A$ by Definition 3.12 page 9 .

(f) By the hypothesis $2,\left(x_{n}\right)$ is therefore also convergent in $A$. Let this limit be $y$. Note that $y \in A$.

(g) By hypothesis 1 , limits are unique, so $y=x$.

(h) Because $y \in A$ (item (2f)) and $y=x$ (item (2g)), so $x \in A$.

(i) Therefore, $x \in A^{-} \Longrightarrow x \in A$ and $A^{-} \subseteq A$.

Proposition 3.17 ${ }^{29}$ Let $\left(x_{n}\right)_{n \in \mathbb{Z}}$ be a sequence in a DISTANCE SPACE $(X, \mathrm{~d})$. Let $\mathrm{f}: \mathbb{Z} \rightarrow \mathbb{Z}$ be a strictly

\footnotetext{
${ }^{27}$ in metric space: $\theta$ [Rosenlicht(1968)] page 52

${ }^{28}$ in metric space: $₫$ [Kubrusly(2001)] page $128\langle$ Theorem 3.40〉, $\boxminus$ [Haaser and Sullivan(1991)] page 75 $\langle 6 \cdot 10,6 \cdot 11$ Propositions $\rangle, \boxminus[$ Bryant(1985)] page $40\langle$ Theorem 3.6, 3.7,$\otimes$ [Sutherland(1975)] pages $123-124$

${ }^{29}$ in metric space: $\boxminus$ [Rosenlicht(1968)] page 46
} 
increasing function such that $\mathrm{f}(n)<\mathrm{f}(n+1)$.

$$
\underbrace{\left(x_{n}\right)_{n \in \mathbb{Z}} \rightarrow x}_{\text {converges to limit } x} \Longrightarrow \underbrace{\left(x_{\mathrm{f}(n)}\right)_{n \in \mathbb{Z}} \rightarrow x}_{\text {subsequence converges to the same limit } x}
$$

QRoof:

$$
\begin{aligned}
\left(x_{n}\right)_{n \in \mathbb{Z}} \rightarrow x & \Longrightarrow \forall \varepsilon>0, \exists N \quad \text { such that } \forall n>N, \mathrm{~d}\left(x_{n}, x\right)<\varepsilon \\
& \Longrightarrow \forall \varepsilon>0, \exists \mathrm{f}(N) \text { such that } \forall \mathrm{f}(n)>\mathrm{f}(N), \mathrm{d}\left(x_{\mathrm{f}(n)}, x\right)<\varepsilon \\
& \Longrightarrow\left(x_{\mathrm{f}(n)}\right)_{n \in \mathbb{Z}} \rightarrow x
\end{aligned}
$$

by Theorem 4.15 page 21

by Theorem 4.15 page 21

Theorem 3.18 (Cantor intersection theorem) 30 Let $(X, \mathrm{~d})$ be a DISTANCE SPACE (Definition 3.1 page 6), $\left(A_{n}\right)_{n \in \mathbb{Z}}$ a SEQUENCE with each $A_{n} \in 2^{X}$, and $|A|$ the number of elements in $A$.

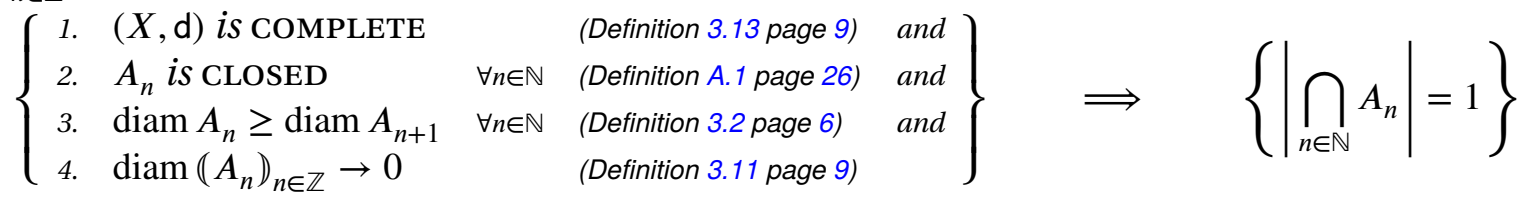

Proof:

(1) Proof that $\left|\bigcap_{n \in \mathbb{Z}} A_{n}\right|<2$ :
(a) Let $A \triangleq \cap A_{n}$.
(b) $x \neq y$ and $\{x, y\} \in A \Longrightarrow \mathrm{d}(x, y)>0$ and $\{x, y\} \subseteq A_{n} \forall n$
(c) $\exists n$ such that $\operatorname{diam} A_{n}<\mathrm{d}(x, y)$ by left hypothesis 4
(d) $\Longrightarrow \exists n$ such that $\sup \left\{\mathrm{d}(x, y) \mid x, y \in A_{n}\right\}<\mathrm{d}(x, y)$
(e) This is a contradiction, so $\{x, y\} \notin A$ and $\left|\cap A_{n}\right|<2$.

(2) Proof that $\left|\cap A_{n}\right| \geq 1$ :
(a) Let $x_{n} \in A_{n}$ and $x_{m} \in A_{m}$
(b) $\forall \varepsilon, \exists N \in \mathbb{N}$ such that $A_{N}<\varepsilon$
(c) $\forall m, n>N, x_{n} \in A_{n} \subseteq A_{N}$ and $x_{m} \in A_{m} \subseteq A_{N}$
(d) $\mathrm{d}\left(x_{n}, x_{m}\right) \leq \operatorname{diam} A_{N}<\varepsilon \Longrightarrow\left\{x_{n}\right\}$ is a Cauchy sequence
(e) Because $\left\{x_{n}\right\}$ is complete, $x_{n} \rightarrow x$.
(f) $\Longrightarrow x \in\left(A_{n}\right)^{-}=A_{n}$
(g) $\Longrightarrow\left|A_{n}\right| \geq 1$

Definition 3.19 ${ }^{31}$ Let $(X, d)$ be a distance space. Let $C$ be the set of all convergent sequences in $(X, d)$. The distance function $\mathrm{d}$ is continuous in $(X, \mathrm{~d})$ if

$$
\left(x_{n}\right),\left(y_{n}\right) \in C \quad \Longrightarrow \quad \lim _{n \rightarrow \infty}\left(\mathrm{d}\left(x_{n}, y_{n}\right)\right)=\mathrm{d}\left(\lim _{n \rightarrow \infty}\left(x_{n}\right), \lim _{n \rightarrow \infty}\left(y_{n}\right)\right) \text {. }
$$

\section{A distance function is discontinuous if it is not continuous.}

\footnotetext{
${ }^{30}$ in metric space: $\boxminus$ [Davis(2005)], page $28, \otimes$ [Hausdorff(1937)], page 150

$31 \boxminus$ [Blumenthal(1953)] page 9 〈DEFINITION 6.3〉
} 
Remark 3.20 Rather than defining continuity of a distance function in terms of the sequential characterization of continuity as in Definition 3.19 (previous), we could define continuity using an inverse image characterization of continuity" (Definition 3.9 page 8). Assuming an equivalent topological space is used for both characterizations, the two characterizations are equivalent (Theorem A.20 page 29). In fact, one could construct an equivalence such as the following:

$$
\left\{\begin{array}{l}
\mathrm{d} \text { is continuous in } \mathbb{R}^{X^{2}} \\
\begin{array}{l}
\text { (Definition A.11 page 28) } \\
\text { (inverse image characterization of continuity) }
\end{array}
\end{array}\right\} \Longleftrightarrow\left\{\begin{array}{l}
\left(x_{n}\right),\left(y_{n}\right) \in C \quad \Longrightarrow \\
\lim _{n \rightarrow \infty}\left(\mathrm{d}\left(x_{n}, y_{n}\right)\right)=\mathrm{d}\left(\lim _{n \rightarrow \infty}\left(x_{n}\right), \lim _{n \rightarrow \infty}\left(y_{n}\right)\right) \\
\text { (Definition A.16 page 28) } \\
\text { (sequential characterization of continuity) }
\end{array}\right\}
$$

Note that just as $\left(x_{n}\right)$ is a sequence in $X$, so the ordered pair $\left(\left(x_{n}\right),\left(y_{n}\right)\right)$ is a sequence in $X^{2}$. The remainder follows from Theorem A.20 (page 29). However, use of the inverse image characterization is somewhat troublesome because we would need a topology on $X^{2}$, and we don't immediately have one defined and ready to use. In fact, we don't even immediately have a distance space on $X^{2}$ defined or even open balls in such a distance space. The result is, for the scope of this paper, it is arguably not worthwhile constructing the extra structure, but rather instead this paper uses the sequential characterization as a definition (as in Definition 3.19).

\subsection{Examples}

Similar distance functions and several of the observations for the examples in this section can be found in $\bullet$ [Blumenthal(1953)] pages 8-13.

In a metric space, all open balls are open, the open balls form a base for a topology, the limits of convergent sequences are unique, and the metric function is continuous. In the distance space of the next example, none of these properties hold.

Example 3.21 ${ }^{32}$ Let $(x, y)$ be an ordered pair in $\mathbb{R}^{2}$. Let $(a: b)$ be an open interval and $(a: b]$ a halfopen interval in $\mathbb{R}$. Let $|x|$ be the absolute value of $x \in \mathbb{R}$. The function $\mathrm{d}(x, y) \in \mathbb{R}^{\mathbb{R} \times \mathbb{R}}$ such that

$$
\mathrm{d}(x, y) \triangleq\left\{\begin{array}{lll}
y & \forall(x, y) \in\{4\} \times(0: 2] & \text { (vertical half-open interval) } \\
x & \forall(x, y) \in(0: 2] \times\{4\} & \text { (horizontal half-open interval) } \\
|x-y| & \text { otherwise } & \text { (Euclidean) }
\end{array}\right\} \quad \text { is a distance on } \mathbb{R} .
$$

Note some characteristics of the distance space $(\mathbb{R}, d)$ :

(1) $(\mathbb{R}, d)$ is not a metric space because d does not satisfy the triangle inequality:

$$
\mathrm{d}(0,4) \triangleq|0-4|=4 \not 2=|0-1|+1 \triangleq \mathrm{d}(0,1)+\mathrm{d}(1,4)
$$

(2) Not every open ball in $(\mathbb{R}, d)$ is open.

For example, the open ball $\mathrm{B}(3,2)$ is not open because $4 \in \mathrm{B}(3,2)$ but for all $0<\varepsilon<1$

$$
\mathrm{B}(4, \varepsilon)=(4-\varepsilon: 4+\varepsilon) \cup(0: \varepsilon) \nsubseteq(1: 5)=\mathrm{B}(3,2)
$$

(3) The open balls of $(\mathbb{R}, d)$ do not form a base for a topology on $\mathbb{R}$.

This follows directly from item (2) and Theorem 3.10 (page 8).

\footnotetext{
${ }^{32}$ A similar distance function $d$ and item (4) page 13 can in essence be found in $₫$ [Blumenthal(1953)] page 8. Definitions for Example 3.21: $(x, y)$ : Definition 2.3 (page 4); $(a: b)$ and $(a: b]$ : Definition 2.9 (page 5); $|x|$ : Definition 2.10 (page 5); $\mathbb{R}^{\mathbb{R} \times \mathbb{R}}$ : Definition 2.3 (page 4); distance: Definition 3.1 (page 6); open ball: Definition 3.5 (page 6); open: Definition 3.6 (page 7); base: Definition A.3 (page 27); topology: Definition A.1 (page 26); open set: Definition 3.6 (page 7); topological space induced by $(\mathbb{R}, \mathrm{d})$ : Definition 3.9 (page 8); discontinuous: Definition 3.19 (page 11);
} 
(4) In the distance space $(\mathbb{R}, \mathrm{d})$, limits are not unique;

For example, the sequence $(1 / n)_{1}^{\infty}$ converges both to the limit 0 and the limit 4 in $(\mathbb{R}, d)$ :

$$
\begin{aligned}
& \lim _{n \rightarrow \infty} \mathrm{d}\left(x_{n}, 0\right) \triangleq \lim _{n \rightarrow \infty} \mathrm{d}(1 / n, 0) \triangleq \lim _{n \rightarrow \infty}|1 / n-0|=0 \quad \Longrightarrow \quad(1 / n) \rightarrow 0 \\
& \lim _{n \rightarrow \infty} \mathrm{d}\left(x_{n}, 4\right) \triangleq \lim _{n \rightarrow \infty} \mathrm{d}(1 / n, 4) \triangleq \lim _{n \rightarrow \infty}(1 / n) \quad=0 \quad \Longrightarrow \quad(1 / n) \rightarrow 4
\end{aligned}
$$

(5) The topological space $(X, T)$ induced by $(\mathbb{R}, \mathrm{d})$ also yields limits of 0 and 4 for the sequence $(1 / n)_{1}^{\infty}$, just as it does in item (4). This is largely due to the fact that, for small $\varepsilon$, the open balls $\mathrm{B}(0, \varepsilon)$ and $\mathrm{B}(4, \varepsilon)$ are open.

$$
\begin{aligned}
\mathrm{B}(0, \varepsilon) \text { is open } & \Longrightarrow \text { for each } U \in T \text { that contains } 0, \exists N \in \mathbb{N} \quad \text { such that } \quad 1 / n \in U \quad \forall n>N \\
& \Longleftrightarrow(1 / n) \rightarrow 0 \quad \text { by definition of convergence (Definition A.16 page 28) } \\
\mathrm{B}(4, \varepsilon) \text { is open } & \Longrightarrow \text { for each } U \in T \text { that contains } 4, \exists N \in \mathbb{N} \quad \text { such that } \quad 1 / n \in U \quad \forall n>N \\
& \Longleftrightarrow(1 / n) \rightarrow 4 \quad \text { by definition of convergence (Definition A.16 page 28) }
\end{aligned}
$$

(6) The distance function $\mathrm{d}$ is discontinuous (Definition 3.19 page 11):

$$
\begin{aligned}
\lim _{n \rightarrow \infty}((\mathrm{d}(1-1 / n, 4-1 / n)) & =\lim _{n \rightarrow \infty}(|(1-1 / n)-(4-1 / n)|)=|1-4|=3 \neq 4=\mathrm{d}(0,4) \\
& =\mathrm{d}\left(\lim _{n \rightarrow \infty}(1-1 / n), \lim _{n \rightarrow \infty}(4-1 / n)\right)
\end{aligned}
$$

In a metric space, all convergent sequences are also Cauchy. However, this is not the case for all distance spaces, as demonstrated next:

Example 3.22 ${ }^{33}$ The function $\mathrm{d}(x, y) \in \mathbb{R}^{\mathbb{R} \times \mathbb{R}}$ such that

$$
\mathrm{d}(x, y) \triangleq\left\{\begin{array}{lll}
|x-y| & \text { for } x=0 \text { or } y=0 \text { or } x=y & \text { (Euclidean }) \\
1 & \text { otherwise } & \text { (discrete })
\end{array}\right\} \quad \text { is a distance on } \mathbb{R} .
$$

Note some characteristics of the distance space $(\mathbb{R}, d)$ :

(1) $(X, \mathrm{~d})$ is not a metric space because the triangle inequality does not hold:

$$
\mathrm{d}\left(\frac{1}{4}, \frac{1}{2}\right)=1 \not \leq \frac{3}{4}=\left|\frac{1}{4}-0\right|+\left|0-\frac{1}{2}\right|=\mathrm{d}\left(\frac{1}{4}, 0\right)+\mathrm{d}\left(0, \frac{1}{2}\right)
$$

(2) The open ball $\mathrm{B}\left(\frac{1}{4}, \frac{1}{2}\right)$ is not open because for any $\varepsilon \in \mathbb{R}^{+}$, no matter how small,

$$
\mathrm{B}(0, \varepsilon)=(-\varepsilon:+\varepsilon) \nsubseteq\left\{0, \frac{1}{4}\right\}=\left\{x \in X \mid \mathrm{d}\left(\frac{1}{4}, x\right)<\frac{1}{2}\right\} \triangleq \mathrm{B}\left(\frac{1}{4}, \frac{1}{2}\right)
$$

(3) Even though not all the open balls are open, it is still possible to have an open set in (X,d). For example, the set $U \triangleq\{1,2\}$ is open:

$$
\begin{aligned}
& \mathrm{B}(1,1) \triangleq\{x \in X \mid \mathrm{d}(1, x)<1\}=\{1\} \subseteq\{1,2\} \triangleq U \\
& \mathrm{~B}(2,1) \triangleq\{x \in X \mid \mathrm{d}(2, x)<1\}=\{2\} \subseteq\{1,2\} \triangleq U
\end{aligned}
$$

(4) By item (2) and Theorem 3.10 (page 8), the open balls of ( $\mathbb{R}, d$ ) do not form a base for a topology on $\mathbb{R}$.

(5) Even though the open balls in $(\mathbb{R}, \mathrm{d})$ do not induce a topology on $X$, it is still possible to find a set of open sets in $(X, \mathrm{~d})$ that $i$ a topology. For example, the set $\{\varnothing,\{1,2\}, \mathbb{R}\}$ is a topology on $\mathbb{R}$.

(6) In $(\mathbb{R}, d)$, limits of convergent sequences are unique:

$$
\left(x_{n}\right) \rightarrow x \Longrightarrow \lim _{n \rightarrow \infty} \mathrm{d}\left(x_{n}, x\right)=\left\{\begin{array}{rlll}
\lim \left|x_{n}-0\right| & =0 & \text { for } x=0 & \text { OR } \\
|x-x| & =0 & \text { for constant }\left(x_{n}\right) \text { for } n>N & \text { OR } \\
1 & \neq 0 & \text { otherwise }
\end{array}\right\}
$$

\footnotetext{
${ }^{33}$ The distance function $d$ and item (7) page 14 can in essence be found in $\boxminus$ [Blumenthal(1953)] page 9
} 
which says that there are only two ways for a sequence to converge: either $x=0$ or the sequence eventually becomes constant (or both). Any other sequence will diverge. Therefore we can say the following:

(a) If $x=0$ and the sequence is not constant, then the limit is unique and 0 .

(b) If $x=0$ and the sequence is constant, then the limit is unique and 0 .

(c) If $x \neq 0$ and the sequence is constant, then the limit is unique and $x$.

(d) If $x \neq 0$ and the sequence is not constant, then the sequence diverges and there is no limit.

(7) In $(\mathbb{R}, d)$, a convergent sequence is not necessarily Cauchy. For example,

(a) the sequence $(1 / n)_{n \in \mathbb{N}}$ is convergent with limit $0: \lim _{n \rightarrow \infty} \mathrm{d}(1 / n, 0)=\lim _{n \rightarrow \infty} 1 / n=0$

(b) However, even though $(1 / n)$ is convergent, it is not Cauchy: $\lim _{n, m \rightarrow \infty} \mathrm{d}(1 / n, 1 / m)=1 \neq 0$

(8) The distance function $\mathrm{d}$ is discontinuous in $(X, \mathrm{~d})$ :

$$
\lim _{n \rightarrow \infty}(\mathrm{d}(1 / n, 2-1 / n))=1 \neq 2=\mathrm{d}(0,2)=\mathrm{d}\left(\lim _{n \rightarrow \infty}(1 / n), \lim _{n \rightarrow \infty}(2-1 / n)\right) .
$$

Example 3.23 ${ }^{34}$ The function $d(x, y) \in \mathbb{R}^{\mathbb{R} \times \mathbb{R}}$ such that

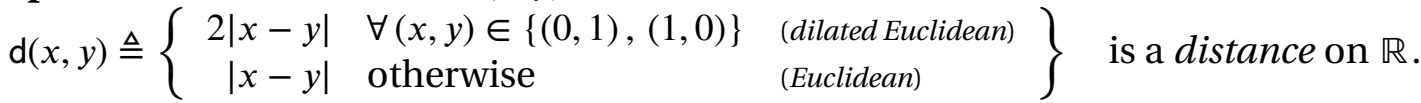

Note some characteristics of the distance space $(\mathbb{R}, \mathrm{d})$ :

(1) $(\mathbb{R}, \mathrm{d})$ is not a metric space because d does not satisfy the triangle inequality:

$$
\mathrm{d}(0,1) \triangleq 2|0-1|=2 \not \leq 1=|0-1 / 2|+|1 / 2-1| \triangleq \mathrm{d}(0,1 / 2)+\mathrm{d}(1 / 2,1)
$$

(2) The function $\mathrm{d}$ is discontinuous:

$$
\lim _{n \rightarrow \infty}(\mathrm{d}(1-1 / n, 1 / n)) \triangleq \lim _{n \rightarrow \infty}(|1-1 / n-1 / n|)=1 \neq 2=2|0-1| \triangleq \mathrm{d}(0,1)=\mathrm{d}\left(\lim _{n \rightarrow \infty}(1-1 / n), \lim _{n \rightarrow \infty}(1 / n)\right) .
$$

(3) In $(X, d)$, open balls are open:

(a) $\mathrm{p}(x, y) \triangleq|x-y|$ is a metric and thus all open balls in that do not contain both 0 and 1 are open.

(b) By Example C.4 (page 36), $\mathrm{q}(x, y) \triangleq 2|x-y|$ is also a metric and thus all open balls containing 0 and 1 only are open.

(c) The only question remaining is with regards to open balls that contain 0,1 and some other element(s) in $\mathbb{R}$. But even in this case, open balls are still open. For example:

$\mathrm{B}(-1,2)=(-1: 2)=(-1: 1) \cup(1: 2)$

Note that both $(-1: 1)$ and $(1: 2)$ are open, and thus by Theorem 3.7 (page 7), $\mathrm{B}(-1,2)$ is open as well.

(4) By item (3) and Theorem 3.10 (page 8), the open balls of ( $\mathbb{R}, d$ ) do form a base for a topology on $\mathbb{R}$.

(5) In $(X, d)$, the limits of convergent sequences are unique. This is demonstrated in Example 4.22 (page 25) using additional structure developed in Section 4.

(6) In $(X, \mathrm{~d})$, convergent sequences are Cauchy. This is also demonstrated in Example 4.22 (page 25).

\footnotetext{
${ }^{34}$ The distance function $d$ and item (2) page 14 can in essence be found in $₫$ [Blumenthal(1953)] page 9
} 
The distance functions in Example 3.21 (page 12)-Example 3.23 (page 14) were all discontinuous. In the absence of the triangle inequality and in light of these examples, one might try replacing the triangle inequality with the weaker requirement of continuity. However, as demonstrated by the next example, this also leads to an arguably disastrous result.

Example 3.24 ${ }^{35}$ The function $\mathrm{d} \in \mathbb{R}^{\mathbb{R} \times \mathbb{R}}$ such that $\mathrm{d}(x, y) \triangleq(x-y)^{2}$ is a distance on $\mathbb{R}$. Note some characteristics of the distance space $(\mathbb{R}, d)$ :

(1) $(\mathbb{R}, \mathrm{d})$ is not a metric space because the triangle inequality does not hold: $\mathrm{d}(0,2) \triangleq(0-2)^{2}=4 \not \leq 2=(0-1)^{2}+(1-2)^{2} \triangleq \mathrm{d}(0,1)+\mathrm{d}(1,2)$

(2) The distance function $\mathrm{d}$ is continuous in $(X, \mathrm{~d})$. This is demonstrated in the more general setting of Section 4 in Example 4.23 (page 25).

(3) Calculating the length of curves in $(X, \mathrm{~d})$ leads to a paradox: ${ }^{36}$

(a) Partition $[0: 1]$ into $2^{N}$ consecutive line segments connected at the points

$$
\left(0, \frac{1}{2^{N}}, \frac{2}{2^{N}}, \frac{3}{2^{N}}, \ldots, \frac{2^{N-1} 1}{2^{N}}, 1\right)
$$

(b) Then the distance, as measured by d, between any two consecutive points is

$$
\mathrm{d}\left(p_{n}, p_{n+1}\right) \triangleq\left(p_{n}-p_{n+1}\right)^{2}=\left(\frac{1}{2^{N}}\right)^{2}=\frac{1}{2^{2 N}}
$$

(c) But this leads to the paradox that the total length of $[0: 1]$ is 0 :

$$
\lim _{N \rightarrow \infty} \sum_{n=0}^{2^{N}-1} \frac{1}{2^{2 N}}=\lim _{N \rightarrow \infty} \frac{2^{N}}{2^{2 N}}=\lim _{N \rightarrow \infty} \frac{1}{2^{N}}=0
$$

\section{Distance spaces with power triangle inequalities}

\subsection{Definitions}

This paper introduces a new relation called the power triangle inequality (Definition 4.3 page 16). It is a generalization of other common relations, including the triangle inequality (Definition 4.4 page 16). The power triangle inequality is defined in terms of a function herein called the power triangle function (next definition). This function is a special case of the power mean with $N=2$ and $\lambda_{1}=\lambda_{2}=\frac{1}{2}$ (Definition B.6 page 31). Power means have the attractive properties of being continuous and strictly monontone with respect to a free parameter $p \in \mathbb{R}^{*}$ (Theorem B.7 page 31). This fact is inherited and exploited by the power triangle inequality (Corollary 4.6 page 16).

Definition 4.1 Let $(X, \mathrm{~d})$ be a distance space (Definition 3.1 page 6 ). Let $\mathbb{R}^{+}$be the set of all positive real numbers and $\mathbb{R}^{*}$ be the set of extended real numbers (Definition 2.1 page 4). The power triangle function $\tau$ on $(X, \mathrm{~d})$ is defined as

$$
\tau(p, \sigma ; x, y, z ; \mathrm{d}) \triangleq 2 \sigma\left[\frac{1}{2} \mathrm{~d}^{p}(x, z)+\frac{1}{2} \mathrm{~d}^{p}(z, y)\right]^{\frac{1}{p}} \quad \forall(p, \sigma) \in \mathbb{R}^{*} \times \mathbb{R}^{\vdash}, \quad x, y, z \in X
$$

\footnotetext{
$35 \boxminus$ [Blumenthal(1953)] pages 12-13, $\boxminus[$ Laos(1998)] pages 118-119

${ }^{36}$ This is the method of "inscribed polygons" for calculating the length of a curve and goes back to Archimedes: $\otimes$ [Brunschwig et al.(2003)Brunschwig, Lloyd, and Pellegrin] page 26, $\theta$ [Walmsley(1920)], page $200\langle \$ 158\rangle$,
} 
Remark 4.2 ${ }^{37}$ In the field of probabilistic metric spaces, a function called he triangle function was introduced by Sherstnev in 1962. However, the power triangle function as defined in this present paper is not a special case of (is not compatible with) the triangle function of Sherstnev. Another definition of triangle function has been offered by Bessenyei in 2014 with special cases of $\Phi(u, v) \triangleq c(u+v)$ and $\Phi(u, v) \triangleq\left(u^{p}+v^{p}\right)^{\frac{1}{p}}$, which are similar to the definition of power triangle function offered in this present paper.

Definition 4.3 Let $(X, \mathrm{~d})$ be a distance space. Let $2^{X X X}$ be the set of all trinomial relations (Definition 2.3 page 4) on $X$. A relation $\otimes(p, \sigma ; \mathrm{d})$ in $2^{X X X}$ is a power triangle inequality on $(X, \mathrm{~d})$ if

$\otimes(p, \sigma ; \mathrm{d}) \triangleq\left\{(x, y, z) \in X^{3} \mid \mathrm{d}(x, y) \leq \tau(p, \sigma ; x, y, z ; \mathrm{d})\right\} \quad$ for some $(p, \sigma) \in \mathbb{R}^{*} \times \mathbb{R}^{+}$.

The tupple $(X, \mathrm{~d}, p, \sigma)$ is a power distance space and $\mathrm{d}$ a power distance or power distance function if $(X, \mathrm{~d})$ is a distance space in which the triangle relation $\otimes(p, \sigma ; \mathrm{d})$ holds.

The power triangle function can be used to define some standard inequalities (next definition). See Corollary 4.7 (page 17) for some justification of the definitions.

Definition 4.4 ${ }^{38}$ Let $\otimes(p, \sigma ; d)$ be a power triangle inequality on a distance space $(X, d)$.

1. $\otimes(\infty, \sigma / 2 ; d)$ is the $\boldsymbol{\sigma}$-inframetric inequality $\quad 6$. $\otimes(1 / 2,2 ; d)$ is the square mean root inequality

2. $\otimes\left(\infty, \frac{1}{2} ; d\right)$ is the inframetric inequality

3. $\otimes(2, \sqrt{2} h ; d)$ is the quadratic inequality

4. $\otimes(1, \sigma ; d)$ is the relaxed triangle inequality $9 . \otimes\left(-\infty, \frac{1}{2} ; d\right)$ is the minimal inequality

5. $\otimes(1,1 ; \mathrm{d})$ is the triangle inequality

Definition 4.5 39 Let $(X, \mathrm{~d})$ be a distance space (Definition 3.1 page 6$)$.

$\begin{array}{lllll}\text { 1. } & (X, \mathrm{~d}) \text { is a } & \text { metric space } & \text { if the triangle inequality } & \text { holds in } X . \\ \text { 2. } & (X, \mathrm{~d}) \text { is a } & \text { near metric space } & \text { if the relaxed triangle inequality } & \text { holds in } X . \\ \text { 3. } & (X, \mathrm{~d}) \text { is an } & \text { inframetric space } & \text { if the inframetric inequality } & \text { holds in } X . \\ \text { 4. } & (X, \mathrm{~d}) \text { is a } & \boldsymbol{\sigma} \text {-inframetric space } & \text { if the } \sigma \text {-inframetric inequality } & \text { holds in } X .\end{array}$

\subsection{Properties}

\subsubsection{Relationships of the power triangle function}

Corollary 4.6 Let $\tau(p, \sigma ; x, y, z$; d) be the POWER TRIANGLE FUNCTION (Definition 4.1 page 15) in the DISTANCE SPACE (Definition 3.1 page 6$)(X, \mathrm{~d})$. Let $(\mathbb{R},|\cdot|, \leq)$ be the ORDERED METRIC SPACE with the usual ordering relation

\footnotetext{
$37 \otimes[$ Sherstnev(1962)], page 4, $\otimes$ [Schweizer and Sklar(1983)] page $9\langle(1.6 .1)-(1.6 .4)\rangle$, 圈 [Bessenyei and Pales(2014)] page 2

${ }^{38}$ 圈 [Bessenyei and Pales(2014)] page 2, 圊 [Czerwik(1993)] page $5\langle$ b-metric; (1),(2),(5)〉, 圈 [Fagin et al.(2003a)Fagin, Kumar, and Sivakumar], 圈 [Fagin et al.(2003b)Fagin, Kumar, and Sivakumar] 〈Definition 4.2 (Relaxed metrics) $\rangle$, 圈 [Xia(2009)] page 453〈Definition 2.1〉, $\boxminus$ [Heinonen(2001)] page $109\langle 14.1$ Quasi-

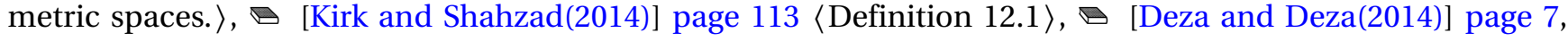
圈 [Hoehn and Niven(1985)] page 151, $₫$ [Gibbons et al.(1977)Gibbons, Olkin, and Sobel] page $51<$ square-mean$\operatorname{root}(S M R)(2.4 .1)\rangle, \otimes[$ Euclid(circa 300BC)] 〈triangle inequality-Book I Proposition 20

${ }^{39}$ metric space: $\otimes$ [Dieudonné(1969)], page 28, $\bullet[$ Copson(1968)], page 21, $\bullet$ [Hausdorff(1937)] page 109, $\otimes$ [Fréchet(1928)], 圈 [Fréchet(1906)] page 30 near metric space: 圈 [Czerwik(1993)] page $5\langle b$-metric; (1),(2),(5) $\rangle$, 圈 [Fagin et al.(2003a)Fagin, Kumar, and Sivakumar], 圈 [Fagin et al.(2003b)Fagin, Kumar, and Sivakumar] 〈Definition 4.2 (Relaxed metrics) $\rangle$, 圈 [Xia(2009)] page 453〈Definition 2.1〉, $\bullet$ [Heinonen(2001)] page 109 $\langle 14.1$ Quasimetric spaces. $\rangle, \otimes[$ Kirk and Shahzad(2014)] page $113\langle$ Definition 12.1〉, $\bullet$ [Deza and Deza(2014)] page 7
} 
$\leq$ and usual metric $|\cdot|$ on $\mathbb{R}$. The function $\tau(p, \sigma ; x, y, z ; d)$ is CONTINUOUs and strictLy Monotone in $(\mathbb{R},|\cdot|, \leq)$ with respect to both the variables $p$ and $\sigma$.

QPROOF:

(1) Proof that $\tau(p, \sigma ; x, y, z ; \mathrm{d})$ is continuous and strictly monotone with respect to $p$ : This follows directly from Theorem B.7 (page 31).

(2) Proof that $\tau(p, \sigma ; x, y, z ; \mathrm{d})$ is continuous and strictly monotone with respect to $\sigma$ :

$$
\begin{array}{rlrl}
\tau(p, \sigma ; x, y, z ; \mathrm{d}) & \triangleq 2 \sigma \underbrace{\left[\frac{1}{2} \mathrm{~d}^{p}(x, z)+\frac{1}{2} \mathrm{~d}^{p}(z, y)\right]^{\frac{1}{p}}}_{\mathrm{f}(p, x, y, z)} \quad \text { by definition of } \tau \text { (Definition 4.1 page 15) } \\
& =2 \sigma \mathrm{f}(p, x, y, z) & \text { where } \mathrm{f} \text { is defined as above } \\
& \Longrightarrow \tau \text { is affine with respect to } \sigma \quad \\
& \Longrightarrow \tau \text { is continuous } \text { and strictly monotone with respect to } \sigma:
\end{array}
$$

Corollary 4.7 Let $\tau(p, \sigma ; x, y, z ; \mathrm{d})$ be the POWER TRIANGLE FUNCTION in the DISTANCE SPACE (Definition 3.1 page 6) $(X, \mathrm{~d})$.

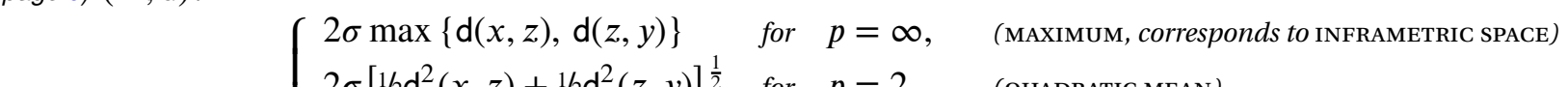

$$
\begin{aligned}
& \tau(p, \sigma ; x, y, z ; \mathrm{d})=\left\{\begin{array}{lll}
2 \sigma\left[1 / 2 \mathrm{~d}^{2}(x, z)+1 / 2 \mathrm{~d}^{2}(z, y)\right]^{\frac{1}{2}} & \text { for } p=2, & \text { (QuAdratic MEAN) } \\
\sigma[\mathrm{d}(x, z)+\mathrm{d}(z, y)] & \text { for } p=1, & \text { (ARITHMETIC MEAN, corresponds to NeAR METRIC SPACE) } \\
2 \sigma \sqrt{\mathrm{d}(x, z)} \sqrt{\mathrm{d}(z, y)} & \text { for } p=0 & \text { (GEOMETRIC MEAN) } \\
4 \sigma\left[\frac{1}{\mathrm{~d}(x, z)}+\frac{1}{\mathrm{~d}(z, y)}\right]^{-1} & \text { for } p=-1 & \text { (HARMONiC MEAN) } \\
2 \sigma \min \{\mathrm{d}(x, z), \mathrm{d}(z, y)\} & \text { for } p=-\infty, & \text { (Minimum) }
\end{array}\right.
\end{aligned}
$$

QRoof: These follow directly from Theorem B.7 (page 31).

Corollary 4.8 Let $(X, \mathrm{~d})$ be $a$ DISTANCE SPACE.

$$
\begin{aligned}
2 \sigma \min \{\mathrm{d}(x, z), \mathrm{d}(z, y)\} & \leq 4 \sigma\left[\frac{1}{\mathrm{~d}(x, z)}+\frac{1}{\mathrm{~d}(z, y)}\right]^{-1} \leq 2 \sigma \sqrt{\mathrm{d}(x, z)} \sqrt{\mathrm{d}(z, y)} \\
& \leq \sigma[\mathrm{d}(x, z)+\mathrm{d}(z, y)] \leq 2 \sigma \max \{\mathrm{d}(x, z), \mathrm{d}(z, y)\}
\end{aligned}
$$

QRoof: These follow directly from Corollary B.8 (page 34).

\subsubsection{Properties of power distance spaces}

The power triangle inequality property of a power distance space axiomatically endows a metric with an upper bound. Lemma 4.9 (next) demonstrates that there is a complementary lower bound somewhat similar in form to the power triangle inequality upper bound. In the special case where $2 \sigma=2^{\frac{1}{p}}$, the lower bound helps provide a simple proof of the continuity of a large class of power distance functions (Theorem 4.18 page 23). The inequality $2 \sigma \leq 2^{\frac{1}{p}}$ is a special relation in this paper and appears repeatedly in this paper; it appears as an inequality in Lemma 4.13 (page 20), Corollary 4.12 (page 20) and Corollary 4.14 (page 21), and as an equality in Lemma 4.9 (next) and Theorem 4.18 (page 23). It is plotted in Figure 1 (page 18). 


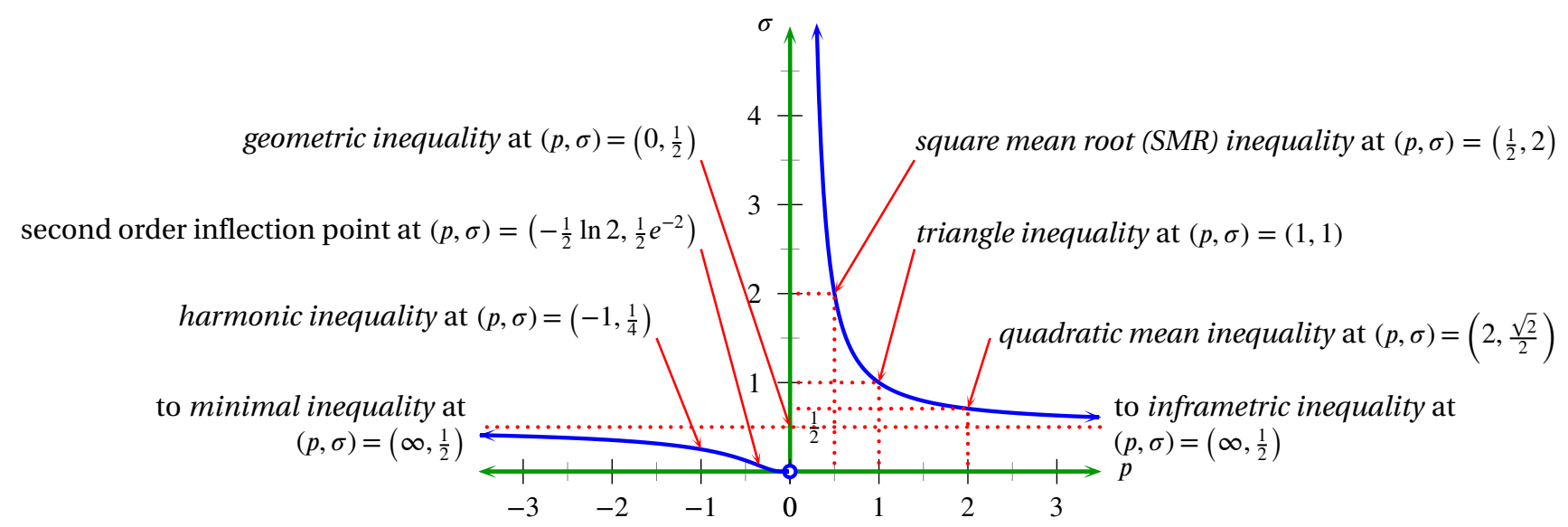

Figure 1: $\sigma=\frac{1}{2}\left(2^{\frac{1}{p}}\right)=2^{\frac{1}{p}-1}$ or $p=\frac{\ln 2}{\ln (2 \sigma)}$ (see Lemma 4.9 page 18, Lemma 4.13 page 20, Corollary 4.14 page 21, Corollary 4.12 page 20, and Theorem 4.18 page 23 ).

Lemma 4.9 ${ }^{40}$ Let $(X, \mathrm{~d}, p, \sigma)$ be a POWER TRIANGLE TRIANGLE SPACE (Definition 4.3 page 16$)$. Let $|\cdot|$ be the ABSOLUTE VALUE function (Definition 2.10 page 5). Let $\max \{x, y\}$ be the maximum and $\min \{x, y\}$ the minimum of any $x, y \in \mathbb{R}^{*}$. Then, for all $(p, \sigma) \in \mathbb{R}^{*} \times \mathbb{R}^{+}$,

$$
\begin{aligned}
& \text { 1. } \mathrm{d}^{p}(x, y) \geq \max \left\{0, \frac{2}{(2 \sigma)^{p}} \mathrm{~d}^{p}(x, z)-\mathrm{d}^{p}(z, y), \frac{2}{(2 \sigma)^{p}} \mathrm{~d}^{p}(y, z)-\mathrm{d}^{p}(z, x)\right\} \quad \forall x, y, z \in X \quad \text { and } \\
& \text { 2. } \mathrm{d}(x, y) \geq|\mathrm{d}(x, z)-\mathrm{d}(z, y)| \quad \text { if } p \neq 0 \text { and } 2 \sigma=2^{\frac{1}{p}} \quad \forall x, y, z \in X \text {. }
\end{aligned}
$$

QPROOF:

(1) lemma: $\frac{2}{(2 \sigma)^{p}} \mathrm{~d}^{p}(x, z)-\mathrm{d}^{p}(z, y) \leq \mathrm{d}^{p}(x, y) \quad \forall(p, \sigma) \in \mathbb{R}^{*} \times \mathbb{R}^{+}$: Proof:

$$
\begin{array}{rlrl}
\frac{2}{(2 \sigma)^{p}} \mathrm{~d}^{p}(x, z)-\mathrm{d}^{p}(z, y) & \leq \frac{2}{(2 \sigma)^{p}}\left[2 \sigma\left[1 / 2 \mathrm{~d}^{p}(x, y)+{ }^{1} / 2 \mathrm{~d}^{p}(y, z)\right]^{\frac{1}{p}}\right]^{p}-\mathrm{d}^{p}(z, y) & & \text { by power triangle inequality } \\
& =\frac{2(2 \sigma)^{p}}{(2 \sigma)^{p}}\left[1 / 2 \mathrm{~d}^{p}(x, y)+{ }^{1} / 2 \mathrm{~d}^{p}(y, z)\right]-\mathrm{d}^{p}(z, y) & \\
& =\left[\mathrm{d}^{p}(x, y)+\mathrm{d}^{p}(y, z)\right]-\mathrm{d}^{p}(y, z) & & \text { by symmetric property of } \mathrm{d} \\
& =\mathrm{d}^{p}(x, y) & &
\end{array}
$$

(2) Proof for $(p, \sigma) \in \mathbb{R}^{*} \times \mathbb{R}^{+}$case:

$$
\begin{array}{lll}
\mathrm{d}^{p}(x, y) & \geq \frac{2}{(2 \sigma)^{p}} \mathrm{~d}^{p}(x, z)-\mathrm{d}^{p}(z, y) & \text { by (1) lemma } \\
\mathrm{d}^{p}(x, y)=\mathrm{d}^{p}(y, x) & \geq \frac{2}{(2 \sigma)^{p}} \mathrm{~d}^{p}(y, z)-\mathrm{d}^{p}(z, x) & \text { by commutative property of } \mathrm{d} \text { and (1) lemma } \\
\mathrm{d}^{p}(x, y) & \geq 0 & \text { by non-negative property of } \mathrm{d} \text { (Definition } 3.1 \text { page 6) }
\end{array}
$$

The rest follows because $\mathrm{g}(x) \triangleq x^{\frac{1}{p}}$ is strictly monotone in $\mathbb{R}^{\mathbb{R}}$.

\footnotetext{
${ }^{40}$ in metric space $((p, \sigma)=(1,1)): \otimes$ [Dieudonné(1969)] page 28, $\boxminus$ [Michel and Herget(1993)] page 266, $\ominus[$ Berberian(1961)] page $37\langle$ Theorem II.4.1〉
} 
(3) Proof for $2 \sigma=2^{\frac{1}{p}}$ case:

$$
\begin{aligned}
\mathrm{d}(x, y) & \geq \max \left\{0, \frac{2}{(2 \sigma)^{p}} \mathrm{~d}^{p}(x, z)-\mathrm{d}^{p}(z, y), \frac{2}{(2 \sigma)^{p}} \mathrm{~d}^{p}(y, z)-\mathrm{d}^{p}(z, x)\right\}^{\frac{1}{p}} \\
& =\max \{0, \mathrm{~d}(x, z)-\mathrm{d}(z, y), \mathrm{d}(y, z)-\mathrm{d}(z, x)\} \\
& =\max \{0,(\mathrm{~d}(x, z)-\mathrm{d}(z, y)),-(\mathrm{d}(x, z)-\mathrm{d}(z, y))\} \\
& =|(\mathrm{d}(x, z)-\mathrm{d}(z, y))|
\end{aligned}
$$

by item (2) (page 18) by $2 \sigma=2^{\frac{1}{p}}$ hypothesis $\Longleftrightarrow \frac{2}{(2 \sigma)^{p}}=1$ by symmetric property of $d$

Theorem 4.10 Let $(X, \mathrm{~d}, p, \sigma)$ be a POWER DISTANCE SPACE (Definition 4.3 page 16). Let $\mathrm{B}$ be an OPEN BALL (Definition 3.5 page 6) on $(X, \mathrm{~d})$. Then for all $(p, \sigma) \in\left(\mathbb{R}^{*} \backslash\{0\}\right) \times \mathbb{R}^{+}$,

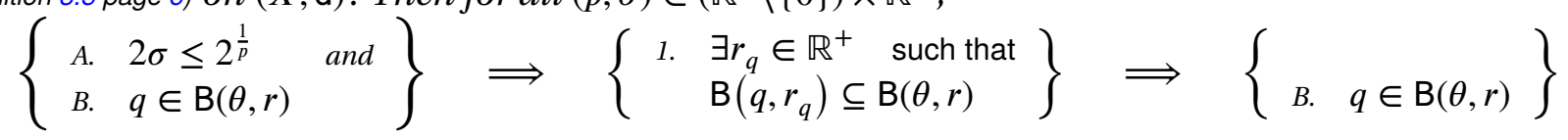

QPRoOF:

(1) lemma:

$$
\begin{aligned}
& q \in \mathrm{B}(\theta, r) \Longleftrightarrow \mathrm{d}(\theta, q)<r \\
& \Longleftrightarrow 0<r-\mathrm{d}(\theta, q) \\
& \Longleftrightarrow \exists r_{q} \in \mathbb{R}^{+} \text {such that } 0<r_{q}<r-\mathrm{d}(\theta, q)
\end{aligned}
$$

(2) Proof that $(A),(B) \Longrightarrow(1)$ :

$$
\begin{aligned}
\mathrm{B}\left(q, r_{q}\right) & \triangleq\left\{x \in X \mid \mathrm{d}(q, x)<r_{q}\right\} & & \text { by definition of open ball (Definition 3.5 page 6) } \\
& =\left\{x \in X \mid \mathrm{d}^{p}(q, x)<r_{q}^{p} \in \mathbb{R}^{+}\right\} & & \text {because } \mathrm{f}(x) \triangleq x^{p} \text { is monotone } \\
& \subseteq\left\{x \in X \mid \mathrm{d}^{p}(q, x)<r^{p}-\mathrm{d}^{p}(\theta, q)\right\} & & \text { by hypothesis B and (1) lemma page 19 } \\
& =\left\{x \in X \mid \mathrm{d}^{p}(\theta, q)+\mathrm{d}^{p}(q, x)<r^{p}\right\} & & \text { by field property of real numbers } \\
& =\left\{x \in X \mid\left[\mathrm{d}^{p}(\theta, q)+\mathrm{d}^{p}(q, x)\right]^{\frac{1}{p}}<r\right\} & & \text { because } \mathrm{f}(x) \triangleq x^{\frac{1}{p}} \text { is monotone } \\
& \subseteq\left\{x \in X \mid 2^{1-1 / p} \sigma\left[\mathrm{d}^{p}(\theta, q)+\mathrm{d}^{p}(q, x)\right]^{\frac{1}{p}}<r\right\} & & \text { by hypothesis A which implies } 2^{1-1 / p} \sigma \leq 1 \\
& =\left\{x \in X \mid 2 \sigma\left[1 / 2 \mathrm{~d}(\theta, q) x+{ }^{1 / 2} \mathrm{~d}^{p}(q, x)\right]^{\frac{1}{p}}<r\right\} & & \text { because } 2^{1-1 / p} \sigma=2 \sigma\left(\frac{1}{2}\right)^{\frac{1}{p}} \\
& \triangleq\{x \in X \mid \tau(p, \sigma, \theta, x, q)<r\} & & \text { by definition of } \tau \text { (Definition 4.1 page 15) } \\
& \subseteq\{x \in X \mid \mathrm{d}(\theta, x)<r\} & & \text { by definition of }(X, \mathrm{~d}, p, \sigma) \text { (Definition 4.3 page 16) } \\
& \triangleq \mathrm{B}(\theta, r) & & \text { by definition of open ball (Definition 3.5 page 6) }
\end{aligned}
$$

(3) Proof that $(B) \Longleftarrow(1)$ :

$$
\begin{aligned}
q & \in\{x \in X \mid \mathrm{d}(q, x)=0\} & & \text { by nondegenerate property (Definition 3.1 page 6) } \\
& \subseteq\left\{x \in X \mid \mathrm{d}(q, x)<r_{q}\right\} & & \text { because } r_{q}>0 \\
& \triangleq \mathrm{B}\left(q, r_{q}\right) & & \text { by definition of } \text { open ball (Definition 3.5 page 6) } \\
& \subseteq \mathrm{B}(\theta, r) & & \text { by hypothesis } 2
\end{aligned}
$$

${ }^{41} \boxminus$ [Aliprantis and Burkinshaw(1998)] page 17 (Theorem 3.3 (“The Archimedean Property") and Theorem $3.4\rangle, \boxminus[Z o r i c h(2004)]$ page $53\left\langle 6^{\circ}\right.$ (“The principle of Archimedes”) and $\left.7^{\circ}\right\rangle$ 
Corollary 4.11 Let $(X, \mathrm{~d}, p, \sigma)$ be a POWER DISTANCE SPACE. Then for all $(p, \sigma) \in\left(\mathbb{R}^{*} \backslash\{0\}\right) \times \mathbb{R}^{+}$, $\left\{2 \sigma \leq 2^{\frac{1}{p}}\right\} \quad \Longrightarrow \quad$ every OPEN BALL in $(X, \mathrm{~d})$ is OPEN $\}$

QRoof: This follows from Theorem 4.10 (page 19) and Theorem 3.10 (page 8).

Corollary 4.12 Let $(X, \mathrm{~d}, p, \sigma)$ be a POWER DistANCE SPACE. Let $\boldsymbol{B}$ be the set of all OPEN BALls in $(X, \mathrm{~d})$. Then for all $(p, \sigma) \in\left(\mathbb{R}^{*} \backslash\{0\}\right) \times \mathbb{R}^{+}$,

$$
\left\{2 \sigma \leq 2^{\frac{1}{p}}\right\} \quad \Longrightarrow \quad\{\text { B is a BASE for }(X, T)\}
$$

PROOF:

(1) The set of all open balls in $(X, \mathrm{~d})$ is a base for $(X, T)$ by Corollary 4.11 (page 20) and Theorem A.4 (page 27).

(2) $T$ is a topology on $X$ by Definition A.3 (page 27).

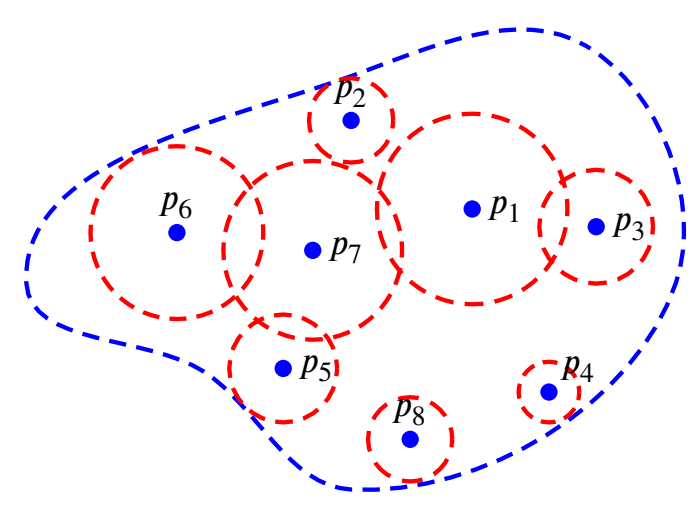

Figure 2: open set (see Lemma 4.13 page 20)

Lemma 4.13 (next) demonstrates that every point in an open set is contained in an open ball that is contained in the original open set (see also Figure 2 page 20).

Lemma 4.13 Let $(X, \mathrm{~d}, p, \sigma)$ be a POWER DISTANCE SPACE. Let B be an OPEN BALL on $(X, \mathrm{~d})$. Then for all $(p, \sigma) \in\left(\mathbb{R}^{*} \backslash\{0\}\right) \times \mathbb{R}^{+}$,

$\left\{\begin{array}{ll}\text { A. } & 2 \sigma \leq 2^{\frac{1}{p}} \text { and } \\ \text { B. } & U \text { is } \operatorname{OPEN} \operatorname{in}(X, \mathrm{~d})\end{array}\right\} \Longrightarrow\left\{\begin{array}{ll}1 . & \forall x \in U, \exists r \in \mathbb{R}^{+} \text {such that } \\ \mathrm{B}(x, r) \subseteq U\end{array}\right\} \Longrightarrow\left\{\begin{array}{ll}B . & U \text { is } \\ \operatorname{OPEN} \operatorname{in}(X, \mathrm{~d})\end{array}\right\}$

Proof:

(1) Proof that for $((A),(B) \Longrightarrow(1))$ :

$$
\begin{aligned}
U & =\bigcup\left\{\mathrm{B}\left(x_{\gamma}, r_{\gamma}\right) \mid \mathrm{B}\left(x_{\gamma}, r_{\gamma}\right) \subseteq U\right\} & & \text { by left hypothesis and Corollary } 4.12 \text { page } 20 \\
& \supseteq \mathrm{B}(x, r) & & \text { because } x \text { must be in one of those balls in } U
\end{aligned}
$$


(2) Proof that $((B) \Longleftarrow(1))$ case:

$$
\begin{aligned}
U & =\bigcup\{x \in X \mid x \in U\} & & \text { by definition of union operation } \bigcup \\
& =\bigcup\{\mathrm{B}(x, r) \mid x \in U \text { and } \mathrm{B}(x, r) \subseteq U\} & & \text { by hypothesis (1) } \\
& \Longrightarrow U \text { is open } & & \text { by Corollary 4.12 page 20 and Corollary } 3.8 \text { page } 8
\end{aligned}
$$

Corollary 4.14 ${ }^{42}$ Let $(X, \mathrm{~d}, p, \sigma)$ be a POWER DISTANCE SPACE. Let B be an OPEN BALL on $(X, \mathrm{~d})$. Then for all $(p, \sigma) \in\left(\mathbb{R}^{*} \backslash\{0\}\right) \times \mathbb{R}^{+}$,

$$
\left\{2 \sigma \leq 2^{\frac{1}{p}}\right\} \quad \Longrightarrow \quad\{\text { every open BaLl } \mathrm{B}(x, r) \text { in }(X, \mathrm{~d}) \text { is OPEN }\}
$$

QRoOF:

The union of any set of open balls is open

by Corollary 4.12 page 20

$\Longrightarrow$ the union of a set of just one open ball is open

$\Longrightarrow$ every open ball is open.

Theorem 4.15 ${ }^{43}$ Let $(X, \mathrm{~d}, p, \sigma)$ be a POWER DISTANCE SPACE. Let $(X, T)$ be $a$ TOPOLOGICAL SPACE IN$\operatorname{DUCED}$ BY $(X, \mathrm{~d})$. Let $\left(x_{n} \in X\right)_{n \in \mathbb{Z}}$ be a sequence in $(X, \mathrm{~d})$.

$$
\underbrace{\left(x_{n}\right) \text { converges to a limit } x}_{\text {(Definition A.16 page } 28 \text { ) }} \Longleftrightarrow\left\{\begin{array}{l}
\text { for any } \varepsilon \in \mathbb{R}^{+}, \text {there exists } N \in \mathbb{Z} \\
\text { such that for all } n>N, \quad \mathrm{~d}\left(x_{n}, x\right)<\varepsilon
\end{array}\right\}
$$

QPRoof:

$$
\begin{aligned}
\left(x_{n}\right) \rightarrow x & \Longleftrightarrow x_{n} \in U \quad \forall U \in N_{x}, n>N & & \text { by Definition A.16 page } 28 \\
& \Longleftrightarrow \exists \mathrm{B}(x, \varepsilon) \quad \text { such that } \quad x_{n} \in \mathrm{B}(x, \varepsilon) \forall n>N & & \text { by Lemma } 4.13 \text { page } 20 \\
& \Longleftrightarrow \mathrm{d}\left(x_{n}, x\right)<\varepsilon & & \text { by Definition } 3.5 \text { page } 6
\end{aligned}
$$

In distance spaces (Definition 3.1 page 6), not all convergent sequences are Cauchy (Example 3.22 page 13). However in a distance space with any power triangle inequality (Definition 4.3 page 16), all convergent sequences are Cauchy (next theorem).

Theorem 4.16 ${ }^{44} \operatorname{Let}(X, \mathrm{~d}, p, \sigma)$ be a POWER DisTANCE SPACE. Let $\mathrm{B}$ be an OPEn Ball on $(X, \mathrm{~d})$. For any $(p, \sigma) \in \mathbb{R}^{*} \times \mathbb{R}^{+}$,

$$
\left\{\begin{array}{l}
\left(x_{n}\right) \text { is CONVERGENT } \\
\text { in }(X, \mathrm{~d})
\end{array}\right\} \Longrightarrow\left\{\begin{array}{l}
\left(x_{n}\right) \text { is CAUCHY } \\
\text { in }(X, \mathrm{~d})
\end{array}\right\} \Longrightarrow\left\{\begin{array}{l}
\left(x_{n}\right) \text { is BOUNDED } \\
i n(X, \mathrm{~d})
\end{array}\right\}
$$

\footnotetext{
${ }^{42}$ in metric space $((p, \sigma)=(1,1)): \boxminus$ [Rosenlicht(1968)] pages 40-41, $\boxminus$ [Aliprantis and Burkinshaw(1998)] page 35

${ }^{43}$ in metric space: $\bullet[$ [Rosenlicht(1968)] page 45, $\bullet[$ Giles(1987)] page 37 〈3.2 Definition〉

${ }^{44}$ in metric space: $\boxminus$ [Giles(1987)] page $49\langle$ Theorem 3.30〉, $\boxminus$ [Rosenlicht(1968)] page 51, $\boxminus$ [Apostol(1975)] pages $72-73\langle$ Theorem 4.6$\rangle$
} 
QPRoOF:

(1) Proof that convergent $\Longrightarrow$ Cauchy:

$$
\begin{aligned}
\mathrm{d}\left(x_{n}, x_{m}\right) & \leq \tau\left(p, \sigma ; x_{n}, x_{m}, x\right) & & \text { by definition of power triangle inequality (Definition 4.3 page 16) } \\
& \triangleq 2 \sigma\left[\frac{1}{2} \mathrm{~d}^{p}\left(x_{n}, x\right)+\frac{1}{2} \mathrm{~d}^{p}\left(x_{m}, x\right)\right]^{\frac{1}{p}} & & \text { by definition of power triangle function (Definition 4.1 page 15) } \\
& <2 \sigma\left[\frac{1}{2} \varepsilon^{p}+\frac{1}{2} \varepsilon^{p}\right]^{\frac{1}{p}} & & \text { by convergence hypothesis (Definition A.16 page 28) } \\
& =2 \sigma \varepsilon & & \text { by definition of convergence (Definition A.16 page 28) } \\
& \Longrightarrow \text { Cauchy } & & \text { by definition of Cauchy (Definition 3.12 page 9) } \\
\mathrm{d}\left(x_{n}, x_{m}\right) & \leq \tau\left(\infty, \sigma ; x_{n}, x_{m}, x\right) & & \text { by definition of power triangle inequality at } p=\infty \\
& =2 \sigma \max \left\{\mathrm{d}\left(x_{n}, x\right), \mathrm{d}\left(x_{m}, x\right)\right\} & & \text { by Corollary } 4.7 \text { (page 17) } \\
& =2 \sigma \max \{\varepsilon, \varepsilon\} & & \text { by convergent hypothesis (Definition A.16 page 28) } \\
& =2 \sigma \varepsilon & & \text { by definition of max } \\
\mathrm{d}\left(x_{n}, x_{m}\right) & \leq \tau\left(-\infty, \sigma ; x_{n}, x_{m}, x\right) & & \text { by definition of power triangle inequality at } p=-\infty \\
& =2 \sigma \min \left\{\mathrm{d}\left(x_{n}, x\right), \mathrm{d}\left(x_{m}, x\right)\right\} & & \text { by Corollary 4.7 (page 17) } \\
& =2 \sigma \min \{\varepsilon, \varepsilon\} & & \text { by convergent hypothesis (Definition A.16 page 28) } \\
& =2 \sigma \varepsilon & & \text { by definition of min } \\
\mathrm{d}\left(x_{n}, x_{m}\right) & \leq \tau\left(0, \sigma ; x_{n}, x_{m}, x\right) & & \text { by definition of power triangle inequality at } p=0 \\
& =2 \sigma \sqrt{\mathrm{d}\left(x_{n}, x\right)} \sqrt{\mathrm{d}\left(x_{m}, x\right)} & & \text { by Corollary } 4.7 \text { (page 17) } \\
& =2 \sigma \sqrt{\varepsilon} \sqrt{\varepsilon} & & \text { by convergent hypothesis (Definition A.16 page 28) } \\
& =2 \sigma \varepsilon & & \text { by property of } \mathbb{R}
\end{aligned}
$$

(2) Proof that Cauchy $\Longrightarrow$ bounded: by Proposition 3.14 (page 9).

Theorem 4.17 ${ }^{45}$ Let $(X, \mathrm{~d}, p, \sigma)$ be a POWER Distance SPACE. Let $\mathrm{f} \in \mathbb{Z}^{\mathbb{Z}}$ be a STRICTLY MONOTONE function such that $\mathrm{f}(n)<\mathrm{f}(n+1)$. For any $(p, \sigma) \in \mathbb{R}^{*} \times \mathbb{R}^{+}$

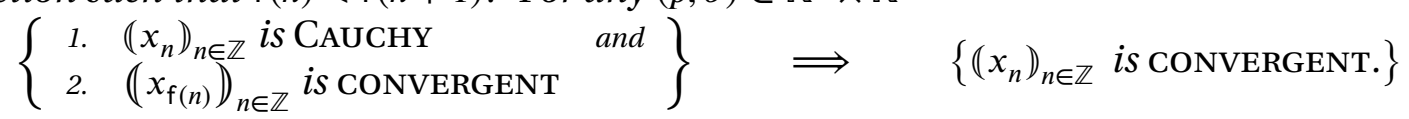

QPRoOF:

$$
\begin{aligned}
\mathrm{d}\left(x_{n}, x\right) & =\mathrm{d}\left(x, x_{n}\right) & & \text { by symmetric property of } \mathrm{d} \\
& \leq \tau\left(p, \sigma ; x, x_{n}, x_{\mathrm{f}(n)}\right) & & \text { by definition of power triangle inequality (Definition 4.3 page 16) } \\
& \triangleq 2 \sigma\left[\frac{1}{2} \mathrm{~d}^{p}\left(x, x_{\mathrm{f}(n)}\right)+\frac{1}{2} \mathrm{~d}^{p}\left(x_{\mathrm{f}(n)}, x_{n}\right)\right]^{\frac{1}{p}} & & \text { by definition of power triangle function (Definition 4.1 page 15) } \\
& =2 \sigma\left[\frac{1}{2} \varepsilon+\frac{1}{2} \mathrm{~d}^{p}\left(x_{\mathrm{f}(n)}, x_{n}\right)\right]^{\frac{1}{p}} & & \text { by left hypothesis } 2 \\
& =2 \sigma\left[\frac{1}{2} \varepsilon^{p}+\frac{1}{2} \varepsilon^{p}\right]^{\frac{1}{p}} & & \text { by left hypothesis } 1 \\
& =2 \sigma \varepsilon & & \\
& \Longrightarrow \text { convergent } & & \text { by definition of convergent (Definition A.16 page 28) }
\end{aligned}
$$

\footnotetext{
${ }^{45}$ in metric space: $\ominus[$ Rosenlicht(1968)] page 52
} 
Theorem 4.18 ${ }^{46}$ Let $(X, \mathrm{~d}, p, \sigma)$ be a POWER DISTANCE SPACE. Let $(\mathbb{R}, \mathrm{q})$ be a metric space of real numbers with the usual metric $\mathrm{q}(x, y) \triangleq|x-y|$. Then

$$
\left\{2 \sigma=2^{\frac{1}{p}}\right\} \quad \Longrightarrow \quad\{\mathrm{d} \text { is continuous in }(\mathbb{R}, \mathrm{q})\}
$$

QPROOF:

$$
\begin{aligned}
\left|\mathrm{d}(x, y)-\mathrm{d}\left(x_{n}, y_{n}\right)\right| & \leq\left|\mathrm{d}(x, y)-\mathrm{d}\left(x_{n}, y\right)\right|+\left|\mathrm{d}\left(x_{n}, y\right)-\mathrm{d}\left(x_{n}, y_{n}\right)\right| & & \text { by triangle inequality of }(\mathbb{R},|x-y|) \\
& =\left|\mathrm{d}(x, y)-\mathrm{d}\left(y, x_{n}\right)\right|+\left|\mathrm{d}\left(y, x_{n}\right)-\mathrm{d}\left(x_{n}, y_{n}\right)\right| & & \text { by commutative property of } \mathrm{d} \text { (Definition 3.1 page 6) } \\
& \leq \mathrm{d}\left(x, x_{n}\right)+\mathrm{d}\left(y, y_{n}\right) & & \text { by } 2 \sigma=2^{\frac{1}{p}} \text { and Lemma } 4.9 \text { (page 18) } \\
& =0 \quad \text { as } n \rightarrow \infty & &
\end{aligned}
$$

In distance spaces and topological spaces, limits of convergent sequences are in general not unique (Example 3.21 page 12, Example A.17 page 29). However Theorem 4.19 (next) demonstrates that, in a power distance space, limits are unique.

Theorem 4.19 (Uniqueness of limit) ${ }^{47}$ Let $(X, \mathrm{~d}, p, \sigma)$ be a POWER DistanCE SPACE. Let $x, y, \in X$ and let $\left(x_{n} \in X\right)$ be an $X$-valued sequence.

$$
\left\{\begin{array}{ll}
\text { 1. } & \left\{\left(\left(x_{n}\right),\left(y_{n}\right)\right) \rightarrow(x, y)\right\} \quad \text { and } \\
\text { 2. } & (p, \sigma) \in \mathbb{R}^{*} \times \mathbb{R}^{+}
\end{array}\right\} \Longrightarrow\{x=y\}
$$

PROOF:

(1) lemma: Proof that for all $(p, \sigma) \in \mathbb{R}^{*} \times \mathbb{R}^{+}$and for any $\varepsilon \in \mathbb{R}^{+}$, there exists $N$ such that $\mathrm{d}(x, y)<2 \sigma \varepsilon$ :

$$
\begin{aligned}
\mathrm{d}(x, y) & \leq \tau\left(p, \sigma ; x, y, x_{n}\right) & & \text { by definition of power triangle inequality (Definition 4.3 page 16) } \\
& \triangleq 2 \sigma\left[\frac{1}{2} \mathrm{~d}^{p}\left(x, x_{n}\right)+\frac{1}{2} \mathrm{~d}^{p}\left(x_{n}, y\right)\right]^{\frac{1}{p}} & & \text { by definition of power triangle function (Definition 4.1 page 15) } \\
& <2 \sigma\left[\frac{1}{2} \varepsilon^{p}+\frac{1}{2} \varepsilon^{p}\right]^{\frac{1}{p}} & & \text { by left hypothesis and for } p \in \mathbb{R}^{*} \backslash\{-\infty, 0, \infty\} \\
& =2 \sigma \varepsilon & & \\
\mathrm{d}(x, y) & \leq \tau\left(\infty, \sigma ; x, y, x_{n}\right) & & \text { by definition of power triangle inequality at } p=\infty \\
& =2 \sigma \max \left\{\mathrm{d}\left(x, x_{n}\right), \mathrm{d}\left(x_{n}, y\right)\right\} & & \text { by Corollary } 4.7 \text { (page 17) } \\
& <2 \sigma \varepsilon & & \text { by left hypothesis } \\
\mathrm{d}(x, y) & \leq \tau\left(-\infty, \sigma ; x, y, x_{n}\right) & & \text { by definition of power triangle inequality at } p=-\infty \\
& =2 \sigma \min \left\{\mathrm{d}\left(x, x_{n}\right), \mathrm{d}\left(x_{n}, y\right)\right\} & & \text { by Corollary } 4.7 \text { (page 17) } \\
& <2 \sigma \varepsilon & & \text { by left hypothesis } \\
\mathrm{d}(x, y) & \leq \tau\left(0, \sigma ; x, y, x_{n}\right) & & \text { by definition of power triangle inequality at } p=0 \\
& =2 \sigma \sqrt{\mathrm{d}\left(x, x_{n}\right)} \sqrt{\mathrm{d}\left(x_{n}, y\right)} & & \text { by Corollary } 4.7 \text { (page 17) } \\
& =2 \sigma \sqrt{\varepsilon} \sqrt{\varepsilon} & & \text { by left hypothesis } \\
& <2 \sigma \varepsilon & & \text { by property of real numbers }
\end{aligned}
$$

\footnotetext{
${ }^{46}$ in metric space $((p, \sigma)=(1,1)$ case $): \theta$ [Berberian(1961)] page $37\langle$ Theorem II.4.1〉

${ }^{47}$ in metric space: $\theta$ [Rosenlicht(1968)] page 46, $\theta$ [Thomson et al.(2008)Thomson, Bruckner, and Bruckner] page $32\langle$ Theorem 2.8 〉
} 
(2) Proof that $x=y$ (proof by contradiction):

$$
\begin{aligned}
x \neq y & \Longrightarrow \mathrm{d}(x, y) \neq 0 \\
& \Longrightarrow \mathrm{d}(x, y)>0 \\
& \Longrightarrow \exists \varepsilon \quad \text { such that } \mathrm{d}(x, y)>2 \sigma \varepsilon \\
& \Longrightarrow \text { contradiction to (1) lemma page } 23 \\
& \Longrightarrow \mathrm{d}(x, y)=0 \\
& \Longrightarrow x=y
\end{aligned}
$$

by the nondegenerate property of $d$ (Definition 3.1 page 6)

by non-negative property of $d$ (Definition 3.1 page 6)

\subsection{Examples}

It is not always possible to find a triangle relation (Definition 4.3 page 16) $\otimes(p, \sigma ; \mathrm{d}$ ) that holds in every distance space (Definition 3.1 page 6), as demonstrated by Example 4.20 and Example 4.21 (next two examples).

Example 4.20 Let $\mathrm{d}(x, y) \in \mathbb{R}^{\mathbb{R} \times \mathbb{R}}$ be defined such that

$$
\mathrm{d}(x, y) \triangleq\left\{\begin{array}{lll}
y & \forall(x, y) \in\{4\} \times(0: 2] & \text { (vertical half-open interval) } \\
x & \forall(x, y) \in(0: 2] \times\{4\} & \text { (horizontal half-open interval) } \\
|x-y| & \text { otherwise } & \text { (Euclidean) }
\end{array}\right\} .
$$

Note the following about the pair $(\mathbb{R}, d)$ :

(1) By Example 3.21 (page 12 ), $(\mathbb{R}, \mathrm{d})$ is a distance space, but not a metric space - that is, the triangle relation $\otimes(1,1 ; d)$ does not hold in $(\mathbb{R}, d)$.

(2) Observe further that $(\mathbb{R}, d)$ is not a power distance space. In particular, the triangle relation $\otimes(p, \sigma ; d)$ does not hold in $\left(\mathbb{R}, d\right.$ ) for any finite value of $\sigma$ (does not hold for any $\sigma \in \mathbb{R}^{+}$):

$$
\begin{aligned}
\mathrm{d}(0,4)=4 \not \leq 0=\lim _{\varepsilon \rightarrow 0} 2 \sigma \varepsilon & =\lim _{\varepsilon \rightarrow 0} 2 \sigma\left[1 / 2|0-\varepsilon|^{p}+1 / 2 \varepsilon^{p}\right]^{\frac{1}{p}} \\
& \triangleq \lim _{\varepsilon \rightarrow 0} 2 \sigma\left[1 / 2 \mathrm{~d}^{p}(0, \varepsilon)+1 / 2 \mathrm{~d}^{p}(\varepsilon, 4)\right]^{\frac{1}{p}} \triangleq \lim _{\varepsilon \rightarrow 0} \otimes(p, \sigma ; 0,4, \varepsilon ; \mathrm{d})
\end{aligned}
$$

Example 4.21 Let $\mathrm{d}(x, y) \in \mathbb{R}^{\mathbb{R} \times \mathbb{R}}$ be defined such that

$$
\mathrm{d}(x, y) \triangleq\left\{\begin{array}{lll}
|x-y| & \text { for } x=0 \text { or } y=0 \text { or } x=y & \text { (Euclidean }) \\
1 & \text { otherwise } & \text { (discrete })
\end{array}\right\} .
$$

Note the following about the pair $(\mathbb{R}, d)$ :

(1) By Example 3.22 (page 13), $(\mathbb{R}, d$ ) is a distance space, but not a metric space-that is, the triangle relation $\otimes(1,1 ; d)$ does not hold in $(\mathbb{R}, d)$.

(2) Observe further that $(\mathbb{R}, d)$ is not a power distance space-that is, the triangle relation $\otimes(p, \sigma ; d)$ does not hold in $(\mathbb{R}, d)$ for any value of $(p, \sigma) \in \mathbb{R}^{*} \times \mathbb{R}^{+}$.

(a) Proof that $\otimes(p, \sigma ; \mathrm{d})$ does not hold for any $(p, \sigma) \in\{\infty\} \times \mathbb{R}^{+}$:

$$
\begin{aligned}
& \lim _{n, m \rightarrow \infty} \mathrm{d}(1 / n, 1 / m) \triangleq 1 \not \leq 0=2 \sigma \max \{0,0\} \quad \text { by definition of } \mathrm{d} \\
& =2 \sigma \lim _{n, m \rightarrow \infty} \max \{\mathrm{d}(1 / n, 0), \mathrm{d}(0,1 / m)\} \quad \text { by Corollary } 4.7 \text { (page 17) } \\
& \geq \lim _{n, m \rightarrow \infty} 2 \sigma\left[1 / 2 d^{p}(1 / n, 0)+1 / 2 d^{p}(0,1 / m)\right]^{\frac{1}{p}} \quad \text { by Corollary } 4.6 \text { (page 16) } \\
& \triangleq \lim _{n, m \rightarrow \infty} \tau(p, \sigma, 1 / n, 1 / m, 0) \quad \text { by definition of } \tau \text { (Definition } 4.1 \text { page 15) }
\end{aligned}
$$


(b) Proof that $\otimes(p, \sigma ; \mathrm{d})$ does not hold for any $(p, \sigma) \in \mathbb{R}^{*} \times \mathbb{R}^{+}$: By Corollary 4.6 (page 16), the triangle function (Definition 4.1 page 15) $\tau(p, \sigma ; x, y, z ; \mathrm{d})$ is continuous and strictly monotone in $(\mathbb{R},|\cdot|, \leq)$ with respect to the variable $p$. Item 2 a demonstrates that $\otimes(p, \sigma ; d)$ fails to hold at the best case of $p=\infty$, and so by Corollary 4.6, it doesn't hold for any other value of $p \in \mathbb{R}^{*}$ either.

Example 4.22 Let $d$ be a function in $\mathbb{R}^{\mathbb{R} \times \mathbb{R}}$ such that $\mathrm{d}(x, y) \triangleq\left\{\begin{array}{rll}2|x-y| & \forall(x, y) \in\{(0,1),(1,0)\} & \text { (dilated Euclidean) } \\ |x-y| & \text { otherwise } & \text { (Euclidean) }\end{array}\right\}$.

Note the following about the pair $(\mathbb{R}, d)$ :

(1) By Example 3.23 (page 14$),(\mathbb{R}, d)$ is a distance space, but not a metric space-that is, the triangle relation $\otimes(1,1 ; \mathrm{d})$ does not hold in $(\mathbb{R}, \mathrm{d})$.

(2) But observe further that $(\mathbb{R}, \mathrm{d}, 1,2)$ is a power distance space:

(a) Proof that $\otimes(1,2 ; \mathrm{d})$ (Definition 4.3 page 16) holds for all $(x, y) \in\{(0,1),(1,0)\}$ :

$$
\begin{aligned}
\mathrm{d}(1,0) & =\mathrm{d}(0,1) \triangleq 2|0-1|=2 & & \text { by definition of } \mathrm{d} \\
& \leq 2 \leq 2(|0-z|+|z-1|) \quad \forall z \in \mathbb{R} & & \text { by definition of }|\cdot| \text { (Definition 2.10 page 5) } \\
& =2 \sigma\left(1 / 2|0-z|^{p}+1 / 2|z-1|^{p}\right)^{\frac{1}{p}} \quad \forall z \in \mathbb{R} & & \text { for }(p, \sigma)=(1,2) \\
& \triangleq 2 \sigma\left(1 / 2 \mathrm{~d}^{p}(0, z)+\mathrm{d}^{p}(z, 1)\right)^{\frac{1}{p}} \quad \forall z \in \mathbb{R} & & \text { for }(p, \sigma)=(1,2) \text { and by definition of } \mathrm{d} \\
& \triangleq \tau(1,2 ; 0,1, z) & & \text { by definition of } \tau \text { (Definition 4.1 page 15) }
\end{aligned}
$$

(b) Proof that $\otimes(1,2 ; \mathrm{d})$ holds for all other $(x, y) \in \mathbb{R}^{*} \times \mathbb{R}^{+}$:

$$
\begin{aligned}
\mathrm{d}(x, y) & \triangleq 2|x-y| & & \text { by definition of } \mathrm{d} \\
& \leq(|x-z|+|z-y|) & & \text { by property of Euclidean metric spaces } \\
& =2 \sigma\left(1 / 2|0-z|^{p}+1 / 2|z-1|^{p}\right)^{\frac{1}{p}} & & \text { for }(p, \sigma)=(1,1) \\
& \triangleq \tau(1,1 ; x, y, z) & & \text { by definition of } \tau \text { (Definition 4.1 page 15) } \\
& \leq \tau(1,2 ; x, y, z) & & \text { by Corollary 4.6 (page 16) }
\end{aligned}
$$

(3) In $(X, d)$, the limits of convergent sequences are unique. This follows directly from the fact that $(\mathbb{R}, d, 1,2)$ is a power distance space (item (2) page 25) and by Theorem 4.19 page 23.

(4) In $(X, d)$, convergent sequences are Cauchy. This follows directly from the fact that $(\mathbb{R}, \mathrm{d}, 1,2)$ is a power distance space (item (2) page 25) and by Theorem 4.16 page 21.

Example 4.23 Let $\mathrm{d}$ be a function in $\mathbb{R}^{\mathbb{R} \times \mathbb{R}}$ such that $\mathrm{d}(x, y) \triangleq(x-y)^{2}$. Note the following about the pair $(\mathbb{R}, d)$ :

(1) It was demonstrated in Example 3.24 (page 15$)$ that $(\mathbb{R}, d)$ is a distance space, but that it is not a metric space because the triangle inequality does not hold.

(2) However, the tuple $(\mathbb{R}, \mathrm{d}, p, \sigma)$ is a power distance space (Definition 4.3 page 16 ) for any $(p, \sigma) \in \mathbb{R}^{*} \times$ $[2: \infty)$ : In particular, for all $x, y, z \in \mathbb{R}$, the power triangle inequality (Definition 4.3 page 16) must hold. The "worst case" for this is when a third point $z$ is exactly "halfway between" $x$ and $y$ in $\mathrm{d}(x, y)$; 
that is, when $z=\frac{x+y}{2}$ :

$$
\begin{array}{rlrl}
(x-y)^{2} & \triangleq \mathrm{d}(x, y) & & \text { by definition of } \mathrm{d} \\
& \leq \tau(p, \sigma ; x, y, z ; \mathrm{d}) & & \text { by definition power triangle inequality } \\
& \triangleq 2 \sigma\left[1 / 2 \mathrm{~d}^{p}(x, z)+{ }^{1 / 2} \mathrm{~d}^{p}(z, y)\right]^{\frac{1}{p}} & & \text { by definition } \tau \text { (Definition 4.1 page 15) } \\
& \triangleq 2 \sigma\left[1 / 2(x-z)^{2 p}+1 / 2(z-y)^{2 p}\right]^{\frac{1}{p}} & & \text { by definition of } \mathrm{d} \\
& =2 \sigma\left[1 / 2|x-z|^{2 p}+1 / 2|z-y|^{2 p}\right]^{\frac{1}{p}} & & \text { because }(x)^{2}=|x|^{2} \text { for all } x \in \mathbb{R} \\
& =2 \sigma\left[1 / 2\left|x-\frac{x+y}{2}\right|^{2 p}+1 / 2\left|\frac{x+y}{2}-y\right|^{2 p}\right]^{\frac{1}{p}} & & \text { because } z=\frac{x+y}{2} \text { is the "worst case" scenario } \\
& =2 \sigma\left[1 / 2\left|\frac{y-x}{2}\right|^{2 p}+1 / 2\left|\frac{x-y}{2}\right|^{2 p}\right]^{\frac{1}{p}} & \\
& =2 \sigma\left[\left|\frac{x-y}{2}\right|^{2 p}\right]^{\frac{1}{p}}=\frac{2 \sigma}{4}|x-y|^{2} & \\
& \Longrightarrow(p, \sigma) \in \mathbb{R}^{*} \times[2: \infty) &
\end{array}
$$

(3) The power distance function $\mathrm{d}$ is continuous in $(\mathbb{R}, \mathrm{d}, p, \sigma)$ for any $(p, \sigma)$ such that $\sigma \geq 2$ and $2 \sigma=$ $p^{\frac{1}{p}}$. This follows directly from Theorem 4.18 (page 23).

\section{Appendix A Topological Spaces}

Definition A.1 ${ }^{48}$ Let $\Gamma$ be a set with an arbitrary (possibly uncountable) number of elements. Let $2^{X}$ be the power set of a set $X$ (Definition 2.2 page 4). A family of sets $T \subseteq 2^{X}$ is a topology on $X$ if

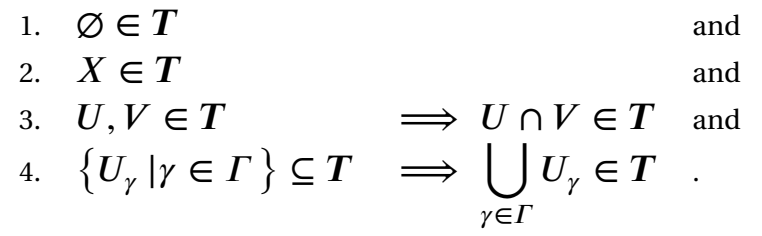

The ordered pair $(X, \boldsymbol{T})$ is a topological space if $\boldsymbol{T}$ is a topology on $X$. A set $U$ is open in $(X, T)$ if $U$ is any element of $\boldsymbol{T}$. A set $D$ is closed in $(X, \boldsymbol{T})$ if $D^{\mathrm{c}}$ is open in $(X, \boldsymbol{T})$.

Just as the power set $2^{X}$ and the set $\{\varnothing, X\}$ are algebras of sets on a set $X$, so also are these sets topologies on $X$ (next example):

Example A.2 ${ }^{49}$ Let $\mathcal{T}(X)$ be the set of topologies on a set $X$ and $2^{X}$ the power set (Definition 2.2 page 4) on $X$.

$\{\varnothing, X\}$ is a topology in $\mathcal{T}(X) \quad$ (indiscrete topology or trivial topology)

$2^{X} \quad$ is a topology in $\mathcal{T}(X) \quad$ (discrete topology)

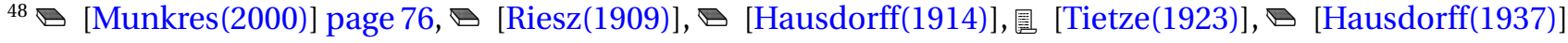
page 258

${ }_{49} \otimes[$ Munkres(2000)], page 77, $\otimes[$ [Kubrusly(2011)] page $107\langle$ Example 3.J $\rangle, \otimes$ [Steen and Seebach(1978)] pages $42-43\langle$ II.4 $\rangle, \boxminus$ [DiBenedetto(2002)] page 18
} 
Definition A.3 ${ }^{50}$ Let $(X, \boldsymbol{T})$ be a topological space. A set $\boldsymbol{B} \subseteq 2^{X}$ is a base for $\boldsymbol{T}$ if

1. $\boldsymbol{B} \subseteq \boldsymbol{T}$ and

2. $\forall U \in \boldsymbol{T}, \quad \exists\left\{\boldsymbol{B}_{\gamma} \in \boldsymbol{B}\right\} \quad$ such that $\quad U=\bigcup_{\gamma} B_{\gamma}$

Theorem A.4 ${ }^{51}$ Let $(X, T)$ be a TOPOLOGICAL SPACE. Let $\boldsymbol{B}$ be a subset of $2^{X}$ such that $\boldsymbol{B} \subseteq 2^{X}$. $\{\boldsymbol{B}$ is $a$ BASE for $\boldsymbol{T}\} \quad\left\{\begin{array}{l}\text { For every } x \in X \text { and for every OPEN SET } U \text { containing } x, \\ \text { there exists } B_{x} \in \boldsymbol{B} \text { such that } \quad x \in B_{x} \subseteq U .\end{array}\right\}$

Theorem A.5 ${ }^{52} \operatorname{Let}(X, \boldsymbol{T})$ be a TOPOLOGICAL SPACE (Definition A.1 page 26) and $\boldsymbol{B} \subseteq 2^{X}$.

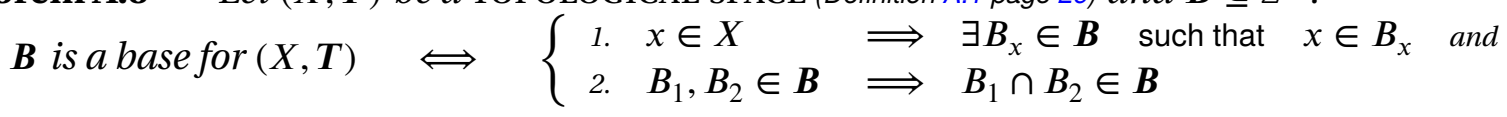

Example A.6 ${ }^{53}$ Let $(X, \mathrm{~d})$ be a metric space. The set $\boldsymbol{B} \triangleq\{\mathrm{B}(x, r) \mid x \in X, r \in \mathbb{N}\}$ (the set of all open balls in $(X, \mathrm{~d}))$ is a base for a topology on $(X, \mathrm{~d})$.

Example A.7 (the standard topology on the real line) ${ }^{54}$ The set $\boldsymbol{B} \triangleq\{(a: b) \mid a, b \in \mathbb{R}, a<b\}$ is a base for the metric space $(\mathbb{R},|b-a|)$ (the usual metric space on $\mathbb{R}$ ).

Definition A.8 ${ }^{55}$ Let $(X, T)$ be a topological space (Definition A.1 page 26). Let $2^{X}$ be the power set of $X$.

The set $A^{-}$is the closure of $A \in 2^{X}$ if $A^{-} \triangleq \bigcap\left\{D \in 2^{X} \mid A \subseteq D\right.$ and $D$ is closed $\}$.

The set $A^{\circ}$ is the interior of $A \in 2^{X}$ if $\quad A^{\circ} \triangleq \bigcup\left\{U \in 2^{X} \mid U \subseteq A\right.$ and $U$ is open $\}$.

A point $x$ is a closure point of $A$ if $x \in A^{-}$.

A point $x$ is an interior point of $A$ if $x \in A^{\circ}$.

A point $x$ is an accumulation point of $A$ if $x \in(A \backslash\{x\})^{-}$

A point $x$ in $A^{-}$is a point of adherence in $A$ or is adherent to $A$ if $x \in A^{-}$.

Proposition A.9 ${ }^{56}$ Let $(X, T)$ be a TOPOLOGICAL SPACE (Definition A.1 page 26). Let $A^{-}$be the ClOsURE, $A^{\circ}$ the INTERIOR, and $\partial A$ the BOUNDARY of a set $A$. Let $2^{X}$ be the POWER SET of $X$.

1. $A^{-}$is CLOSED $\forall A \in 2^{X}$.

2. $A^{\circ}$ is OPEN $\forall A \in \mathbb{2}^{X}$.

Lemma A.10 ${ }^{57}$ Let $A^{-}$be the CLOSURE, $A^{\circ}$ the INTERIOR, and $\partial A$ the BOUNDARY of a set $A$ in a topological space $(X, T)$. Let $2^{X}$ be the POWER SET of $X$.
1. $A^{\circ} \subseteq A \subseteq A^{-}$
$\forall A \in 2^{X}$.
2. $A=A^{\circ} \Longleftrightarrow A$ is OPEN $\forall A \in 2^{X}$.
3. $A=A^{-} \Longleftrightarrow A$ is CLOSED $\forall A \in \mathbb{2}^{X}$.

\footnotetext{
${ }^{50} \boxminus[$ Joshi(1983)] page $92\langle(3.1)$ Definition $\rangle, \theta$ [Davis(2005)] page $46\langle$ Definition 4.15$\rangle$

${ }^{51} \boxminus[$ Joshi(1983)] pages 92-93<(3.2) Proposition $\rangle, \otimes[$ Davis(2005)] page 46

$52 \boxminus$ [Bollobás(1999)] page 19

${ }^{53} \boxminus$ [Davis(2005)] page $46\langle$ Example 4.16〉

${ }^{54} \boxminus$ [Munkres(2000)] page 81, $\boxminus$ [Davis(2005)] page $46\langle$ Example 4.16〉

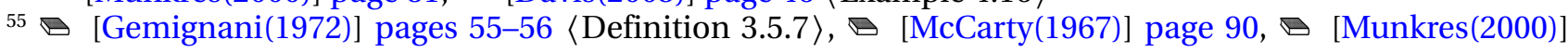

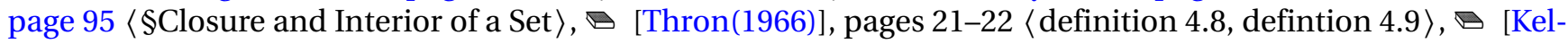
ley(1955)] page 42, $\bullet$ [Kubrusly(2001)] pages 115-116

$56 \otimes[$ McCarty(1967)] page $90\langle$ IV.1 THEOREM $\rangle$

$57 \boxminus[$ McCarty(1967)] pages 90-91 〈IV.1 THEOREM $\rangle, \otimes[$ ALIPRANTIS AND BuRKINSHaW(1998)] PAGE 59
} 
Definition A.11 ${ }^{58}$ Let $\left(X, T_{x}\right)$ and $\left(Y, T_{y}\right)$ be topological spaces (Definition A.1 page 26). Let $\mathrm{f}$ be a function in $Y^{X}$. A function $f \in Y^{X}$ is continuous if $\quad \underbrace{U \in \boldsymbol{T}_{y}}_{\text {open in }\left(Y, \boldsymbol{T}_{y}\right)} \Longrightarrow \underbrace{\mathrm{f}^{-1}(\boldsymbol{U}) \in \boldsymbol{T}_{x}}_{\text {open } \text { in }\left(X, \boldsymbol{T}_{x}\right)}$.

A function is discontinuous in $\left(X, \boldsymbol{T}_{y}\right)^{\left(X, \boldsymbol{T}_{x}\right)}$ if it is not continuous in $\left(X, \boldsymbol{T}_{y}\right)^{\left(X, \boldsymbol{T}_{x}\right)}$.

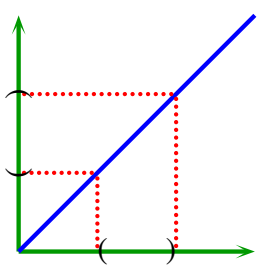

continuous

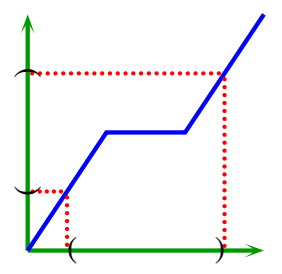

continuous

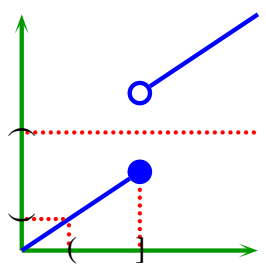

discontinuous

Figure 3: continuous/ discontinuous functions (Example A.12 page 28)

Example A.12 Some continuous/discontinuous functions are illustrated in Figure 3 (page 28).

Definition A.11 (previous definition) defines continuity using open sets. Continuity can alternatively be defined using closed sets or closure (next theorem).

Theorem A.13 ${ }^{59}$ Let $(X, T)$ and $(Y, S)$ be topological spaces. Let $\mathrm{f}$ be a function in $Y^{X}$. The following are equivalent:

$\begin{array}{lll}\text { 1. } \mathrm{f} \text { is continuous } & & \Longleftrightarrow \\ \text { 2. } \quad B \text { is closed in }(Y, S) \Longrightarrow \mathrm{f}^{-1}(B) \text { is closed in }(X, T) & \forall B \in 2^{Y} & \Longleftrightarrow \\ \text { 3. } \quad \mathrm{f}\left(A^{-}\right) \subseteq \mathrm{f}(A)^{-} & \\ \text {4. } \mathrm{f}^{-1}(B)^{-} \subseteq \mathrm{f}^{X} & \Longleftrightarrow \\ & \forall B \in \mathbb{2}^{-1}\left(B^{-}\right) & \end{array}$

Remark A.14 A word of warning about defining continuity in terms of topological spaces-continuity is defined in terms of a pair of topological spaces, and whether function is continuous or discontinuous in general depends very heavily on the selection of these spaces. This is illustrated in Proposition A.15 (next). The ramification of this is that when declaring a function to be continuous or discontinuous, one must make clear the assumed topological spaces.

Proposition A.15 ${ }^{60} \operatorname{Let}(X, T)$ and $(Y, S)$ be topological spaCes. Let $\mathrm{f}$ be a FunCtion in $(Y, S)^{(X, T)}$.

1. $T$ is the DISCRETE TOPOLOGY $\Longrightarrow \mathrm{f}$ is CONTINUOUS $\forall \mathrm{f} \in(Y, S)^{(X, T)}$

2. $S$ is the INDISCRETE TOPOLOGY $\Longrightarrow \mathrm{f}$ is CONTINUOUS $\forall \mathrm{f} \in(Y, S)^{(X, T)}$

Definition A.16 ${ }^{61}$ Let $(X, T)$ be a topological space (Definition A.1 page 26). A sequence $\left(x_{n}\right)_{n \in \mathbb{Z}}$ converges in $(X, \boldsymbol{T})$ to a point $x$ if for each open set (Definition A.1 page 26) $U \in \boldsymbol{T}$ that contains $x$ there exists $N \in \mathbb{N}$ such that

\footnotetext{
${ }^{58} \boxminus$ [Davis(2005)] page 34

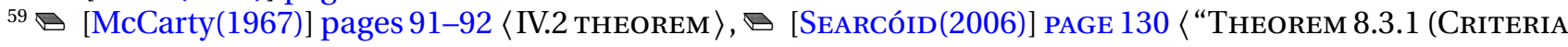
FOR CONTINUITY)", SET IN metric spaces $\rangle$

$60 \triangleq[$ Crossley(2006)] page $18\langle$ Proposition 3.9〉, $\boxminus$ [Ponnusamy(2002)] page $98\langle 2.64$. Theorem. $\rangle$

${ }^{61} \boxminus[$ Joshi(1983)] page $83\langle(3.1)$ Definition〉, $\boxminus$ [Leathem(1905)], page $13\langle “ \rightarrow$ " symbol, section III.11〉
} 
$x_{n} \in U$ for all $n>N$.

This condition can be expressed in any of the following forms:

1. The limit of the sequence $\left(x_{n}\right)$ is $x . \quad 3 . \lim _{n \rightarrow \infty}\left(x_{n}\right)=x$.

2. The sequence $\left(x_{n}\right)$ is convergent with limit $x . \quad$ 4. $\quad\left(x_{n}\right) \rightarrow x$.

A sequence that converges is convergent. A sequence that does not converge is said to diverge, or is divergent. An element $x \in A$ is a limit point of $A$ if it is the limit of some $A$-valued sequence $\left(x_{n} \in A\right)$.

Example A.17 ${ }^{62}$ Let $\left(X, \boldsymbol{T}_{31}\right)$ be a topological space where $X \triangleq\{x, y, z\}$ and $\boldsymbol{T}_{31} \triangleq\{\varnothing,\{x\},\{x, y\},\{x, z\},\{x, y, z\}\}$.

In this space, the sequence $(x, x, x, \ldots)$ converges to $x$. But this sequence also converges to both $y$ and $z$ because $x$ is in every open set (Definition A.1 page 26) that contains $y$ and $x$ is in every open set that contains $z$. So, the limit (Definition A.16 page 28) of the sequence is not unique.

Example A.18 In contrast to the low resolution topological space of Example A.17, the limit of the sequence $(x, x, x, \ldots)$ is unique in a topological space with sufficiently high resolution with respect to $y$ and $z$ such as the following: Define a topological space $\left(X, \boldsymbol{T}_{56}\right)$ where $X \triangleq\{x, y, z\}$ and

\section{$\boldsymbol{T}_{56} \triangleq\{\varnothing,\{y\},\{z\},\{x, y\},\{y, z\},\{x, y, z\}\}$.}

In this space, the sequence $(x, x, x, \ldots)$ converges to $x$ only. The sequence does not converge to $y$ or $z$ because there are open sets (Definition A.1 page 26) containing $y$ or $z$ that do not contain $x$ (the open sets $\{y\},\{z\}$, and $\{y, z\})$.

Theorem A.19 (The Closed Set Theorem) ${ }^{63}$ Let $(X, T)$ be a TOPOLOGICAL SPACE. Let $A$ be a subset of $X(A \subseteq X)$. Let $A^{-}$be the Closure (Definition A.8 page 27) of $A$ in $(X, T)$.

$$
\underbrace{A \text { is CLOSED in }(X, T)}_{\left(A=A^{-}\right)} \Longleftrightarrow \quad\left\{\begin{array}{l}
\text { Every A-valued sequence }\left(x_{n} \in A\right)_{n \in \mathbb{Z}} \\
\text { that CONVERES in }(X, T) \text { has its LIMIT in } A
\end{array}\right\}
$$

Theorem A.20 ${ }^{64} \operatorname{Let}(X, \boldsymbol{T})$ and $(Y, S)$ be a TOPOLOGICAL SPACES. Let $\mathrm{f}$ be a function in $(Y, S)^{(X, T)}$.

$$
\underbrace{\left\{\begin{array}{c}
\mathrm{f} \text { is CONTINUOUs } \operatorname{in}(Y, S)^{(X, T)} \\
\text { (Definition A.11 page 28) }
\end{array}\right\}}_{\text {INVERSE IMAGE CHARACTERIZATION OF CONTINUITY }} \Longleftrightarrow \underbrace{\left\{\begin{array}{c}
\left(x_{n}\right) \rightarrow \begin{array}{c}
\rightarrow \\
\text { (Definition A.16 page 28) }
\end{array} \\
\mathrm{f}\left(\left(x_{n}\right)\right) \rightarrow \mathrm{f}(x)
\end{array}\right\}}_{\text {SEQUENTIAL CHARACTERIZATION OF CONTINUITY }}
$$

QPRoOF:

(1) Proof for the $\Longrightarrow$ case (proof by contradiction):

(a) Let $U$ be an open set in $(Y, T)$ that contains $f(x)$ but for which there exists no $N$ such that $f\left(x_{n}\right) \in U$ for all $n>N$.

(b) Note that the set $\mathrm{f}^{-1}(U)$ is also open by the continuity hypothesis.

\footnotetext{
$62 \boxminus[$ Munkres(2000)] page 98 〈Hausdorff Spaces〉

${ }^{63} \boxminus[$ Kubrusly(2001)] page $118\langle$ Theorem 3.30〉, $\boxminus$ [Haaser and Sullivan(1991)] page $75\langle 6 \cdot 9$ Proposition $\rangle$, $\boxminus[$ Rosenlicht(1968)] pages 47-48

$64 \boxminus$ [Ponnusamy(2002)] pages 94-96 〈"2.59. Proposition.”; in the context of metric spaces; includes the "inverse image characterization of continuity" and "sequential characterization of continuity" terminology; this terminology does not seem to be widely used in the literature in general, but has been adopted for use in this text $\rangle$
} 
(c) If $\left(x_{n}\right) \rightarrow x$, then

$$
\begin{aligned}
\mathrm{f}\left(\left(x_{n}\right)\right) \nrightarrow \mathrm{f}(x) & \Longrightarrow \text { there exists no } N \text { such that } \mathrm{f}\left(x_{n}\right) \in U \text { for all } n>N \quad \text { by Definition A.16 (page 28) } \\
& \Longrightarrow \text { there exists no } M \text { such that } x_{n} \in \mathrm{f}^{-1}(U) \text { for all } n>M \quad \text { by definition of } \mathrm{f}^{-1} \\
& \Longrightarrow\left(x_{n}\right) \nrightarrow x \quad \text { by continuity hypothesis and def. of convergence (Definition A.16 page 28) } \\
& \Longrightarrow \text { contradiction of }\left(x_{n}\right) \rightarrow x \text { hypothesis } \\
& \Longrightarrow \mathrm{f}\left(\left(x_{n}\right)\right) \rightarrow \mathrm{f}(x)
\end{aligned}
$$

(2) Proof for the $\Longleftarrow$ case (proof by contradiction):

(a) Let $D$ be a closed set in $(Y, S)$.

(b) Suppose $\mathrm{f}^{-1}(D)$ is not closed...

(c) then by the closed set theorem (Theorem A.19 page 29), there must exist a convergent sequence $\left(x_{n}\right)$ in $(X, \boldsymbol{T})$, but with limit $x$ not in $\mathrm{f}^{-1}(D)$.

(d) Note that $\mathrm{f}(x)$ must be in $D$. Proof:

(i) by definition of $D$ and $f, f\left(\left(x_{n}\right)\right)$ is in $D$

(ii) by left hypothesis, the sequence $\mathrm{f}\left(\left(x_{n}\right)\right)$ is convergent with limit $\mathrm{f}(x)$

(iii) by closed set theorem (Theorem A.19 page 29), $\mathrm{f}(x)$ must be in $D$.

(e) Because $\mathrm{f}(x) \in D$, it must be true that $x \in \mathrm{f}^{-1}(D)$.

(f) But this is a contradiction to item (2c) (page 30), and so item (2b) (page 30) must be wrong, and $\mathrm{f}^{-1}(D)$ must be closed.

(g) And so by Theorem A.13 (page 28), $f$ is continuous.

\section{Appendix B Finite sums}

\section{B.1 Convexity}

\section{Definition B.1 65}

A function $f \in \mathbb{R}^{\mathbb{R}}$ is convex if

$$
f(\lambda x+[1-\lambda] y) \leq \lambda f(x)+(1-\lambda) f(y) \quad \forall x, y \in \mathbb{R} \text { and } \forall \lambda \in(0: 1)
$$

A function $g \in \mathbb{R}^{\mathbb{R}}$ is strictly convex if

$$
\mathrm{g}(\lambda x+[1-\lambda] y)=\lambda \mathrm{g}(x)+(1-\lambda) \mathrm{g}(y) \quad \forall x, y \in D, x \neq y \text {, and } \forall \lambda \in(0: 1)
$$

A function $f \in \mathbb{R}^{\mathbb{R}}$ is concave if $-f$ is convex.

A function $f \in \mathbb{R}^{\mathbb{R}}$ is affine if $f$ is convex and concave.

Theorem B.2 (Jensen's Inequality) ${ }^{66}$ Let $f \in \mathbb{R}^{\mathbb{R}}$ be a function.

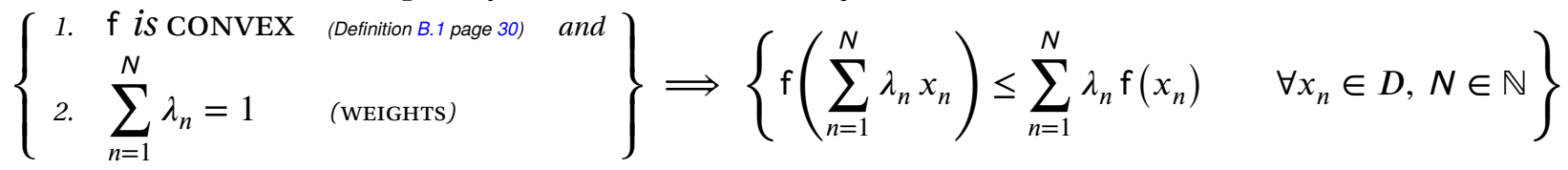

${ }_{65} \otimes[\operatorname{Simon}(2011)]$ page 2, $\theta[\operatorname{Barvinok}(2002)]$ page 2, $\otimes$ [Bollobás(1999)], page 3, $\theta$ [Jensen(1906)], page 176

${ }_{66} \boxminus$ [Mitrinović et al.(2010)Mitrinović, Pečarić, and Fink] page 6, $\oslash$ [Bollobás(1999)] page 3, 圈 [Jensen(1906)] pages $179-180$ 


\section{B.2 Power means}

\section{Definition B.3 67}

The $\left(\lambda_{n} D_{1}^{N}\right.$ weighted $\phi$-mean of a tuple $\left(x_{n}\right)_{1}^{N}$ is defined as

$$
\mathrm{M}_{\phi}\left(\left(x_{n} D\right) \triangleq \phi^{-1}\left(\sum_{n=1}^{N} \lambda_{n} \phi\left(x_{n}\right)\right)\right.
$$

where $\phi$ is a continuous and strictly monotonic function in $\mathbb{R}^{\mathbb{R}^{\vdash}}$

and $\left(\lambda_{n}\right)_{n=1}^{N}$ is a sequence of weights for which $\sum_{n=1}^{N} \lambda_{n}=1$.

Lemma B.4 ${ }^{68}$ Let $\mathrm{M}_{\phi}\left(\mid x_{n} D\right)$ be the $\left\langle\lambda_{n} D_{1}^{N}\right.$ weighted $\phi$-mean and $\mathrm{M}_{\psi}\left(\left|x_{n}\right\rangle\right)$ the $\left(\lambda \lambda_{n} D_{1}^{N}\right.$ weighted $\psi$ mean of a tuple $\left(\left.x_{n}\right|_{1} ^{N}\right.$.

$\phi \psi^{-1}$ is CONVEX and $\phi$ is INCREASING $\left.\Longrightarrow \mathrm{M}_{\phi}\left(\mid 0 x_{n} D\right) \geq \mathrm{M}_{\psi}\left(0 x_{n}\right\rangle\right)$

$\phi \psi^{-1}$ is CONVEX and $\phi$ is DECREASING $\left.\left.\Longrightarrow \mathrm{M}_{\phi}\left(0 x_{n}\right\rangle\right) \leq \mathrm{M}_{\psi}\left(0 x_{n}\right\rangle\right)$

$\phi \psi^{-1}$ is CONCAVE and $\phi$ is INCREASING $\Longrightarrow \mathrm{M}_{\phi}\left(0 x_{n} D\right) \leq \mathrm{M}_{\psi}\left(0 x_{n} D\right)$

$\phi \psi^{-1}$ is CONCAVE and $\phi$ is DECREASING $\Longrightarrow \mathrm{M}_{\phi}\left(0 x_{n} D\right) \geq \mathrm{M}_{\psi}\left(0 x_{n} D\right)$

One of the most well known inequalities in mathematics is Minkowski's Inequality. In 1946, H.P. Mulholland submitted a result that generalizes Minkowski's Inequality to an equal weighted $\phi$-mean. ${ }^{69}$ And Milovanović and Milovanovć (1979) generalized this even further to a weighted $\phi$-mean (next).

Theorem B.5 ${ }^{70}$ Let $\phi$ be a function in $\mathbb{R}^{\mathbb{R}}$.

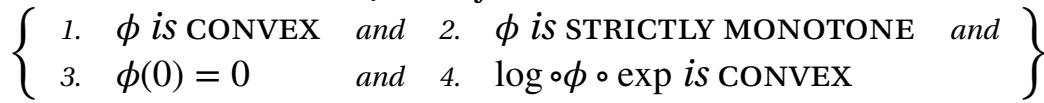

$$
\Longrightarrow\left\{\phi^{-1}\left(\sum_{n=1}^{N} \lambda_{n} \phi\left(x_{n}+y_{n}\right)\right) \leq \phi^{-1}\left(\sum_{n=1}^{N} \lambda_{n} \phi\left(x_{n}\right)\right)+\phi^{-1}\left(\sum_{n=1}^{N} \lambda_{n} \phi\left(y_{n}\right)\right)\right\}
$$

Definition B.6 ${ }^{71}$ Let $\mathrm{M}_{\phi(x ; p)}\left(\mid x_{n} D\right)$ be the $\left\langle\lambda_{n} D_{1}^{N}\right.$ weighted $\phi$-mean of a non-negative tuple $\left(x_{n} D_{1}^{N}\right.$. A mean $\mathrm{M}_{\phi(x ; p)}\left(0 x_{n} D\right)$ is a power mean with parameter $p$ if $\phi(x) \triangleq x^{p}$. That is,

$$
\mathrm{M}_{\phi(x ; p)}\left(\left(x_{n} D\right)=\left(\sum_{n=1}^{N} \lambda_{n}\left(x_{n}\right)^{p}\right)^{\frac{1}{p}}\right.
$$

Theorem B.7 ${ }^{72}$ Let $\mathrm{M}_{\phi(x ; p)}\left(\mid x_{n} D\right)$ be the POWER MEAN with parameter $p$ of an $N$-tuple $\left(x_{n} D_{1}^{N}\right.$ in which the elements are NOT all equal.

${ }^{67} \boxminus$ [Bollobás(1999)] page 5

$68 \boxminus$ [Pečarić et al.(1992)Pečarić, Proschan, and Tong] page 107, $\boxminus$ [Bollobás(1999)] page $5, \otimes$ [Hardy et al.(1952)Hardy, Littlewood, and Pólya] page 75

69 圈 [Minkowski(1910)] page 115, 圈 [Mulholland(1950)], $₫$ [Hardy et al.(1952)Hardy, Littlewood, and Pólya] $\langle$ Theorem 24〉, 圈 [Tolsted(1964)] page 7, 圈 [Maligranda(1995)] page 258, $\bullet$ [Carothers(2000)], page 44, $\boxminus$ [Bullen(2003)] page 179

70 圈 [Milovanović and Milovanović(1979)], $\bullet$ [Bullen(2003)] page $306\langle$ Theorem 9〉

${ }^{71} \boxminus$ [Bullen(2003)] page 175, $\boxminus$ [Bollobás(1999)] page 6

$72 \boxminus$ [Bullen(2003)] pages 175-177〈see also page 203〉, $\theta$ [Bollobás(1999)] pages 6-8, 圈 [Bullen(1990)] page 250, 圈 [Besso(1879)], 圈 [Bienaymé(1840)] page 68, 圈 [Brenner(1985)] page 160 


$$
\begin{aligned}
& \mathrm{M}_{\phi(x ; p)}\left(0 x_{n} D\right) \triangleq\left(\sum_{n=1}^{N} \lambda_{n}\left(x_{n}\right)^{p}\right)^{\frac{1}{p}} \text { is CONTINUOUs and STRICTLY MONOTONE in } \mathbb{R}^{*} \\
& \mathrm{M}_{\phi(x ; p)}\left(\left|x_{n}\right\rangle\right)= \begin{cases}\max _{n=1,2, \ldots, N}\left(x_{n}\right) & \text { for } p=+\infty \\
\prod_{n=1}^{N} x_{n}^{\lambda_{n}} & \text { for } p=0 \\
\min _{n=1,2, \ldots, N}\left(x_{n}\right) & \text { for } p=-\infty\end{cases}
\end{aligned}
$$

QPROOF:

(1) Proof that $M_{\phi(x ; p)}$ is strictly monotone in $p$ :

(a) Let $p$ and $s$ be such that $-\infty<p<s<\infty$.

(b) Let $\phi_{p} \triangleq x^{p}$ and $\phi_{s} \triangleq x^{s}$. Then $\phi_{p} \phi_{s}^{-1}=x^{\frac{p}{s}}$.

(c) The composite function $\phi_{p} \phi_{s}^{-1}$ is convex or concave depending on the values of $p$ and $s$ :

\begin{tabular}{c||c|c} 
& $p<0\left(\phi_{p}\right.$ decreasing $)$ & $p>0\left(\phi_{p}\right.$ increasing) \\
\hline \hline$s<0$ & convex & (not possible) \\
\hline$s>0$ & convex & concave \\
\hline
\end{tabular}

(d) Therefore by Lemma B.4 (page 31),

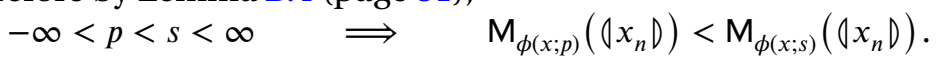

(2) Proof that $\mathrm{M}_{\phi(x ; p)}$ is continuous in $p$ for $p \in \mathbb{R} \backslash 0$ : The sum of continuous functions is continuous. For the cases of $p \in\{-\infty, 0, \infty\}$, see the items that follow.

(3) Lemma: $\mathrm{M}_{\phi(x ;-p)}\left(0 x_{n} D\right)=\left\{\mathrm{M}_{\phi(x ; p)}\left(0 x_{n}^{-1} D\right)\right\}^{-1}$. Proof:

$$
\begin{aligned}
\left\{\mathrm{M}_{\phi(x ; p)}\left(0 x_{n}^{-1} D\right)\right\}^{-1} & =\left\{\left(\sum_{n=1}^{N} \lambda_{n}\left(x_{n}^{-1}\right)^{p}\right)^{\frac{1}{p}}\right\}^{-1} \\
& =\left(\sum_{n=1}^{N} \lambda_{n}\left(x_{n}\right)^{-p}\right)^{\frac{1}{-p}} \\
& =\mathrm{M}_{\phi(x ;-p)}\left(0 x_{n} D\right)
\end{aligned}
$$

by definition of $\mathrm{M}_{\phi}$

by definition of $\mathrm{M}_{\phi}$

(4) Proof that $\lim _{p \rightarrow \infty} \mathrm{M}_{\phi}\left(0 x_{n} D\right)=\max _{n \in \mathbb{Z}}\left(x_{n} D\right.$ :

(a) Let $x_{m} \triangleq \max _{n \in \mathbb{Z}}\left(x_{n}\right)$

(b) Note that $\lim _{p \rightarrow \infty} \mathrm{M}_{\phi} \leq \max _{n \in \mathbb{Z}}\left(x_{n} D\right.$ because

$$
\begin{aligned}
\lim _{p \rightarrow \infty} \mathrm{M}_{\phi}\left(0 x_{n} D\right) & =\lim _{p \rightarrow \infty}\left(\sum_{n=1}^{N} \lambda_{n} x_{n}^{p}\right)^{\frac{1}{p}} & & \text { by definition of } \mathrm{M}_{\phi} \\
& \leq \lim _{p \rightarrow \infty}\left(\sum_{n=1}^{N} \lambda_{n} x_{m}^{p}\right)^{\frac{1}{p}} & & \begin{array}{l}
\text { by definition of } x_{m} \text { in item (4a) and because } \phi(x) \triangleq \\
x^{p} \text { and } \phi^{-1} \text { are both increasing or both decreasing }
\end{array} \\
& =\lim _{p \rightarrow \infty}(\underbrace{x_{n=1}^{p} \lambda_{n}^{N} \lambda_{n}^{\frac{1}{p}}}_{1} & & \text { because } x_{m} \text { is a constant }
\end{aligned}
$$




$$
\begin{array}{ll}
=\lim _{p \rightarrow \infty}\left(x_{m}^{p} \cdot 1\right)^{\frac{1}{p}} & \\
=x_{m} & \\
=\max _{n \in \mathbb{Z}}\left(x_{n}\right) & \text { by definition of } x_{m} \text { in item (4a) }
\end{array}
$$

(c) But also note that $\lim _{p \rightarrow \infty} \mathrm{M}_{\phi} \geq \max _{n \in \mathbb{Z}}\left(x_{n}\right)$ because

$$
\begin{aligned}
\lim _{p \rightarrow \infty} \mathrm{M}_{\phi}\left(0 x_{n} D\right) & =\lim _{p \rightarrow \infty}\left(\sum_{n=1}^{N} \lambda_{n} x_{n}^{p}\right)^{\frac{1}{p}} & & \text { by definition of } \mathrm{M}_{\phi} \\
& \geq \lim _{p \rightarrow \infty}\left(w_{m} x_{m}^{p}\right)^{\frac{1}{p}} & & \begin{array}{l}
\text { by definition of } x_{m} \text { in item (4a) and because } \phi(x) \triangleq \\
x^{p} \text { and } \phi^{-1} \text { are both increasing or both decreasing }
\end{array} \\
& =\lim _{p \rightarrow \infty} w_{m}^{\frac{1}{p}} x_{m}^{\frac{p}{p}} & & \\
& =x_{m} & & \text { by definition of } x_{m} \text { in item (4a) } \\
& =\max _{n \in \mathbb{Z}}\left(x_{n} D\right. & &
\end{aligned}
$$

(d) Combining items (b) and (c) we have $\lim _{p \rightarrow \infty} \mathrm{M}_{\phi}=\max _{n \in \mathbb{Z}}\left(x_{n} D\right.$.

(5) Proof that $\left.\lim _{p \rightarrow-\infty} \mathrm{M}_{\phi}\left(0 x_{n}\right)\right)=\min _{n \in \mathbb{Z}}\left(x_{n}\right)$ :

$$
\begin{array}{rlrl}
\lim _{p \rightarrow-\infty} \mathrm{M}_{\phi(x ; p)}\left(\left(x_{n} D\right)\right. & =\lim _{p \rightarrow \infty} \mathrm{M}_{\phi(x ;-p)}\left(0 x_{n} D\right) & & \text { by change of variable } p \\
& =\lim _{p \rightarrow \infty}\left\{\mathrm{M}_{\phi(x ; p)}\left(0 x_{n}^{-1} D\right)\right\}^{-1} & & \text { by Lemma in item (3) page 32 } \\
& =\lim _{p \rightarrow \infty} \frac{1}{\mathrm{M}_{\phi(x ; p)}\left(0 x_{n}^{-1} D\right)} & & \\
& =\frac{\lim _{p \rightarrow \infty} 1}{\lim _{p \rightarrow \infty} \mathrm{M}_{\phi(x ; p)}\left(0 x_{n}^{-1} D\right)} & & \text { by property of lim } 73 \\
& =\frac{1}{\max _{n \in \mathbb{Z}}\left(x_{n}^{-1} D\right.} & & \text { by item (4) } \\
& =\frac{1}{\left(\min _{n \in \mathbb{Z}}\left(x_{n} D\right)^{-1}\right.} & & \\
& =\min _{n \in \mathbb{Z}}\left(\mid x_{n} D\right. &
\end{array}
$$

(6) Proof that $\lim _{p \rightarrow 0} \mathrm{M}_{\phi}\left(0 x_{n} D\right)=\prod_{n=1}^{N} x_{n}^{\lambda_{n}}$ :

$$
\begin{aligned}
\lim _{p \rightarrow 0} \mathrm{M}_{\phi}\left(\left(0 x_{n} D\right)\right. & =\lim _{p \rightarrow 0} \exp \left\{\ln \left\{\mathrm{M}_{\phi}\left(0 x_{n} D\right)\right\}\right\} \\
& =\lim _{p \rightarrow 0} \exp \left\{\ln \left\{\left(\sum_{n=1}^{N} \lambda_{n}\left(x_{n}^{p}\right)\right)^{\frac{1}{p}}\right\}\right\} \quad \text { by definition of } \mathrm{M}_{\phi}
\end{aligned}
$$

\footnotetext{
${ }^{73} \boxminus[$ Rudin(1976)] page $85\langle 4.4$ Theorem $\rangle$
} 


$$
\begin{array}{ll}
=\exp \left\{\frac{\frac{\partial}{\partial p} \ln \left(\sum_{n=1}^{N} \lambda_{n}\left(x_{n}^{p}\right)\right)}{\frac{\partial}{\partial p} p}\right\}_{p=0} & \text { by l'Hôpital's rule }{ }^{74} \\
=\exp \left\{\frac{\sum_{n=1}^{N} \lambda_{n} \frac{\partial}{\partial p}\left(x_{n}^{p}\right)}{\sum_{n=1}^{N} \lambda_{n}\left(x_{n}^{p}\right)}\right\}_{p=0} & =\exp \left\{\frac{\sum_{n=1}^{N} \lambda_{n} \frac{\partial}{\partial p} \exp \left(\ln \left(x_{n}^{p}\right)\right)}{\sum_{n=1}^{N} \lambda_{n} \frac{\partial}{\partial p} \exp \left(r \ln \left(x_{n}\right)\right)}\right. \\
=\exp \left\{\lambda_{n}\right. & =\exp \left\{\sum_{n=1}^{N} \lambda_{n} \frac{\partial}{\partial p} \exp \left(p \ln \left(x_{n}\right)\right)\right\}_{p=0} \\
=\exp \left\{\sum_{n=1}^{N} \lambda_{n} \exp \left\{p \ln x_{n}\right\} \ln \left(x_{n}\right)\right\}_{p=0} & =\exp \left\{\sum_{n=1}^{N} \lambda_{n} \ln \left(x_{n}\right)\right\} \\
=\exp \left\{\sum_{n=1}^{N} \ln \left(x_{n}^{\lambda_{n}}\right)\right\} & =\exp \left\{\ln \prod_{n=1}^{N} x_{n}^{\lambda_{n}}\right\}=\prod_{n=1}^{N} x_{n}^{\lambda_{n}}
\end{array}
$$

Corollary B.8 ${ }^{75}$ Let $\left|x_{n}\right|_{1}^{N}$ be a tuple. Let $\mid \lambda_{n} D_{1}^{N}$ be a tuple of weighting values such that $\sum_{n=1}^{N} \lambda_{n}=1$.

$$
\min \left|x_{n}\right\rangle \leq \underbrace{\left(\sum_{n=1}^{N} \lambda_{n} \frac{1}{x_{n}}\right)^{-1}}_{\text {harmonic mean }} \leq \underbrace{\prod_{n=1}^{N} x_{n}^{\lambda_{n}}}_{\text {geometric mean }} \leq \underbrace{\sum_{n=1}^{N} \lambda_{n} x_{n}}_{\text {arithmetic mean }} \leq \max \mid x_{n} D
$$

QPROOF:

(1) These five means are all special cases of the power mean $\mathrm{M}_{\phi(x: p)}$ (Definition B.6 page 31):

$$
\begin{array}{lll}
p=\infty: & & \max \left(x_{n}\right) \\
p=1: & & \text { arithmetic mean } \\
p=0: & & \text { geometric mean } \\
p=-1: & & \text { harmonic mean } \\
p=-\infty: & & \min \left(x_{n}\right)
\end{array}
$$

(2) The inequalities follow directly from Theorem B.7 (page 31).

${ }^{74} \boxminus$ [Rudin(1976)] page $109\langle 5.13$ Theorem〉

$75 \otimes[$ [Bullen(2003)] page 71, $\boxminus$ [Bollobás(1999)] page 5, $\boxminus$ [Cauchy(1821)] pages 457-459 〈Note II, theorem 17〉, 圈 [Jensen(1906)] page 183, 圈 [Hoehn and Niven(1985)] page 151 
(3) Generalized AM-GM inequality: If one is only concerned with the arithmetic mean and geometric mean, their relationship can be established directly using Jensen's Inequality:

$$
\begin{array}{rlrl}
\sum_{n=1}^{N} \lambda_{n} x_{n} & =b^{\log _{b}\left(\sum_{n=1}^{N} \lambda_{n} x_{n}\right)} & \\
& \geq b^{\left(\sum_{n=1}^{N} \lambda_{n} \log _{b} x_{n}\right)} & \text { by Jensen's Inequality (Theorem B.2 page 30) } \\
& =\prod_{n=1}^{N} b^{\left(\lambda_{n} \log _{b} x_{n}\right)}=\prod_{n=1}^{N} b^{\left(\log _{b} x_{n}\right) \lambda_{n}}=\prod_{n=1}^{N} x_{n}^{\lambda_{n}}
\end{array}
$$

\section{B.3 Inequalities}

Lemma B.9 (Young's Inequality) 76

$$
\begin{aligned}
& x y<\frac{x^{p}}{p}+\frac{y^{q}}{q} \quad \text { with } \frac{1}{p}+\frac{1}{q}=1 \quad \forall 1<p<\infty, x, y \geq 0, \quad \text { but } y \neq x^{p-1} \\
& x y=\frac{x^{p}}{p}+\frac{y^{q}}{q} \quad \text { with } \frac{1}{p}+\frac{1}{q}=1 \quad \forall 1<p<\infty, x, y \geq 0, \quad \text { and } y=x^{p-1}
\end{aligned}
$$

Theorem B.10 (Minkowski's Inequality for sequences) ${ }^{77}$ Let $\| x_{n} \in \mathbb{C D}_{1}^{N}$ and $0 y_{n} \in \mathbb{C} D_{1}^{N}$ be complex $N$-tuples.

$$
\left(\sum_{n=1}^{N}\left|x_{n}+y_{n}\right|^{p}\right)^{\frac{1}{p}} \leq\left(\sum_{n=1}^{N}\left|x_{n}\right|^{p}\right)^{\frac{1}{p}}+\left(\sum_{n=1}^{N}\left|y_{n}\right|^{p}\right)^{\frac{1}{p}} \quad \forall 1<p<\infty
$$

\section{Appendix C Metric preserving functions}

Definition C.1 78 Let $\mathbb{M}$ be the set of all metric spaces (Definition 4.5 page 16) on a set $X . \quad \phi \in \mathbb{R}^{\vdash^{-\mathbb{R}^{+}}}$is a metric preserving function if $\quad \mathrm{d}(x, y) \triangleq \phi \circ \mathrm{p}(x, y) \quad$ is a metric on $X$ for all $(X, \mathrm{p}) \in \mathbb{M}$

Theorem C.2 (necessary conditions) ${ }^{79}$ Let $\mathcal{R} \phi$ be the RANGE of a function $\phi$.

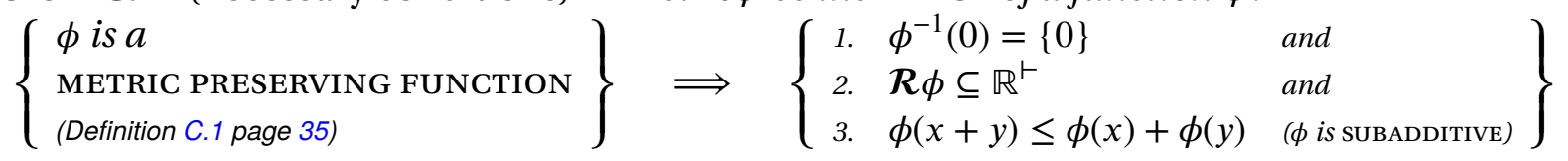

${ }^{76}$ 圈 [Young(1912)] page 226, $\bullet$ [Hardy et al.(1952)Hardy, Littlewood, and Pólya]〈Theorem 24〉, 圈 [Tolsted(1964)] page 5, 圈 [Maligranda(1995)] page 257, $\bullet$ [Carothers(2000)], page 43

$77 \boxminus$ [Minkowski(1910)], page 115, $\boxminus$ [Hardy et al.(1952)Hardy, Littlewood, and Pólya] $\langle$ Theorem 24〉, 圈 [Maligranda(1995)] page 258, $\bullet$ [Tolsted(1964)], page 7, $\bullet$ [Carothers(2000)], page 44, $₫$ [Bullen(2003)] page 179

$78 \boxminus$ [Vallin(1999)], page $849\langle$ Definition 1.1〉, $\boxminus$ [Corazza(1999)], page 309, $\boxminus$ [Deza and Deza(2009)] page 80

${ }_{79} \boxminus[$ Corazza(1999)], page $310\langle$ Proposition 2.1〉, $\boxminus$ [Deza and Deza(2009)] page 80 
Theorem C.3 (sufficient conditions) ${ }^{80}$ Let $\phi$ be a function in $\mathbb{R}^{\mathbb{R}}$.

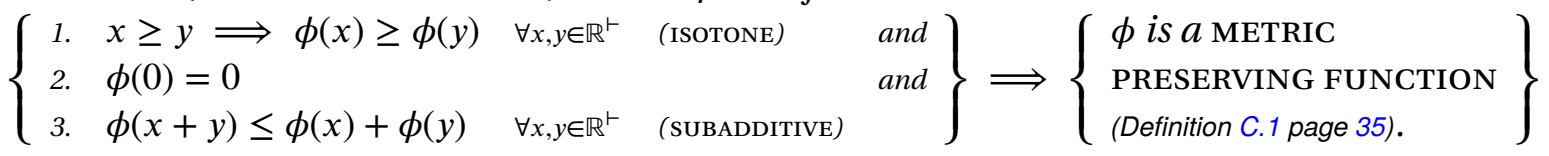
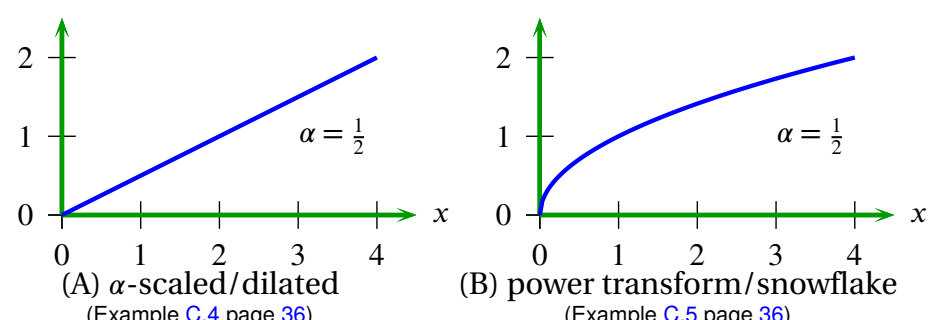

(B) power transform/snowflake
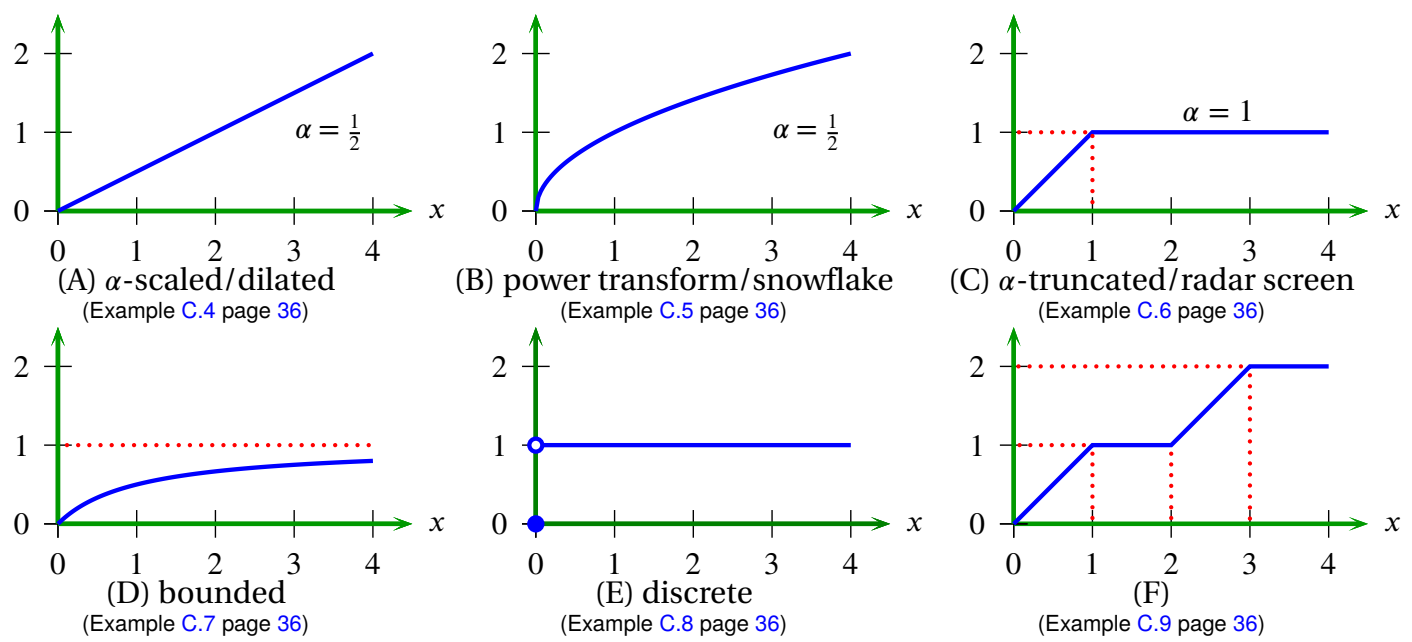

(C) $\alpha$-truncated/radar screen

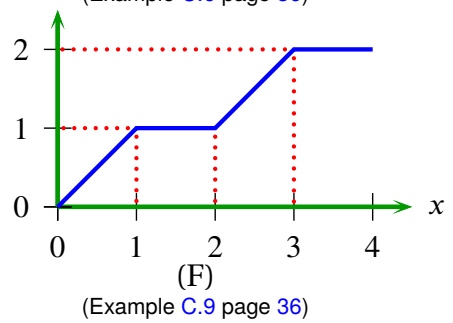

Figure 4: metric preserving functions

Example C.4 ( $\alpha$-scaled metric/dilated metric) ${ }^{81}$ Let $(X, d)$ be a metric space (Definition 4.5 page 16). $\phi(x) \triangleq \alpha x, \alpha \in \mathbb{R}^{+} \quad$ is a metric preserving function (Figure 4 page 36 (A))

$\otimes$ Proof: The proofs for Example C.4-Example C.9 (page 36) follow from Theorem C.3 (page 36).

Example C.5 (power transform metric/snowflake transform metric) ${ }^{82}$ Let $(X, \mathrm{~d})$ be a metric space (Definition 4.5 page 16). $\phi(x) \triangleq x^{\alpha}, \alpha \in(0: 1]$, is a metric preserving function (see Figure 4 page 36 (B))

Example C.6 ( $\alpha$-truncated metric/radar screen metric) 83 Let $(X, \mathrm{~d})$ be a metric space (Definition 4.5 page 16). $\phi(x) \triangleq \min \{\alpha, x\}, \alpha \in \mathbb{R}^{+} \quad$ is a metric preserving function (see Figure 4 page $36(\mathrm{C})$ ).

Example C.7 (bounded metric) ${ }^{84}$ Let $(X, \mathrm{~d})$ be a metric space (Definition 4.5 page 16). $\phi(x) \triangleq \frac{x}{1+x} \quad$ is a metric preserving function (see Figure 4 page 36 (D)).

Example C.8 (discrete metric preserving function) ${ }^{85}$ Let $\phi$ be a function in $\mathbb{R}^{\mathbb{R}}$. $\phi(x) \triangleq\left\{\begin{array}{ll}0 & \text { for } x \leq 0 \\ 1 & \text { otherwise }\end{array}\right\} \quad$ is a metric preserving function (see Figure 4 page $36(\mathrm{E})$ ).

Example C.9 Let $\phi$ be a function in $\mathbb{R}^{\mathbb{R}}$.

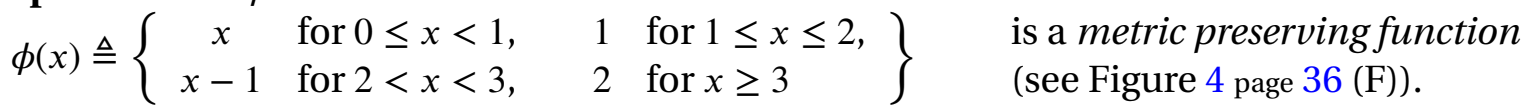

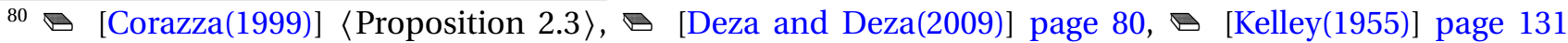
$\langle$ Problem C $\rangle$

${ }^{81} \boxminus[$ Deza and Deza(2006)] page 44

$82 \boxminus$ [Deza and Deza(2009)] page 81, $\theta$ [Deza and Deza(2006)] page 45

$83 \otimes$ [Giles(1987)], page 33, $\theta$ [Deza and Deza(2006)] pages 242-243

${ }^{84} \boxminus$ [Vallin(1999)], page 849, $\boxminus$ [Aliprantis and Burkinshaw(1998)] page 39

$85 \boxminus$ [Corazza(1999)], page 311 


\section{References}

[oei(2014)] , 2014. On-line encyclopedia of integer sequences. World Wide Web.

URL http: //oeis .org/

[Adams and Franzosa(2008)] Adams, C. C., Franzosa, R. D., 2008. Introduction to Topology: Pure and Applied. Featured Titles for Topology Series. Pearson Prentice Hall.

URL http: //books . google.com/books?vid=ISBN0131848690

[Aliprantis and Burkinshaw(1998)] Aliprantis, C. D., Burkinshaw, O., 1998. Principles of Real Analysis, 3rd Edition. Acedemic Press, London.

URL http: //www . amazon. com/dp/0120502577

[Apostol(1975)] Apostol, T. M., 1975. Mathematical Analysis, 2nd Edition. Addison-Wesley series in mathematics. Addison-Wesley, Reading.

URL http: //books . google. com/books?vid=ISBN0201002884

[Barvinok(2002)] Barvinok, A., 2002. A Course in Convexity. Vol. 54 of Graduate studies in mathematics. American Mathematical Society.

URL http://books . google.com/books?vid=ISBN0821872311

[Beran(1985)] Beran, L., 1985. Orthomodular Lattices: Algebraic Approach. Mathematics and Its Applications (East European Series). D. Reidel Publishing Company, Dordrecht.

URL http://books . google.com/books?vid=ISBN902771715X

[Berberian(1961)] Berberian, S. K., 1961. Introduction to Hilbert Space. Oxford University Press, New York. URL http: //books . google.com/books?vid=ISBN0821819127

[Bessenyei and Pales(2014)] Bessenyei, M., Pales, Z., January 8 2014. A contraction principle in semimetric spaces. arXiv.org.

URL http: //arxiv .org/abs/1401.1709

[Besso(1879)] Besso, D., 1879. Teoremi elementari sui massimi i minimi. Annuari Ist. Tech. Roma, 7-24See Bullen(2003) pages 453, 203.

[Bienaymé(1840)] Bienaymé, M. J., June 13 1840. Société philomatique de paris-extraits des procès-verbaux. Scéance, 67-68See Bullen(2003) pages 453, 203.

URL http://www . archive.org/details/extraitsdesproc46183941soci

[Blumenthal(1938)] Blumenthal, L. M., 1938. Distance geometries: a study of the development of abstract metrics. The University of Missouri studies. A quarterly of research 13 (2), 145.

[Blumenthal(1953)] Blumenthal, L. M., 1953. Theory and Applications of Distance Geometry, 1st Edition. Oxford at the Clarendon Press.

URL http: //books . google. com/books?vid=ISBN0828402426

[Bollobás(1999)] Bollobás, B., March 1 1999. Linear Analysis; an introductory course, 2nd Edition. Cambridge mathematical textbooks. Cambridge University Press, Cambridge.

URL http: //books . google.com/books?vid=ISBN0521655773

[Bottazzini(1986)] Bottazzini, U., 1986. The Higher Calculus: A History of Real and Complex Analysis from Euler to Weierstrass. Springer-Verlag, New York.

URL http://books . google.com/books?vid=ISBN0387963022

[Bourbaki(1939)] Bourbaki, 1939. Éléments de mathématique. Prewmieère partie: Les structures fondamentales de l'analyse. Livre I: Théorie des ensembles. Fascicule des résultats. Paris.

[Brenner(1985)] Brenner, J. L., Fall 1985. Limits of means for large values of the variable. Pi Mu Epsilon Journal 8 (3), 160-163.

URL http://www.pme-math.org/journal/issues .html 
[Brown and Watson(1996)] Brown, J. I., Watson, S., 15 June 1996. The number of complements of a topology on $\mathrm{n}$ points is at least $2^{n}$ (except for some special cases). Discrete Mathematics 154 (1-3), 27-39.

URL http://dx. doi .org/10.1016/0012-365X(95)00004-G

[Bruckner et al.(1997)Bruckner, Bruckner, and Thomson] Bruckner, A. M., Bruckner, J. B., Thomson, B. S., 1997. Real Analysis. Prentice-Hall, Upper Saddle River, N.J.

URL http: //books . google.com/books?vid=ISBN013458886X

[Brunschwig et al.(2003)Brunschwig, Lloyd, and Pellegrin] Brunschwig, J., Lloyd, G. E. R., Pellegrin, P., 2003. A Guide to Greek Thought: Major Figures and Trends. Harvard University Press.

URL http: //books . google.com/books?vid=ISBN0674021568

[Bryant(1985)] Bryant, V., 1985. Metric Spaces: Iteration and Application, illustrated, reprint Edition. Cambridge University Press, Cambridge.

URL http://books . google. com/books?vid=ISBN0521318971

[Bullen(1990)] Bullen, P. S., 1990. Averages still on the move. Mathematics Magazine 63 (4), 250-255.

URL http://www.maa.org/sites/default/files/Bullen94830627.pdf

[Bullen(2003)] Bullen, P. S., 2003. Handbook of Means and Their Inequalities, 2nd Edition. Vol. 560 of Mathematics and Its Applications. Kluwer Academic Publishers, Dordrecht, Boston.

URL http: //books . google.com/books?vid=ISBN1402015224

[Burstein et al.(2005)Burstein, Ulitsky, Tuller, and Chor] Burstein, D., Ulitsky, I., Tuller, T., Chor, B., May 14-18 2005. Information theoretic approaches to whole genome phylogenies. In: Miyano, S., Mesirov, J., Kasif, S., Istrail, S., Pevzner, P. A., Waterman, M. (Eds.), Research in Computational Molecular Biology. 9th Annual International Conference, RECOMB 2005. Vol. 3500 of Lecture Notes in Computer Science. Springer Science \& Business Media, pp. 283-295.

URL http://www.researchgate.net/profile/Tamir_Tuller/publication/221530206_Information_ Theoretic_Approaches_to_Whole_Genome_Phylogenies/links/0912f50b8c7a689215000000.pdf

[Carothers(2000)] Carothers, N., 2000. Real Analysis. Cambridge University Press, Cambridge. URL http: //books . google. com/books?vid=ISBN0521497566

[Cauchy(1821)] Cauchy, A.-L., 1821. Part 1: Analyse Algebrique. Cours D'Analyse de L'école Royale Polytechnique. Systems design course of the Polytechnic Royal School; 1st Part: Algebraic analysis).

URL http://www . archive.org/details/coursdanalysede00caucgoog

[Chatterji(1967)] Chatterji, S. D., July 1967. The Number of Topologies on $n$ Points. No. N67-31144. Techreport. URL http://ntrs.nasa.gov/archive/nasa/casi.ntrs.nasa.gov/19670021815_1967021815.pdf

[Choquet(1954)] Choquet, G., 1954. Theory of capacities. Annales de l'institut Fourier 5, 131-295. URL http: //aif . cedram.org/item?id=AIF_1954__5_-131_0

[Cohn(2002 December 6)] Cohn, P., 2002 December 6. Basic Algebra; Groups, Rings and Fields. Springer. URL http://books . google.com/books?vid=isbn1852335874

[Comtet(1966)] Comtet, L., 1966. Recouvrements, bases de filtre et topologies d'un ensemble fini. Comptes rendus de l'Acade'mie des sciences 262 (20), A1091-A1094, recoveries, bases and filter topologies of a finite set.

[Comtet(1974)] Comtet, L., 1974. Advanced combinatorics: the art of finite and infinite. D. Reidel Publishing Company, Dordrecht, translated and corrected version of the 1970 French edition.

URL http: //books . google. com/books?vid=ISBN9027704414

[Copson(1968)] Copson, E. T., 1968. Metric Spaces. No. 57 in Cambridge tracts in mathematics and mathematical physics. Cambridge University Press, London.

URL http: //books . google.com/books?vid=ISBN0521047226

[Corazza(1999)] Corazza, P., April 1999. Introduction to metric-preserving functions. The American Mathematical Monthly 104 (4), 309-323.

URL http://pcorazza.lisco.com/papers/metric-preserving.pdf 
[Costa et al.(2004)Costa, Castro, Rowstron, and Key] Costa, M., Castro, M., Rowstron, A., Key, P., 2004. Pic: Practical internet coordinates for distance estimation. In: Distributed Computing Systems, 2004. Proceedings. 24th International Conference on. pp. 178-187. URL http://research.microsoft.com/pubs/67616/tr-2003-53.pdf

[Crammer et al.(2007)Crammer, Kearns, and Wortman] Crammer, K., Kearns, M., Wortman, J., 2007. Learning from multiple sources. In: Scholkopf, B., Platt, J., Hofmann, T. (Eds.), Advances in Neural Information Processing Systems 19: Proceedings of the 2006 Conference. Vol. 19 of Neural information processing series. MIT Press, pp. 321-328.

URL http://machinelearning.wustl.edu/mlpapers/paper_files/NIPS2006_46.pdf

[Crossley(2006)] Crossley, M. D., 2006. Essential Topology. Springer Undergraduate Mathematics Series. Springer Science \& Business Media.

URL http://books . google. com/books?vid=ISBN1846281946

[Czerwik(1993)] Czerwik, S., 1993. Contraction mappings in b-metric spaces. Acta Mathematica et Informatica Universitatis Ostraviensis 1 (1), 5-11.

URL http://dml.cz/dmlcz/120469

[Davis(2005)] Davis, S. W., 2005. Topology. McGraw Hill, Boston.

URL http://www. worldcat.org/isbn/0071243399

[Dedekind(1900)] Dedekind, R., January 8 1900. Ueber die von drei moduln erzeugte dualgruppe. Mathematische Annalen 53, 371-403, regarding the Dual Group Generated by Three Modules.

URL http://resolver. sub.uni-goettingen.de/purl/?GDZPPN002257947

[Deza and Deza(2006)] Deza, E., Deza, M.-M., 2006. Dictionary of Distances. Elsevier Science, Amsterdam. URL http: //books . google.com/books?vid=ISBN0444520872

[Deza and Deza(2009)] Deza, M.-M., Deza, E., 2009. Encyclopedia of Distances. Springer.

URL http://www.uco.es/users/ma1fegan/Comunes/asignaturas/vision/ Encyclopedia-of-distances-2009.pdf

[Deza and Deza(2014)] Deza, M.-M., Deza, E., 2014. Encyclopedia of Distances, 3rd Edition. Springer, Bücher. URL http: //books . google. com/books?vid=ISBN3662443422

[DiBenedetto(2002)] DiBenedetto, E., 2002. Real Analysis. Birkhäuser Advanced Texts. Birkhäuser, Boston. URL http://books . google. com/books?vid=ISBN0817642315

[Dieudonné(1969)] Dieudonné, J. A., 1969. Foundations of Modern Analysis. Academic Press, New York. URL http://books . google. com/books?vid=ISBN1406727911

[Euclid(circa 300BC)] Euclid, circa 300BC. Elements. URL http://farside.ph.utexas.edu/euclid.html

[Evans et al.(1967)Evans, Harary, and Lynn] Evans, J., Harary, F., Lynn, M., 1967. On the computer enumeration of finite topologies. Communications of the ACM - Association for Computing Machinery 10, 295-297. URL http://portal. acm.org/citation. cfm?id=363282.363311

[Fagin et al.(2003a)Fagin, Kumar, and Sivakumar] Fagin, R., Kumar, R., Sivakumar, D., 2003a. Comparing top $k$ lists. In: In Proceedings of the ACM-SIAM Symposium on Discrete Algorithms. Society for Industrial and Applied Mathematics, pp. 28-36.

URL http://citeseerx.ist.psu.edu/viewdoc/summary?doi=10.1.1.119.6597

[Fagin et al.(2003b)Fagin, Kumar, and Sivakumar] Fagin, R., Kumar, R., Sivakumar, D., 2003b. Comparing top $k$ lists. SIAM Journal on Discrete Mathematics 17 (1), 134-160.

URL http://citeseerx. ist.psu.edu/viewdoc/download?doi=10.1.1.86.3234\&rep=rep1\&type=pdf

[Fréchet(1906)] Fréchet, M. R., 1906. Sur quelques points du calcul fonctionnel (on some points of functional calculation). Rendiconti del Circolo Matematico di Palermo 22, 1-74, rendiconti del Circolo Matematico di Palermo (Statements of the Mathematical Circle of Palermo). 
[Fréchet(1928)] Fréchet, M. R., 1928. Les Espaces abstraits et leur théorie considérée comme introduction a l'analyse générale. Borel series. Gauthier-Villars, Paris, abstract spaces and their theory regarded as an introduction to general analysis.

URL http: //books . google. com/books? id=9czoHQAACAAJ

[Galvin and Shore(1984)] Galvin, F, Shore, S. D., March 1984. Completeness in semimetric spaces. Pacific Journal Of Mathematics 113 (1), 67-75.

URL http://msp.org/pjm/1984/113-1/pjm-v113-n1-p04-s.pdf

[Gemignani(1972)] Gemignani, M. C., 1972. Elementary Topology, 2nd Edition. Addison-Wesley Series in Mathematics. Addison-Wesley Publishing Company, Reading, Massachusetts, a 1990 Dover "unabridged and corrected" edition has been published.

URL http: //books . google.com/books?vid=ISBN0486665224

[Gibbons et al.(1977)Gibbons, Olkin, and Sobel] Gibbons, J. D., Olkin, I., Sobel, M., 1977. Selecting and Ordering Populations: A New Statistical Methodology. John Wiley \& Sons, New York, a 1999 unabridged and corrected republication has been made available as an "SIAM Classics edition", ISBN 9781611971101. URL http://books . google. com/books?vid=ISBN1611971101

[Giles(1987)] Giles, J. R., 1987. Introduction to the Analysis of Metric Spaces. No. 3 in Australian Mathematical Society lecture series. Cambridge University Press, Cambridge.

URL http: //books . google.com/books?vid=ISBN0521359287

[Haaser and Sullivan(1991)] Haaser, N. B., Sullivan, J. A., 1991. Real Analysis. Dover Publications, New York. URL http: //books . google.com/books?vid=ISBN0486665097

[Hahn and Rosenthal(1948)] Hahn, H., Rosenthal, A., 1948. Set Functions. University of New Mexico Press. URL http: //books . google.com/books?vid=ISBN111422295X

[Halmos(1950)] Halmos, P. R., 1950. Measure Theory. The University series in higher mathematics. D. Van Nostrand Company, New York, 1976 reprint edition available from Springer with ISBN 9780387900889. URL http: //www . amazon. com/dp/0387900888

[Halmos(1960)] Halmos, P. R., 1960. Naive Set Theory. The University Series in Undergraduate Mathematics. D. Van Nostrand Company, Inc., Princeton, New Jersey.

URL http://books . google.com/books?vid=isbn0387900926

[Hardy et al.(1952)Hardy, Littlewood, and Pólya] Hardy, G. H., Littlewood, J. E., Pólya, G., 1952. Inequalities, 2nd Edition. Cambridge Mathematical Library. Cambridge University Press, Cambridge.

URL http: //books . google. com/books?vid=ISBN0521358809

[Hausdorff(1914)] Hausdorff, F., 1914. Grundzüge der Mengenlehre. Von Veit, Leipzig, properties of Set Theory. URL http: //books . google.com/books? id=KTs4AAAAMAAJ

[Hausdorff(1937)] Hausdorff, F., 1937. Set Theory, 3rd Edition. Chelsea Publishing Company, New York, 1957 translation of the 1937 German Grundzüge der Mengenlehre.

URL http://books . google.com/books?vid=ISBN0828401195

[Heath(1961)] Heath, R. W., 1961. A regular semi-metric space for which there is no semi-metric under which all spheres are open. Proceedings of the American Mathematical Society 12, 810-811.

URL http://www . ams .org/journals/proc/1961-012-05/S0002-9939-1961-0125562-9/

[Heinonen(2001)] Heinonen, J., January 1 2001. Lectures on Analysis on Metric Spaces. Universitext Series. Springer Science \& Business Media.

URL http://books . google. com/books?vid=ISBN0387951040

[Hoehn and Niven(1985)] Hoehn, L., Niven, I., 1985 1985. Mathematics Magazine 58 (3), 151-156.

URL http://www . jstor.org/stable/2689911

[Jensen(1906)] Jensen, J. L. W. V., December 1906. Sur les fonctions convexes et les ine'galite's entre les valeurs moyennes (on the convex functions and the inequalities between the average values). Acta Mathematica 30 (1), 175-193.

URL http://www.springerlink. com/content/r55q1411g840j446/ 
[Jiménez and Yukich(2006)] Jiménez, R., Yukich, J. E., 2006. Statistical distances based on Euclidean graphs. pp. 223-240.

URL http: //books . google.com/books?vid=ISBN0387233946

[Joshi(1983)] Joshi, K. D., 1983. Introduction To General Topology, 1st Edition. New Age International. URL http: //books . google.com/books?vid=ISBN0852264445

[Kelley(1955)] Kelley, J. L., 1955. General Topology. University Series in Higher Mathematics. Van Nostrand, New York, republished by Springer-Verlag, New York, 1975.

URL http: //books . google. com/books?vid=ISBN0387901256

[Khamsi and Kirk(2001)] Khamsi, M.A., Kirk, W., 2001. An Introduction to Metric Spaces and Fixed Point Theory. John Wiley, New York.

URL http://books . google. com/books?vid=isbn0471418250

[Kirk and Shahzad(2014)] Kirk, W., Shahzad, N., October 23 2014. Fixed Point Theory in Distance Spaces. SpringerLink: Bücher. Springer.

URL http://books . google.com/books?vid=isbn3319109278

[Korselt(1894)] Korselt, A., March 1894. Bemerkung zur algebra der logik. Mathematische Annalen 44 (1), $156-$ 157, referenced by Birkhoff(1948)p.133.

URL http: //www.springerlink. com/content/v681m56871273j73/

[Krishnamurthy(1966)] Krishnamurthy, V., February 1966. On the number of topologies on a finite set. The American Mathematical Monthly 73 (2), 154-157.

URL http://www.jstor.org/stable/2313548

[Kubrusly(2001)] Kubrusly, C. S., 2001. The Elements of Operator Theory, 1st Edition. Springer. URL http: //books . google. com/books?vid=ISBN0817641742

[Kubrusly(2011)] Kubrusly, C. S., 2011. The Elements of Operator Theory, 2nd Edition. Springer. URL http://books . google.com/books?vid=ISBN0817649972

[Laos(1998)] Laos, N. K., 1998. Topics in Mathematical Analysis and Differential Geometry. Vol. 24 of Series in pure mathematics. World Scientific.

URL http: //books . google. com/books?vid=ISBN9810231806

[Leathem(1905)] Leathem, J. G., 1905. Volume and surface integrals used in physics, 1st Edition. Vol. 1 of Cambridge Tracts in Mathematics and Mathematical Physics. Cambridge University Press.

URL http: //archive.org/details/volumesurfaceint01leatuoft

[Lin(1998)] Lin, D., 1998. An information-theoretic definition of similarity. In: Proceedings of the Internatioinal Conference on Machine Learning.

URL http://webdocs.cs. ualberta.ca/ lindek/papers/sim.pdf

[MacLane and Birkhoff(1999)] MacLane, S., Birkhoff, G., 1999. Algebra, 3rd Edition. AMS Chelsea Publishing, Providence.

URL http://books . google.com/books?vid=isbn0821816462

[Maddux(2006)] Maddux, R. D., July 27 2006. Relation Algebras, 1st Edition. Elsevier Science. URL http: //books . google.com/books?vid=ISBN0444520139

[Maligranda(1995)] Maligranda, L., March 1995. A simple proof of the hölder and the minkowski inequality. The American Mathematical Monthly 102 (3), 256-259.

URL http: //www. jstor.org/stable/2975013

[Maligranda and Orlicz(1987)] Maligranda, L., Orlicz, W., March 01 1987. On some properties of functions of generalized variation. Monatshefte für Mathematik 104 (1), 53-65.

URL http://gdz.sub.uni-goettingen.de/download/PPN362162050_0104/LOG_0008.pdf

[McCarty(1967)] McCarty, G., 1967. Topology: An Introduction With Application to Topological Groups. International Series in Pure and Applied Mathematics. McGraw-Hill Book Company, New York, 1988 Dover edition available.

URL http://www . amazon. com/dp/0486656330 
[Menger(1928)] Menger, K., 1928. Untersuchungen über allgemeine metrik. Mathematische Annalen 100, 75163, (Investigations on general metric).

URL http://link. springer. com/article/10.1007\%2FBF01455705

[Michel and Herget(1993)] Michel, A. N., Herget, C. J., 1993. Applied Algebra and Functional Analysis. Dover Publications, Inc., original version published by Prentice-Hall in 1981.

URL http: //books . google.com/books?vid=ISBN048667598X

[Milovanović and Milovanović(1979)] Milovanović, G. V., Milovanović, I., 1979. On a generalization of certain results of a. ostrowski and a. lupaş. University of Beograd. Publikacije Elektrotehničkog Fakulteta (634677), 62-69, ser. Mat. Fiz.

URL http://www.mi.sanu.ac.rs/ gvm/radovi/643.pdf

[Minkowski(1910)] Minkowski, H., 1910. Geometrie der Zahlen. Druck und Verlag von B.G. Teubner, Leipzig, geometry of Numbers.

URL http://www . archive .org/details/geometriederzahl00minkrich

[Mitrinović et al.(2010)Mitrinović, Pečarić, and Fink] Mitrinović, D. S., Pečarić, J. E., Fink, A. M., 2010. Classical and New Inequalities in Analysis. Vol. 61 of Mathematics and its Applications (East European Series). Kluwer Academic Publishers, Dordrecht, Boston, London.

URL http: //www . amazon. com/dp/0792320646

[Molchanov(2005)] Molchanov, I. S., 2005. Theory of Random Sets. Probability and Its Applications. Springer. URL http: //books . google.com/books?vid=ISBN185233892X

[Mulholland(1950)] Mulholland, H. P., 1950. On generalizations of minkowski's inequality in the form of a triangle inequality. Proceedings of the London Mathematical Society s2-51, 294-307, received 1946 October 10, read 1947 June 19.

URL http: //plms . oxfordjournals . org/content/s2-51/1/294. extract

[Munkres(2000)] Munkres, J. R., 2000. Topology, 2nd Edition. Prentice Hall, Upper Saddle River, NJ. URL http://www. amazon.com/dp/0131816292

[Murdeshwar(1990)] Murdeshwar, M. G., 1990. General Topology, 2nd Edition. New Age International. URL http://books . google. com/books?vid=isbn8122402461

[Ore(1935)] Ore, O., April 1935. On the foundation of abstract algebra. i. The Annals of Mathematics 36 (2), $406-$ 437.

URL http://www.jstor.org/stable/1968580

[Pap(1995)] Pap, E., 1995. Null-Additive Set Functions. Vol. 337 of Mathematics and Its Applications. Kluwer Academic Publishers.

URL http: //www . amazon. com/dp/0792336585

[Pečarić et al.(1992)Pečarić, Proschan, and Tong] Pečarić, J. E., Proschan, F., Tong, Y. L., 1992. Convex Functions, Partial Orderings, and Statistical Applications. Vol. 187 of Mathematics in Science and Engineering. Academic Press, San Diego, California.

URL http: //books . google.com/books?vid=ISBN0125492502

[Ponnusamy(2002)] Ponnusamy, S., 2002. Foundations of Functional Analysis. CRC Press. URL http://books . google.com/books?vid=ISBN0849317177

[Rana(2002)] Rana, I. K., 2002. An Introduction to Measure and Integration, 2nd Edition. Vol. 45 of Graduate Studies in Mathematics. American Mathematical Society, Providence, R.I.

URL http://books . google. com/books?vid=ISBN0821829742

[Riesz(1909)] Riesz, F., 1909. Stetigkeitsbegriff und abstrakte mengenlehre. In: Castelnuovo, G. (Ed.), Atti del IV Congresso Internazionale dei Matematici. Vol. II. Tipografia della R. Accademia dei Lincei, Rome, pp. 1824, 1908 April 6-11.

URL http://www.mathunion.org/ICM/ICM1908.2/Main/icm1908.2.0018.0024.ocr.pdf 
[Rosenlicht(1968)] Rosenlicht, M., 1968. Introduction to Analysis. Dover Publications, New York. URL http: //books . google.com/books?vid=ISBN0486650383

[Rudin(1976)] Rudin, W., 1976. Principles of Mathematical Analysis, 3rd Edition. McGraw-Hill, New York, library QA300.R8 1976.

URL http://books . google.com/books?vid=ISBN007054235X

[Salzmann et al.(2007)Salzmann, Grundhöfer, Hähl, and Löwen] Salzmann, H., Grundhöfer, T., Hähl, H., Löwen, R., 2007. The Classical Fields: Structural Features of the Real and Rational Numbers. Vol. 112 of Encyclopedia of Mathematics and its Applications. Cambridge University Press.

URL http: //books . google. com/books?vid=ISBN0521865166

[Schweizer and Sklar(1983)] Schweizer, B., Sklar, A., 1983. Probabilistic Metric Spaces. Elsevier Science Publishing Co., a 2005 Dover edition (ISBN 9780486143750) has been published which is "an unabridged republication of the work first published by Elsevier Science Publishing Co., Inc., in 1983.”.

URL http://books . google.com/books?vid=ISBN0486143759

[Searcóid(2006)] Searcóid, M. O., 2006. Metric Spaces. Springer Undergraduate Mathematics Series. Springer Science \& Business Media.

URL http://books . google. com/books?vid=ISBN1846286271

[Sherstnev(1962)] Sherstnev, A. N., 1962. Random normed spaces. questions of completeness. Kazan. Gos. Univ. Uchen. Zap. 122 (4), pages 3-20.

URL http://mi . mathnet.ru/uzku138

[Simon(2011)] Simon, B., May 19 2011. Convexity: An Analytic Viewpoint. Vol. 187 of Cambridge Tracts in Mathematics. Cambridge University Press.

URL http: //books . google.com/books?vid=ISBN1107007313

[Steen and Seebach(1978)] Steen, L. A., Seebach, J. A., 1978. Counterexamples in Topology, 2nd Edition. Springer-Verlag, a 1995 "unabridged and unaltered republication" Dover edition is available.

URL http://books . google. com. tw/books?vid=ISBN0486319296

[Suppes(1972)] Suppes, P., 1972. Axiomatic Set Theory. Dover Publications, New York. URL http://books . google. com/books?vid=ISBN0486616304

[Sutherland(1975)] Sutherland, W. A., 1975. Introduction to Metric and Topological Spaces, illustrated, reprint Edition. Oxford University Press.

URL http: //books . google. com/books?vid=ISBN0198531613

[Szirmai(2007)] Szirmai, J., 2007. The densest geodesic ball packing by a type of nil lattices. Contributions to Algebra and Geometry 48 (2), 383-397.

URL http://www .emis . ams .org/journals/BAG/vol.48/no.2/b48h2szi.pdf

[Thomson et al.(2008)Thomson, Bruckner, and Bruckner] Thomson, B. S., Bruckner, A. M., Bruckner, J. B., 2008. Elementary Real Analysis, 2nd Edition. www.classicalrealanalysis.com.

URL http://classicalrealanalysis.info/com/Elementary-Real-Analysis.php

[Thron(1966)] Thron, W. J., 1966. Topological structures. Holt, Rinehart and Winston, New York.

URL http: //books .google.com/books? id=JRM_AAAAIAAJ

[Tietze(1923)] Tietze, H. F. F., 1923. Beiträge zur allgemeinen topologie i. Mathematische Annalen 88 (3-4), 290 312.

URL http://link. springer.com/article/10.1007\%2FBF01579182

[Tolsted(1964)] Tolsted, E., 1964. An elementary derivation of cauchy, hölder, and minkowski inequalities from young's inequality. Mathematics Magazine 37, 2-12.

URL http://mathdl. maa. org/mathDL/22/?pa=content\&sa=viewDocument\&nodeId=3036

[Vallin(1999)] Vallin, R. W., 1999. Continuity and differentiability aspects of metric preserving functions. Real Analysis Exchange 25 (2), 849-868.

URL projecteuclid.org/euclid.rae/1230995419 
[Veltkamp(2001)] Veltkamp, R. C., May 2001. Shape matching: Similarity measures and algorithms. Shape Modeling and Applications, SMI 2001 International Conference on, 188-197.

URL http://dspace.library.uu.nl/bitstream/handle/1874/2540/2001-03.pdf

[Veltkamp and Hagedoorn(2000)] Veltkamp, R. C., Hagedoorn, M., November 2-4 2000. Shape similarity measures, properties and constructions. In: Laurini, R. (Ed.), Advances in Visual Information Systems 4th International Conference, VISUAL 2000 Lyon. Vol. 1929 of Lecture Notes in Computer Science. Springer, France, pp. 467-476.

URL http://link. springer.com/chapter/10.1007/3-540-40053-2_41

[Vitányi(2011)] Vitányi, P. M. B., April 2011. Information distance in multiples. IEEE Transactions on Information Theory 57 (4).

URL http://arxiv.org/pdf/0905.3347.pdf

[Walmsley(1920)] Walmsley, C., 1920. An Introductory Course Of Mathematical Analysis. Cambridge University Press.

URL https://archive.org/details/introductorycour032788mbp

[Wilson(1931)] Wilson, W. A., April 1931. On semi-metric spaces. American Journal of Mathematics 53 (2), 361373.

URL http://www.jstor.org/stable/2370790

[Xia(2009)] Xia, Q., April 2009. The geodesic problem in quasimetric spaces. Journal of Geometric Analysis 19 (2), 452-479.

URL http://link. springer. com/article/10.1007/s12220-008-9065-4

[Young(1912)] Young, W. H., August 1912. On classes of summable functions and their fourier series. Proceedings of the Royal Society of London 87 (594), 225-229.

URL http: //www . archive .org/details/philtrans02496252

[Zorich(2004)] Zorich, V. A., 2004. Mathematical Analysis I. Universitext Series. Springer Science \& Business Media.

URL http://books . google. com/books?vid=ISBN3540403868

\section{Reference Index}

[Aliprantis and Burkinshaw(1998)], 6, 19, 21, 27, 36

[Adams and Franzosa(2008)], 8

[Apostol(1975)], 5, 9, 21

[Barvinok(2002)], 30

[Beran(1985)], 5

[Berberian(1961)], 18, 23

[Bessenyei and Pales(2014)], 6, 16

[Besso(1879)], 31

[Bienaymé(1840)], 31

[Blumenthal(1938)], 6

[Blumenthal(1953)], 6, 11-15

[Bollobás(1999)], 27, 30, 31, 34

[Bottazzini(1986)], 4

[Bourbaki(1939)], 4

[Brenner(1985)], 31

[Brown and Watson(1996)], 8
[Bruckner et al.(1997)Bruckner,

Bruckner, and Thomson], 6

[Brunschwig et al.(2003)Brunschwig,

Lloyd, and Pellegrin], 15

[Bryant(1985)], 10

[Bullen(1990)], 31

[Bullen(2003)], 31, 34, 35

[Burstein et al.(2005)Burstein,

Ulitsky, Tuller, and Chor], 2

[Carothers(2000)], 31, 35

[Cauchy(1821)], 34

[Chatterji(1967)], 8

[Choquet(1954)], 5

[Cohn(2002 December 6)], 5

[Comtet(1966)], 8

[Comtet(1974)], 4, 8

[Copson(1968)], 6, 16

[Corazza(1999)], 35, 36
[Costa et al.(2004)Costa, Castro, Rowstron, and Key], 2 [Crammer et al.(2007)Crammer, Kearns, and Wortman], 2 [Crossley(2006)], 28

[Czerwik(1993)], 16

[Davis(2005)], 11, 27, 28

[Dedekind(1900)], 5

[Deza and Deza(2006)], 36

[Deza and Deza(2009)], 35, 36

[Deza and Deza(2014)], 6, 16

[DiBenedetto(2002)], 26

[Dieudonné(1969)], 7, 16, 18

[Euclid(circa 300BC)], 16

[Evans et al.(1967)Evans, Harary, and Lynn], 8

[Fagin et al.(2003a)Fagin, Kumar, and Sivakumar], 16 
[Fagin et al.(2003b)Fagin, Kumar, and Sivakumar], 16

[Fréchet(1906)], 16

[Fréchet(1928)], 16

[Galvin and Shore(1984)], 6

[Gemignani(1972)], 27

[Gibbons et al.(1977)Gibbons,

Olkin, and Sobel], 16

[Giles(1987)], 9, 21, 36

[Haaser and Sullivan(1991)], 10,29

[Hahn and Rosenthal(1948)], 4,5

[Halmos(1950)], 5

[Halmos(1960)], 4

[Hardy et al.(1952)Hardy, Littlewood, and Pólya], 31, 35

[Hausdorff(1914)], 26

[Hausdorff(1937)], 6, 11, 16, 26

[Heath(1961)], 6

[Heinonen(2001)], 16

[Hoehn and Niven(1985)], 16, 34

[Jensen(1906)], 30, 34

[Jiménez and Yukich(2006)], 2

[Joshi(1983)], 8, 27, 28

[Kelley(1955)], 4, 27, 36

[Khamsi and Kirk(2001)], 6, 9

[Kirk and Shahzad(2014)], 16

[Korselt(1894)], 5

[Krishnamurthy(1966)], 8
[Kubrusly(2001)], 10, 27, 29

[Kubrusly(2011)], 26

[Laos(1998)], 6, 15

[Leathem(1905)], 9, 28

[Lin(1998)], 2

[MacLane and Birkhoff(1999)], 5

[Maddux(2006)], 4

[Maligranda and Orlicz(1987)],

2

[Maligranda(1995)], 31, 35

[McCarty(1967)], 27, 28

[Menger(1928)], 6

[Michel and Herget(1993)], 6, 18

[Milovanović and Milo-

vanović(1979)], 31

[Minkowski(1910)], 31, 35

[Mitrinović et al.(2010)Mitrinović,

Pečarić, and Fink], 30

[Molchanov(2005)], 4, 6

[Mulholland(1950)], 31

[Munkres(2000)], 26, 27, 29

[Murdeshwar(1990)], 8

[Ore(1935)], 5

[Pap(1995)], 4, 5

[Pečarić et al.(1992)Pečarić,

Proschan, and Tong], 31

[Ponnusamy(2002)], 28, 29

[Rana(2002)], 4

[Riesz(1909)], 26
[Rosenlicht(1968)], 7, 9, 10, 2123, 29

[Rudin(1976)], 33, 34

[Salzmann et al.(2007)Salzmann,

Grundhöfer, Hähl, and

Löwen], 8

[Schweizer and Sklar(1983)], 16

[Searcóid(2006)], 28

[Sherstnev(1962)], 16

[Simon(2011)], 30

[Steen and Seebach(1978)], 26

[Suppes(1972)], 4

[Sutherland(1975)], 10

[Szirmai(2007)], 2

[Thomson et al.(2008)Thomson,

Bruckner, and Bruckner], 23

[Thron(1966)], 6, 27

[Tietze(1923)], 26

[Tolsted(1964)], 31, 35

[Vallin(1999)], 35, 36

[Veltkamp and Hagedoorn(2000)], 2

[Veltkamp(2001)], 2

[Vitányi(2011)], 2

[Walmsley(1920)], 15

[Wilson(1931)], 6

[Xia(2009)], 16

[Young(1912)], 35

[Zorich(2004)], 19

\section{Subject Index}

\author{
$\alpha$-scaled, 36 \\ $\alpha$-scaled metric, 36 \\ $\alpha$-truncated, 36 \\ $\alpha$-truncated metric, 36 \\ $\phi$-mean, 31 \\ $\sigma$-inframetric inequality, 3,16 , \\ 16 \\ $\sigma$-inframetric space, 3,16 \\ absolute value, 5, 5, 12, 18 \\ accumulation point, 3, 4, 27 \\ adherent, 27 \\ affine, 17, 30 \\ AM-GM inequality, 34 \\ anti-symmetric, 5 \\ arithmetic mean, 1, 3, 17 \\ arithmetic mean geometric \\ mean inequality, 34 \\ b-metric, 16
}

base, 3, 4, 6, 8, 9, 12-14, 20, 27, 27

boundary, 27

bounded, 2, 6, 9, 10, 21, 22, 36

bounded metric, 36

Cantor Intersection Theorem, 2

Cantor intersection theorem,

11,11

cardinality, 5

cardinality of $A, 5$

Cartesian product, 4

Cauchy, 2, 3, 6, 9, 10, 13, 14, 21, 22, 25

Cauchy condition, 9

Cauchy sequence, $\mathbf{9}$

Cauchy sequences, 9

closed, 7, 7, 10, 11, 26, 27, 29,
30

closed ball, $\mathbf{6}$

closed interval, $\mathbf{5}$

closed set, $3,4,7$

closed set theorem, 30

closure, 3, 4, 10, 27, 27, 29

closure point, 27

commutative, 18, 23

commutative ring, 5

complete, 9, 10, 11

concave, 30, 30, 31, 32

continuity, $3,4,12,15,17,28-$

30

continuous, $1,3,6,11,11,12$,

$15,17,23,25,26,28,28,29-32$

contradiction, 24

converge, $\mathbf{9}$

convergence, $3,4,13,22,30$

metric space, 21 
convergent, 3, 6, 9, 9, 10-14, 21, 22, 25, 29, 30

convergent sequence, 3

converges, 28, 29

convex, 30, 30, 31, 32

functional, 30

strictly, 30

decreasing, 31

definitions

$\sigma$-inframetric space, $\mathbf{1 6}$

accumulation point, 27

base, 27

Cartesian product, $\mathbf{4}$

Cauchy sequence, $\mathbf{9}$

closed ball, 6

closed interval, $\mathbf{5}$

closed set, 7

closure point, 27

distance space, $\mathbf{6}$

empty set, $\mathbf{5}$

extended real numbers, 4

function, 4

half-open interval, $\mathbf{5}$

inframetric space, 16

interior, 27

interior point, $\mathbf{2 7}$

metric space, 16

near metric space, 16

open ball, 6

open interval, $\mathbf{5}$

open set, 7

ordered pair, 4

ordered set, $\mathbf{5}$

partially ordered set, $\mathbf{5}$

paving, 4

point of adherence, 27

poset, $\mathbf{5}$

power distance space, 16

relation, 4

set function, $\mathbf{5}$

set of all functions, 4

set of all relations, 4

set of integers, 4

set of natural numbers, 4

set of non-negative real

numbers, 4

bers, 4

set of postive real num-

set of real numbers, 4

set structure, 4

topological space in-

duced by $(X, \mathrm{~d}), \mathbf{8}$

topology, $\mathbf{2 6}$

topology induced by

$(X, \mathrm{~d})$ on $X, \mathbf{8}$

diameter, 6 dilated, 36

dilated Euclidean, 14, 25

dilated metric, 36

discontinuous, 11, 12-15, 28,

28

discrete, 13, 24, 36

discrete metric preserving

function, 36

discrete topology, 26, 28

distance, 6, 6, 12-15

distance function, $11,12,14,15$

distance space, 1-6, 6, 7-17, 21,

23-25

distance spaces, 3

diverge, 14, 29

divergent, 29

empty set, 5, 7

Euclidean, 12-14, 24, 25

Euclidean metric space, 25

examples

$\alpha$-scaled, 36

$\alpha$-scaled metric, 36

$\alpha$-truncated, 36

$\alpha$-truncated metric, 36

bounded, 36

bounded metric, 36

dilated, 36

dilated metric, 36

discrete, 36

discrete metric preserving

function, 36

power transform, 36

power transform metric,

36

radar screen, $\mathbf{3 6}$

radar screen metric, $\mathbf{3 6}$

snowflake, 36

snowflake transform met-

ric, 36

the standard topology on

the real line, $\mathbf{2 7}$

extended real numbers, 4, 15

extended set of integers, 4

finite, 5

function, 4, 28

functions

absolute value, 5, 5, 12, 18

arithmetic mean, 17

cardinality of $A, 5$

distance, 6, 6, 12-15

15

distance function, 11, 14,

geometric mean, 17

harmonic mean, 17

maximum, 17 metric, 14

metric function, 3,12

metric preserving func-

tion, 35, 35, 36

minimum, 17

modulus, $\mathbf{5}$

power distance, 16

power distance function,

16, 17, 26

Power mean, 3, 15

power mean, $1,3,15,31$,

31

power triangle function,

$1,3,15,15,16,17,22,23$

quadratic mean, 17

sequence, $6,9-11$

set function, 5

triangle function, 16, 25

Generalized AM-GM inequality, 35

generalized arithmetic mean geometric mean inequality, 34 geometric inequality, 16, 18 geometric mean, $1,3,17$

half-open interval, 5, 12

harmonic inequality, 16, 18

harmonic mean, $1,3,17$

homogeneous, 5

horizontal half-open interval, 12,24

increasing, 31

indiscrete topological space, 8 indiscrete topology, 26, 28

inequalities

AM-GM, 34

Jensen's, 30

35

Minkowski (sequences),

23

power triangle inequality,

Young, 35

inframetric inequality, 1,16 , 16, 18

inframetric space, 16, 17

interior, 3, 4, 27, 27

interior point, 27

inverse image characteriza-

tion, 12

inverse image characterization

of continuity, 12, 29

isotone, 36

Jensen's Inequality, 30, 35

l'Hôpital's rule, 34 
limit, 9, 10, 29, 29

limit point, 29

linearly ordered, 8

maximum, 1, 3, 17

mean square, 1,3

metric, 14, 35

metric function, 3,12

metric preserving function, 35,

35, 36

Metric space, 1, 2

metric space, 2-16, 16, 18, 21$25,27-29,35,36$

metrics

$\alpha$-scaled metric, 36

$\alpha$-truncated metric, 36

bounded, 36

dilated metric, 36

power transform metric,

36

radar screen, 36

minimal inequality, 16, 18

minimum, 1, 3, 17

Minkowski's Inequality, 31

Minkowski's Inequality for se-

quences, 35

modulus, $\mathbf{5}$

monontone, 3

monotone, 1,19

near metric space, $3,16, \mathbf{1 6}, 17$

non-negative, $5,6,18,24,31$

nondegenerate, 5, 6, 19, 24

not Cauchy, 14

not closed, 30

not open, 12,13

not unique, 13, 23, 29

open, $2-4,6,7,7,8,9,12-14$, 20, 21, 26, 26, 27-29

open ball, 3, 4, 6, 6, 7-9, 12-14, 19-21

open balls, 12,20

open interval, 5, 12

open set, $7,7,8,12,13,20,27-$

29

operations

$\phi$-mean, 31

arithmetic mean, 1, 3

closure, 27

geometric mean, 1, 3

harmonic mean, 1, 3

limit, 9, 29

maximum, 1,3

mean square, 1, 3

minimum, 1, 3

open set, 7 set difference, 7

set intersection, 7

SMR, 16

square-mean-root, 16

order relation, $5, \mathbf{5}$

ordered metric space, 16

ordered pair, 4, 12

ordered set, 5,5

ordered set of real numbers, 5

partial order relation, $\mathbf{5}$

partially ordered set, $\mathbf{5}$

paving, 4, 5

point of adherence, $\mathbf{2 7}$

poset, $\mathbf{5}$

positive real numbers, 15

power distance, 16

power distance function, $\mathbf{1 6}$

17,26

power distance space, $3,4, \mathbf{1 6}$,

$17,19-25$

power distance spaces, 3

Power mean, 3, 15

power mean, 1, 3, 15, 31, 31, 34

power set, 4, 6, 26, 27

power set of $X, 4$

power transform, 36

power transform metric, 36

power triangle function, 1,3 , $15,15,16,17,22,23$

power triangle inequality, 1,3 , $15,16,16,17,18,21-23,25,26$

power triangle triangle space, 18

preorder, 5

probabilistic metric spaces, 16

properties

adherent, 27

affine, 17, 30

anti-symmetric, 5

bounded, 2, 6, 9, 10, 21, 22

Cauchy, 2, 3, 6, 9, 10, 13,

$14,21,22,25$

Cauchy condition, 9

closed, 7, 7, 10, 11, 26, 27, 29,30

closed set, 4

commutative, 18, 23

complete, 9, 10, 11

concave, 30, 30, 31, 32 28-30

continuity, $3,4,12,15,17$,

continuous, $1,3,6,11, \mathbf{1 1}$,

$12,15,17,23,25,26,28,28,29$

32

converge, 9

convergence, $3,4,13,22$,
30

convergent, $3,6,9,9,10$

$14,21,22,25, \mathbf{2 9}, 30$

converges, 28, 29

convex, 30, 30, 31, 32

decreasing, 31

dilated Euclidean, 14, 25

discontinuous, 11, 12-15,

28, 28

discrete, 13, 24

distance space, 11

diverge, 14, 29

divergent, 29

Euclidean, 12-14, 24, 25

finite, 5

homogeneous, 5

increasing, 31

isotone, 36

limit, $\mathbf{9}$

linearly ordered, 8

monontone, 3

monotone, 1,19

non-negative, $5,6,18,24$,

31

nondegenerate, 5, 6, 19,

24

not Cauchy, 14

not closed, 30

not open, 12,13

not unique, 13, 23, 29

open, $2,3,6,7,7,8,9,12-$

14, 20, 21, 26, 26, 27, 28

open set, 8

power triangle inequality,

$17,18,26$

reflexive, 5

strictly convex, $\mathbf{3 0}$

strictly monontone, 15

strictly monotone, 10,17 ,

$18,22,25,31,32$

strictly monotonic, 31

subadditive, 5, 35, 36

submultiplicative, 5

symmetric, 6, 18, 19, 22

transitive, 5

triangle inequality, $2,3,5$, 12-15, 23, 25

unique, $3,6,10,12-14,25$,

29

quadratic inequality, 16

quadratic mean, 17

quadratic mean inequality, 18

radar screen, $\mathbf{3 6}$

radar screen metric, $\mathbf{3 6}$

range, 35 
real line, 8

reflexive, 5

relation, 4, 4, 16

relations

$\sigma$-inframetric inequality,

$3,16,16$

geometric inequality, 16 harmonic inequality, 16

16,16

inframetric inequality, 1 ,

minimal inequality, 16

order relation, $\mathbf{5}$

partial order relation, $\mathbf{5}$

power triangle inequality,

$1,3,15,16,16,21,22,25$

preorder, 5

quadratic inequality, 16

relaxed triangle inequal-

ity, 1, 3, 16, 16

square mean root in-

equality, 16

triangle inequality, 1,2 ,

$15,16,16$

triangle relation, 24,25

relaxed triangle inequality, 1,3 , 16,16

sequence, 6, 9-11

sequences

Cauchy, 9

sequential characterization, 12 sequential characterization of continuity, 12, 29

set, 5,7

set difference, 7

set function, 5, $\mathbf{5}$

set intersection, 7

set of all functions, 4

set of all relations, 4

set of extended real numbers, 3

set of integers, 4

set of natural numbers, 4

set of non-negative real num-

bers, 4

set of positive real numbers, 3 , 6

set of postive real numbers, 4

set of real numbers, 4

set structure, 4

sets, 4

SMR, 16

snowflake, 36

snowflake transform metric, 36

space

metric, 7

topological, 26

square mean root (SMR) in- equality, 18

square mean root inequality,

16

square-mean-root, 16

strictly convex, 30, $\mathbf{3 0}$

strictly monontone, 15

strictly monotone, $10,17,18$,

22, 25, 31, 32

strictly monotonic, 31

structures

$\sigma$-inframetric space, $3, \mathbf{1 6}$

accumulation point, 3 , 4 ,

27

b-metric, 16

base, $3,4,6,8,9,12-14$,

$20,27,27$

boundary, 27

Cartesian product, 4

Cauchy sequence, $\mathbf{9}$

closed ball, 6

closed interval, $\mathbf{5}$

closed set, 3, 7

closure, 3, 4, 10, 27, 29

closure point, $\mathbf{2 7}$

commutative ring, 5

convergent sequence, 3

discrete topology, 26, 28

distance function, 12

distance space, 1-6, 6, 7-

17, 21, 23-25

distance spaces, 3

empty set, 5, 7

Euclidean metric space,

25

extended real numbers, 4

15

extended set of integers, 4

function, 4, 28

geometric inequality, 18

half-open interval, 5, 12

harmonic inequality, 18

horizontal half-open in-

terval, 12,24

indiscrete topological

space, 8

indiscrete topology, 26, 28

inframetric inequality, 18

inframetric space, 16, 17

interior, 3, 4, 27, 27

interior point, 27

limit, 29

metric, 35

Metric space, 1, 2

metric space, 2-16, 16, 18,

21-25, 27-29, 35, 36

minimal inequality, 18 near metric space, 3,16 ,

16, 17

open, 4,29

open ball, $3,4,6,6,7-9$,

12-14, 19-21

open balls, 12, 20

open interval, 5, 12

27-29

open set, $7,7,12,13,20$,

order relation, 5

ordered metric space, 16

ordered pair, 4, 12

ordered set, $5, \mathbf{5}$

ordered set of real num-

bers, 5

partially ordered set, $\mathbf{5}$

paving, 4, 5

point of adherence, 27

poset, 5

positive real numbers, 15

power distance space, 3 ,

$4,16,17,19-25$

power distance spaces, 3

power set, 4, 6, 26, 27

power set of $X, 4$

power triangle inequality,

3

power triangle triangle

space, 18 ity, 18

quadratic mean inequal-

range, 35

real line, 8

relation, 4, 4, 16

set, 5,7

set function, $\mathbf{5}$

set of all functions, 4

set of all relations, 4

set of extended real num-

bers, 3

set of integers, 4

set of natural numbers, 4

set of non-negative real

numbers, 4

set of positive real num-

bers, 3,6

set of postive real num-

bers, 4

set of real numbers, 4

set structure, 4

sets, 4

square mean root (SMR)

inequality, 18

topological space, $3,4,8$,

$12,23,26-29$

topological space $(X, T)$ 
induced by $(\mathbb{R}, d), 13$ topological space induced by $(\mathbb{R}, d), 12$ topological space induced by $(X, \mathrm{~d}), 8, \mathbf{8}, 10,21$ topologogical space, 8 topology, 3, 4, 8, 12-14, 26, 26 topology induced by

$(X, \mathrm{~d})$ on $X, \mathbf{8}$

triangle inequality, 2, 18

triangle relation, 16

trivial topology, 26

usual metric space, 27 vertical half-open inter-

val, 12, 24

weights, 30

subadditive, 5, 35, 36

submultiplicative, 5

symmetric, 6, 18, 19, 22

The Archimedean Property, 19

The Closed Set Theorem, 29

The principle of Archimedes, 19

the standard topology on the real line, 27

theorems

Cantor intersection, 11

Cantor Intersection Theorem, 2

Cantor intersection theorem, 11 closed set theorem, 30

Generalized AM-GM inequality, 35

inverse image characteri-

zation, 12

inverse image characteri-

zation of continuity, 12, 29

Jensen's Inequality, 30, 35

l'Hôpital's rule, 34

Minkowski's Inequality,

31

Minkowski's Inequality

for sequences, $\mathbf{3 5}$

sequential characteriza-

tion, 12

sequential characteriza-

tion of continuity, 12, 29 erty, 19

The Archimedean Prop-

The Closed Set Theorem,

29

The principle of

Archimedes, 19

Uniqueness of limit, 23

Young's Inequality, 35

topological space, $3,4,8,12$, 23, 26-29

topological space $(X, T)$ induced by $(\mathbb{R}, d), 13$

topological space induced by $(\mathbb{R}, d), 12$

topological space induced by
$(X, \mathrm{~d}), 8, \mathbf{8}, 10,21$

topologies

discrete, 26

indiscrete, 26

trivial, 26

topologogical space, 8

topology, 3, 4, 8, 12-14, 26, 26

topology induced by $(X, \mathrm{~d})$ on

$X, 8$

transitive, 5

triangle function, 16, 25

triangle inequality, $1-3,5,12-$

$16,16,18,23,25$

triangle relation, $16,24,25$

trivial topology, 26

unique, 3, 6, 10, 12-14, 25, 29

Uniqueness of limit, 23

usual metric space, 27

values

cardinality, 5

contradiction, 24

diameter, 6

limit, 10

limit point, 29

vertical half-open interval, 12 , 24

weighted, 31

weights, 30

Young's Inequality, 35

Telecommunications Engineering Department, National Chiao-Tung University, Hsinchu, Taiwan; 國立交通大學 (Gúo Lì Jiāo Tōng Dà Xúe) 電信工程學系 (Diàn Xìn Gōng Chéng Xúe Xì) 新竹，台灣 (Xīn Zhú, Tái Wān)

dgreenhoe@gmail.com

Received: aa bb 20YY Revised: cc dd 20ZZ 